J. DIFFERENTIAL GEOMETRY

85 (2010) 271-314

\title{
ON THE ALMOST SURE SPIRALING OF GEODESICS IN NEGATIVELY CURVED MANIFOLDS
}

\author{
SA’ar Hersonsky \& Frédéric Paulin
}

\begin{abstract}
Given a negatively curved geodesic metric space $M$, we study the almost sure asymptotic penetration behavior of (locally) geodesic lines of $M$ into small neighborhoods of points, of closed geodesics, and of other compact (locally) convex subsets of $M$. We prove Khintchine-type and logarithm law-type results for the spiraling of geodesic lines around these objets. As a consequence in the tree setting, we obtain Diophantine approximation results of elements of non-archimedian local fields by quadratic irrational ones.
\end{abstract}

\section{Introduction}

Let $M$ be a compact connected Riemannian manifold with negative sectional curvature. Endow the total space of the unit tangent bundle $\pi: T^{1} M \rightarrow M$ with the Bowen-Margulis measure $\mu$, which is the maximal entropy probability measure for the geodesic flow $\left(\phi_{t}\right)_{t \in \mathbb{R}}$ on $T^{1} M$. Let $h$ be the topological entropy of $\left(\phi_{t}\right)_{t \in \mathbb{R}}$. In this paper, we study the almost sure asymptotic penetration behavior of (locally) geodesic lines into various objets in $M$, as tubular neighborhoods of closed geodesic, tubular neighborhoods of compact embedded totally geodesic submanifolds, and other convex subsets. In this introduction, we fix a Lipschitz $\operatorname{map} g: \mathbb{R}_{+} \rightarrow \mathbb{R}_{+}$.

We first consider a closed geodesic $C$ in $M$, and study the spiraling of geodesics lines around $C$. As the geodesic flow is ergodic with respect to $\mu$, almost every orbit in $T^{1} M$ is dense. Two geodesic lines, having at some time their unit tangent vectors close, follow themselves closely a long time. Hence almost every geodesic line will stay for arbitrarily long periods of times in a given small neighborhood of $C$. In this paper, we make this behaviour quantitative. For that, we prove a Khintchine-type theorem, and a logarithm law-type corollary, for geodesic lines spiraling around $C$. Fix a small enough $\epsilon>0$, and let $\mathscr{N}_{\epsilon} C$ be the (closed) $\epsilon$-neighborhood of $C$.

Received 09/30/2007. 
Theorem 1.1. If $\int_{1}^{+\infty} e^{-h g(t)} d t$ converges (resp. diverges), then for $\mu$-almost no (resp. every) $v \in T^{1} M$, there exist positive times $\left(t_{n}\right)_{n \in \mathbb{N}}$ converging to $+\infty$ such that $\pi \circ \phi_{t}(v)$ belongs to $\mathscr{N}_{\epsilon} C$ for every $t$ in $\left[t_{n}, t_{n}+g\left(t_{n}\right)\right]$.

Define the penetration map $\mathfrak{p}: T^{1} M \times \mathbb{R} \rightarrow[0,+\infty]$ into $\mathscr{N}_{\epsilon} C$ by $\mathfrak{p}(v, t)=0$ if $\pi \circ \phi_{t}(v) \notin \mathscr{N}_{\epsilon} C$, and otherwise $\mathfrak{p}(v, t)$ is the maximal length of an interval $I$ in $\mathbb{R}$ containing $t$ such that $\pi \circ \phi_{s}(v) \in \mathscr{N}_{\epsilon} C$ for every $s$ in $I$. We refer to $[\mathbf{P P 1}]$ for (many) other ways to measure the penetration of a geodesic line in the $\epsilon$-neighborhood of $C$.

Corollary 1.2. For $\mu$-almost every $v \in T^{1} M$,

$$
\limsup _{t \rightarrow+\infty} \frac{\mathfrak{p}(v, t)}{\log t}=\frac{1}{h} .
$$

When $M$ has constant curvature, and after a geometric translation, Theorem 1.1 and Corollary 1.2 follow from known results (see for instance $[\mathbf{D M P V}]$, as well as the recent $[\mathbf{B V}]$, where the methods are very different).

We also prove a Khintchine-type theorem for geodesic lines spiraling around totally geodesic submanifolds. For the sake of simplicity in this introduction, we only formulate it for real hyperbolic manifolds, see Theorem 5.3 for a more general statement.

Theorem 1.3. Assume furthermore that $M$ is a real hyperbolic $n$ manifold, and let $C$ be a closed embedded totally geodesic submanifold of dimension $k \geq 1$. Let $\epsilon>0$ be small enough.

If $\int_{1}^{+\infty} e^{-(n-k) g(t)} d t$ converges (resp. diverges), then for $\mu$-almost no (resp. every) $v \in T^{1} M$, there exist positive times $\left(t_{n}\right)_{n \in \mathbb{N}}$ converging to $+\infty$ such that $\pi \circ \phi_{t}(v)$ belongs to $\mathscr{N}_{\epsilon} C$ for every $t$ in $\left[t_{n}, t_{n}+g\left(t_{n}\right)\right]$.

Besides totally geodesic submanifolds, one could also measure the asymptotic spiraling of geodesic lines around other convex subsets, in particular in hyperbolic 3-manifolds.

Theorem 1.4. Assume furthermore that $M=\Gamma \backslash \mathbb{H}_{\mathbb{R}}^{3}$ is a hyperbolic 3-manifold, and let $\Gamma_{0}$ be a quasi-fuschian subgroup of $\Gamma$. Let $\delta_{0}$ be the Hausdorff dimension of the limit set $\Lambda \Gamma_{0}$ of $\Gamma_{0}$, and let $C$ be the image in $M$ of the convex hull $\mathscr{C} \Lambda \Gamma_{0}$ of $\Lambda \Gamma_{0}$ in $\mathbb{H}_{\mathbb{R}}^{3}$. Assume that $\gamma \mathscr{C} \Lambda \Gamma_{0} \cap \mathscr{C} \Lambda \Gamma_{0}$ is empty for every $\gamma \in \Gamma-\Gamma_{0}$. Let $\epsilon>0$ be small enough.

If $\int_{1}^{+\infty} e^{-\left(2-\delta_{0}\right) g(t)} d t$ converges (resp. diverges), then for $\mu$-almost no (resp. every) $v \in T^{1} M$, there exist positive times $\left(t_{n}\right)_{n \in \mathbb{N}}$ converging to $+\infty$ such that $\pi \circ \phi_{t}(v)$ belongs to $\mathscr{N}_{\epsilon} C$ for every $t$ in $\left[t_{n}, t_{n}+g\left(t_{n}\right)\right]$.

All these results follow from our main result, Theorem 5.3, which is much stronger than the above ones. We do not require $M$ to be compact. The first two statements above remain valid when $M$ is complete, non elementary, with a negative upper bound on its sectional curvature, up 
to replacing $h$ by the critical exponent $\delta$ of the fundamental group $\Gamma$ of $M$ acting on a universal Riemannian covering $\widetilde{M}$ of $M$, and under some assumptions on $\Gamma$. Under these hypotheses on $M$, Theorem 1.4 is still valid, up to replacing $\mathbb{H}_{\mathbb{R}}^{3}$ by $\widetilde{M}, \Gamma_{0}$ by a malnormal infinite index convex-cocompact subgroup of $\Gamma$ with critical exponent $\delta_{0}$, and $2-\delta_{0}$ by $\delta-\delta_{0}$. We may also replace the Bowen-Margulis measure on $T^{1} M$ by some other measures, see the comment after Lemma 2.4.

Furthermore, we do not need $\widetilde{M}$ to be a manifold, Theorem 5.3 is valid in general CAT $(-1)$ spaces, for instance in hyperbolic buildings (i.e. Tits buildings modeled on a hyperbolic reflection group), see [Bou2, GP , HaP] for examples. Corollary 5.5 is an example of an application. In this introduction, we quote two results in the setting of trees. The first one will be proved in Section 5 . Let $E[\cdot]$ be the integer part map.

Proposition 1.5. Let $T$ be a locally finite tree, and $\operatorname{Aut}(T)$ be its locally compact group of automorphisms, such that Aut $(T) \backslash T$ is finite. Let $\Gamma$ be a lattice in $\operatorname{Aut}(T)$ acting without inversion on $T$. Let $\Gamma \backslash \mathscr{G T}$ be the quotient by $\Gamma$ of the space $\mathscr{G} T$ of isometric maps $\ell: \mathbb{R} \rightarrow T$, endowed with its geodesic flow $\left(\phi_{t}\right)_{t \in \mathbb{R}}$ (the action of $\mathbb{R}$ by translations at the source). Let $\mu$ be the Bowen-Margulis measure for the action of $\left(\phi_{t}\right)_{t \in \mathbb{R}}$ on $\Gamma \backslash \mathscr{G} T$, and $h$ be the Hausdorff dimension of the space of ends of $T$. Let $C$ be a cycle in the graph $\Gamma \backslash T$ with $L$ edges.

If $\int_{1}^{+\infty} e^{-\frac{h}{L} g(t)} d t$ converges (resp. diverges), then for $\mu$-almost no (resp. every) $\ell \in \Gamma \backslash \mathscr{G} T$, there exist positive times $\left(t_{n}\right)_{n \in \mathbb{N}}$ converging to $+\infty$ such that the path $t \mapsto \ell(t)$, starting at time $t_{n}$, turns around $C$ at least $E\left[g\left(t_{n}\right)\right]$ times.

The next result (see Section 6) uses the Bruhat-Tits tree of the algebraic group $\mathrm{SL}_{2}$ over the local field $\widehat{K}=\mathbb{F}_{q}\left(\left(X^{-1}\right)\right)$ of formal Laurent series in the variable $X^{-1}$ over the finite field $\mathbb{F}_{q}$. Let $\mu$ be a Haar measure of $\widehat{K}$. Let $|\cdot|_{\infty}$ be the absolute value of $\widehat{K}$. Recall that an element of $\widehat{K}$ is irrational if it does not belong to the subfield $\mathbb{F}_{q}(X)$ of rational fractions over $\mathbb{F}_{q}$, and is quadratic if it is a solution of a quadratic equation with coefficients in $\mathbb{F}_{q}(X)$. The group $\mathrm{SL}_{2}\left(\mathbb{F}_{q}[X]\right)$ acts by homographies on the set of quadratic irrational elements of $\widehat{K}$, and two of these are congruent if they are in the same orbit. For every irrational quadratic element $\alpha$ in $\widehat{K}$, define $h(\alpha)=\left|\alpha-\alpha^{*}\right|_{\infty}^{-1}$, where $\alpha^{*}$ is the Galois conjugate of $\alpha$, which measures the complexity of $\alpha$ (see Section 6). In [HP5], we proved a 0-1 measure result for the Diophantine approximation of elements of $\widehat{K}$ by rational ones. The following result (see Section 6) is an analogous one for the Diophantine approximation of elements of $\widehat{K}$ by quadratic irrational ones.

Theorem 1.6. Let $\varphi: \mathbb{R}_{+} \rightarrow \mathbb{R}_{+}^{*}$ be a map with $u \mapsto \log \varphi\left(e^{u}\right)$ Lipschitz. If the integral $\int_{1}^{+\infty} \varphi(t) / t d t$ diverges (resp. converges), then 
for $\mu$-almost every $x \in \widehat{K}$,

$$
\liminf \frac{h(\beta)}{\varphi(h(\beta))}|x-\beta|_{\infty}=0(\text { resp. }=+\infty),
$$

where the lower limit is taken over the quadratic irrational elements $\beta$ in $\widehat{K}$, in any congruence class, with $h(\beta) \rightarrow+\infty$.

For other number theoretic applications of the results of this paper and of $[\mathbf{P P 1}]$, we refer to $[\mathbf{P P 2}]$.

We first start in Section 2 by recalling properties of the CAT $(-1)$ spaces $X$ and their spaces at infinity $\partial_{\infty} X$. We introduce, for every non empty closed convex subset $C$ of $X$, a nice new distance-like map $d_{C}$ on $\partial_{\infty} X-\partial_{\infty} C$. It generalizes Gromov's distance when $C$ is reduced to a point (see for instance [Bou1]), or Hamenstädt's distance when $C$ is a horoball (see [Ham] [HP1, Appendix]).

In Section 3, we present the main technical tool of this paper, a geometric avatar of the Borel-Cantelli lemma.

This tool will also be used in Appendix A, which is joint work with C. S. Aravinda, to prove other approximation results, both of Khintchine-type and logarithm law-type, expressing how exactly close to a given point almost every geodesic line passes. In particular, we give in the appendix a new proof of the following result by F. Maucourant [Mau]

Theorem 1.7 (Maucourant). Assume furthermore that $M$ has constant curvature -1 and dimension $n$, and let $x_{0} \in M$. Then for Liouvillealmost every $v$ in $T^{1} M$,

$$
\limsup _{t \rightarrow+\infty} \frac{-\log d\left(\pi \circ \phi_{t}(v), x_{0}\right)}{\log t}=\frac{1}{n-1} .
$$

See Theorem A.3 in the appendix for a more general statement (valid in variable negative curvature) about the approximation of given points by almost every geodesic lines. The appendix can be read independently of the sections $4,5,6$.

In Section 4, we start by explaining the general situation covering all results 1.1-1.6. We prove some new estimates on the relative geometry of the convex hulls of subgroups of a discrete group of isometries of a $\operatorname{CAT}(-1)$ metric space $X$. Among the new geometric information (possibly useful for later applications), we give in Theorem 4.4 a fluctuating density result. It explains the variation in $\epsilon$ of the mass for a conformal density of the $\epsilon$-neighborhood of the limit set of a convexcocompact subgroup. In Section 5, we then prove our main result, Theorem 5.3, as a geometric consequence of Theorem 4.6. This last result describes approximation properties of limit points in the sphere at infinity of $X$ by various invariant subsets, and could have other applications. 
Khintchine-type theorems and their logarithm law-type corollaries for the spiraling of geodesic lines around cusps were obtained by D. Sullivan [Sul] when $M$ has finite volume and constant curvature (see the nice complements by A. Haas [Haa1, Haa2] for surfaces), by D. Kleinbock and G. Margulis $[\mathbf{K M}]$ if $M$ is a finite volume locally symmetric space, by B. Stratmann and S. L. Velani $[\mathbf{S V}]$ (see also for instance [DMPV, $\mathbf{B V}]$ ) if $M$ is geometrically finite with constant curvature, and by the authors [HP4] if $M$ is geometrically finite with variable curvature. In this paper, which requires many new geometric inputs, our intellectual debt to D. Sullivan's work is still important.

Acknowledgements. The second author acknowledges the support of the University of Georgia at Athens for fruitful visits. We also thank V. Kleptsyn and M. Pollicott for their comments, F. Haglund for Remark 5.4, and the referee for many improvements in the writing of this paper.

\section{On convexity properties of CAT(-1)-spaces and their discrete subgroups}

We refer for instance to $[\mathbf{B o u} \mathbf{1}, \mathbf{B H}]$ for the definitions and basic properties of $\mathrm{CAT}(-1)$ metric spaces, their horospheres and their discrete groups of isometries. The new result in this section is the contruction of the distance-like map $d_{C}$ for a convex subset $C$ in subsection 2.2 .

2.1. Generalities. Let $X$ be a proper CAT $(-1)$ geodesic metric space. Its boundary at infinity is denoted by $\partial_{\infty} X$. The space of geodesic lines $\ell: \mathbb{R} \rightarrow X$ in $X$, with the compact-open topology, is denoted by $\mathscr{G} X$. The geodesic flow on $\mathscr{G} X$ is the action of $\mathbb{R}$ by translation at the source. For every base point $x_{0}$ in $X$, the space $\mathscr{G} X$ identifies with $\left(\left(\partial_{\infty} X \times \partial_{\infty} X\right)-\Delta\right) \times \mathbb{R}$, where $\Delta$ is the diagonal in $\partial_{\infty} X \times \partial_{\infty} X$, by the map which associates to a geodesic line $\ell$ the triple $(\ell(-\infty), \ell(+\infty), t)$ of the points at infinity of $\ell$ and the algebraic distance $t$ on $\ell$ (oriented from $\ell(-\infty)$ to $\ell(+\infty)$ ) between $\ell(0)$ and the closest point of $\ell$ to $x_{0}$. This parametrization (called Hopf's) differs from the one defined by another base point $x_{0}^{\prime}$ only by an additive term on the third factor (invariant under the geodesic flow). When $X$ is a Riemannian manifold, the map $T^{1} X \rightarrow \mathscr{G} X$ which associates to $v \in T^{1} X$ the geodesic line $t \mapsto \pi \circ \phi_{t}(v)$, with $\pi: T^{1} X \rightarrow X$ the canonical projection, is an homeomorphism equivariant with respect to the actions of the isometry group of $X$ and of the geodesic flows on $T^{1} X$ and $\mathscr{G} X$.

For every $\xi$ in $\partial_{\infty} X$, the Buseman function at $\xi$ is the map $\beta_{\xi}$ from $X \times X$ to $\mathbb{R}$ defined by

$$
\beta_{\xi}(x, y)=\lim _{t \rightarrow+\infty} d\left(x, \xi_{t}\right)-d\left(y, \xi_{t}\right)
$$


for any geodesic ray $t \mapsto \xi_{t}$ ending at $\xi$. For every subset $A$ of $X$ and every point $x$ in $X \cup \partial_{\infty} X$, we denote by $\mathscr{O}_{x} A$ the shadow of $A$ seen from $x$, i.e. the set of points at infinity of the geodesic rays or lines starting from $x$ and meeting $A$.

The following result, needed only in Appendix A, is Proposition 3.1 of [HP2] (whose proof of the left inclusion is valid under the only assumptions below).

Lemma 2.1. Let $\rho$ be a geodesic ray in $X$, with $x=\rho(0)$ and $\xi=$ $\rho(+\infty)$.

(1) For every $c, t>0$, the ball $B_{d_{x}}\left(\xi, c e^{-t}\right)$ for the visual distance (see Example 1 below) $d_{x}$ on $\partial_{\infty} X$ is contained in the shadow $\mathscr{O}_{x}(B(\rho(t), c))$.

(2) If $X$ is a Riemannian manifold with sectional curvature $-a^{2} \leq$ $K \leq-1$, where $a \geq 1$, then there exists $\kappa_{1}, \kappa_{2}, \kappa_{3}>0$ such that for every $\left.c \in] 0, \kappa_{3}\right]$ and every $t \geq \kappa_{2}$, the shadow $\mathscr{O}_{x}(B(\rho(t), c))$ is contained in the ball $B_{d_{x}}\left(\xi, \kappa_{1} c^{\frac{1}{a}} e^{-t}\right)$.

Given a point at infinity $\xi \in \partial_{\infty} X$ and a horosphere $H$ centered at $\xi$, let $d_{\xi, H}, d_{\xi, H}^{\prime}:\left(\partial_{\infty} X-\{\xi\}\right)^{2} \rightarrow \mathbb{R}$ be the following maps. Let $\eta, \eta^{\prime} \in \partial_{\infty} X-\{\xi\}$. Let $t \mapsto \eta_{t}$ and $t \mapsto \eta_{t}^{\prime}$ be the geodesic lines starting from $\xi$, crossing through $H$ at time $t=0$, and converging to $\eta$ and $\eta^{\prime}$, respectively. Define the Hamenstädt distance (see $[\mathbf{H a m}][\mathbf{H P 1}$, Appendix])

$$
d_{\xi, H}\left(\eta, \eta^{\prime}\right)=\lim _{t \rightarrow+\infty} e^{\frac{1}{2} d\left(\eta_{t}, \eta_{t}^{\prime}\right)-t},
$$

which is a distance inducing the original topology on $\partial_{\infty} X-\{\xi\}$. The cuspidal distance (see $[\mathbf{H P 3}]) d_{\xi, H}^{\prime}$ is defined as follows: If $\eta \neq \eta^{\prime}$, then $-\log \left(2 d_{\xi, H}^{\prime}\left(\eta, \eta^{\prime}\right)\right)$ is the signed distance, along the geodesic line ]$\xi, \eta$ [ oriented from $\xi$ to $\eta$, from $H$ to the horosphere centered at $\eta$ and meeting the geodesic line $] \xi, \eta^{\prime}$ [ in exactly one point. Though not necessarily an actual distance, $d_{\xi, H}^{\prime}$ is equivalent to the Hamenstädt distance (see [HP3, Rem. 2.6]).

2.2. A distance-like map at infinity relative to a convex subset. Let $C$ be a non empty closed convex subset of $X$. (Recall that a subset $C$ in a CAT $(-1)$ metric space is convex if $C$ contains the geodesic segment between any two points in $C$.) We denote by $\partial_{\infty} C$ its set of points at infinity (the intersection with $\partial_{\infty} X$ of the closure of $C$ in $X \cup \partial_{\infty} X$ ), and by $\partial C$ its boundary in $X$. For every $\xi$ in $X \cup \partial_{\infty} X$, we define the closest point to $\xi$ on the convex set $C$, denoted by $\pi_{C}(\xi)$, to be the following point $p$ in $C \cup \partial_{\infty} C$ : If $\xi \in X$, then $p$ belongs to $C$ and minimizes the distance between $x$ and a point of $C$; if $\xi \in \partial_{\infty} X-\partial_{\infty} C$, then $p$ belongs to $C$ and the (closed) horoball centered at $\xi$ whose horosphere contains $p$ meets $C$ exactly at $p$; if $\xi \in \partial_{\infty} C$, then we define $p=\xi$. The point $p$ exists, is unique, and $\pi_{C}: X \cup \partial_{\infty} X \rightarrow C \cup \partial_{\infty} C$ is continuous, by 
the properties of CAT $(-1)$-spaces. For every isometry $\gamma$ of $X$, we have $\pi_{\gamma C}=\gamma \circ \pi_{C} \circ \gamma^{-1}$. When $X$ is a Riemannian manifold, $\pi_{C}$ is (outside $\left.\partial_{\infty} C\right)$ the orthogonal projection on $C$.

Let us define a distance-like map $d_{C}$ on $\partial_{\infty} X-\partial_{\infty} C$. For every $\xi, \eta$ in $\partial_{\infty} X-\partial_{\infty} C$, let $t \mapsto \xi_{t}$ and $t \mapsto \eta_{t}$ be geodesic rays, starting at time 0 from the closest points to $\xi$ and $\eta$ on $C$, and converging to $\xi$ and $\eta$ respectively. Define

$$
d_{C}(\xi, \eta)=\lim _{t \rightarrow+\infty} e^{\frac{1}{2} d\left(\xi_{t}, \eta_{t}\right)-t}=\lim _{t \rightarrow+\infty} e^{\frac{1}{2}\left(d\left(\xi_{t}, \eta_{t}\right)-d\left(\xi_{t}, \pi_{C}(\xi)\right)-d\left(\eta_{t}, \pi_{C}(\eta)\right)\right)} .
$$

Note that these limits exist, and the second equality holds for all geodesic rays $t \mapsto \xi_{t}$ and $t \mapsto \eta_{t}$ converging to $\xi$ and $\eta$, respectively. For every isometry $\gamma$ of $X$, we have

$$
d_{\gamma C}(\gamma \xi, \gamma \eta)=d_{C}(\xi, \eta) .
$$

In particular, any isometry of $X$ preserving $C$ preserves $d_{C}$. For every $\epsilon>0$, the (closed) $\epsilon$-neighborhood $\mathscr{N}_{\epsilon} C$ of $C$ in $X$ is still convex with $\partial_{\infty}\left(\mathscr{N}_{\epsilon} C\right)=\partial_{\infty} C$, and

$$
d_{\mathscr{N}_{\epsilon} C}(\xi, \eta)=e^{\epsilon} d_{C}(\xi, \eta) .
$$

Examples. (1) If $C$ is reduced to a point $x$ in $X$, then $d_{C}=d_{x}$ is the usual visual distance seen from $x$ on $\partial_{\infty} X$ (see for instance [Bou1]).

(2) If $C$ is a (closed) ball of center $x$ and radius $r$, then $d_{C}=e^{r} d_{x}$, as $C=\mathscr{N}_{r}\{x\}$.

(3) If $C$ is a (closed) horoball with point at infinity $\xi$ and boundary horosphere $H$, then $d_{C}=d_{\xi, H}$ is the Hamenstädt distance on $\partial_{\infty} X-\{\xi\}$ as recalled above. If $\left(C_{n}\right)_{n \in \mathbb{N}}$ is a sequence of balls converging uniformly on compact subsets of $X$ to an horoball $C$, then the maps $d_{C_{n}}$ converge uniformly on compact subsets of $\partial X-\{\xi\}$ to $d_{C}$.

(4) If $X$ is a metric tree, then it is easy to prove that, for every $\xi, \eta$ in $\partial_{\infty} X-\partial_{\infty} C$,

$$
d_{C}(\xi, \eta)= \begin{cases}e^{\frac{1}{2} d\left(\pi_{C}(\xi), \pi_{C}(\eta)\right)} & \text { if } \pi_{C}(\xi) \neq \pi_{C}(\eta) \\ e^{-d\left(\pi_{C}(\xi), q\right)} & \text { if } \pi_{C}(\xi)=\pi_{C}(\eta) \text { and } \\ & {\left[\pi_{C}(\xi), \xi\left[\cap \left[\pi_{C}(\eta), \eta\left[=\left[\pi_{C}(\xi), q\right] .\right.\right.\right.\right.}\end{cases}
$$

In particular, in a sufficiently small neighborhood of any point $\xi_{0}$ in $\partial_{\infty} X-\partial_{\infty} C$, the map $d_{C}$ then coincides with the visual distance $d_{\pi_{C}\left(\xi_{0}\right)}$.

(5) Let $X$ be the real hyperbolic $n$-space $\mathbb{H}_{\mathbb{R}}^{n}$, and let $C$ be a complete totally geodesic submanifold of dimension $k$ with $0<k<n$. Let $x_{0}$ be a point in $C$, and $S_{x_{0}}\left(C^{\perp}\right)$ be the sphere of unit tangent vectors at $x_{0}$ that are perpendicular to $C$, endowed with the angular distance $\left(v, v^{\prime}\right) \mapsto$ $\angle_{x_{0}}\left(v, v^{\prime}\right)$. Note that the standard Euclidean distance on $S_{x_{0}}\left(C^{\perp}\right)$ is given by $\left(v, v^{\prime}\right) \mapsto 2 \sin \frac{\iota x_{0}\left(v, v^{\prime}\right)}{2}$. For every $\xi \in \partial_{\infty} X-\partial_{\infty} C$, let $\pi_{C}^{\prime}(\xi)$ be the parallel transport to $x_{0}$, along a geodesic line through $x_{0}$ and $\pi_{C}(\xi)$, of the unit tangent vector at $\pi_{C}(\xi)$ of the geodesic ray $\left[\pi_{C}(\xi), \xi[\right.$. 
We thus get a map $\pi_{C}^{\prime}: \partial_{\infty} X-\partial_{\infty} C \rightarrow S_{x_{0}}\left(C^{\perp}\right)$. In particular, the $\operatorname{map}\left(\pi_{C}, \pi_{C}^{\prime}\right): \partial_{\infty} X-\partial_{\infty} C \rightarrow C \times S_{x_{0}}\left(C^{\perp}\right)$ is a homeomorphism.

Lemma 2.2. For every $\xi, \eta$ in $\partial_{\infty} X-\partial_{\infty} C$,

$$
d_{C}(\xi, \eta)=\sqrt{\sinh ^{2} \frac{d\left(\pi_{C}(\xi), \pi_{C}(\eta)\right)}{2}+\sin ^{2} \frac{\angle_{x_{0}}\left(\pi_{C}^{\prime}(\xi), \pi_{C}^{\prime}(\eta)\right)}{2}} .
$$

Proof. Let $\rho=d\left(\pi_{C}(\xi), \pi_{C}(\eta)\right)$ and let $\theta=\angle_{x_{0}}\left(\pi_{C}^{\prime}(\xi), \pi_{C}^{\prime}(\eta)\right)$. We have to prove that $d_{C}(\xi, \eta)=\frac{1}{2} \sqrt{e^{\rho}+e^{-\rho}-2 \cos \theta}$. This last formula follows from an easy computation using the picture below. Recall that $\sinh b=1 / \tan \alpha$, where $b$ is the hyperbolic length of the arc of any half-circle perpendicular to the horizontal plane between the angles $\alpha$ and $\frac{\pi}{2}$ in the upper halfspace model of $\mathbb{H}_{\mathbb{R}}^{3}$ (see [Bea, page 145]).

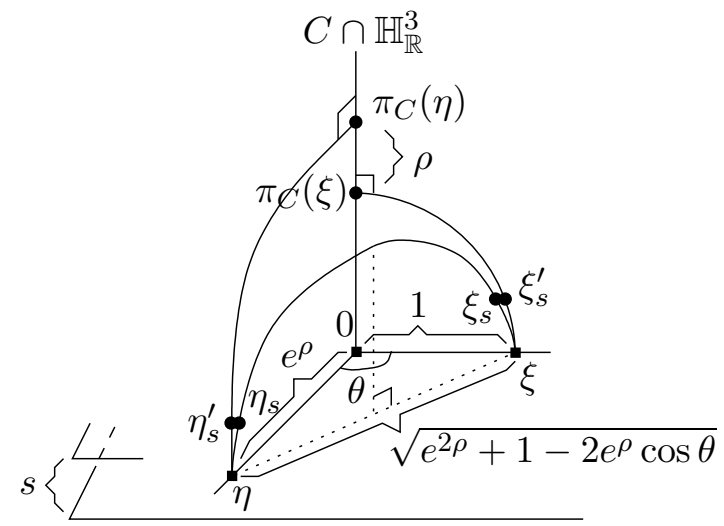

Take a copy of $\mathbb{H}_{\mathbb{R}}^{3}$ containing $\xi, \eta$ and a geodesic line passing through $\pi_{C}(\xi), \pi_{C}(\eta)$. Use the upper halfspace model of $\mathbb{H}_{\mathbb{R}}^{3}$ where this geodesic line is a vertical line between 0 and $\infty$, with $\pi_{C}(\eta)$ above $\pi_{C}(\xi)$. Scale such that the Euclidean distance between 0 and $\xi$ is 1 . Consider the points $\eta_{s}^{\prime}, \eta_{s}, \xi_{s}^{\prime}, \xi_{s}$ at Euclidean height $s$ close to 0 on respectively $\left[\pi_{C}(\eta), \eta[,] \eta, \xi\left[\right.\right.$ close to $\eta,\left[\pi_{C}(\xi), \xi[,] \eta, \xi\left[\right.\right.$ close to $\xi$, so that $d_{C}(\xi, \eta)$ is equal to

$$
\lim _{s \rightarrow 0} e^{\frac{1}{2}\left(d\left(\xi_{s}, \eta_{s}\right)-d\left(\xi_{s}^{\prime}, \pi_{C}(\xi)\right)-d\left(\eta_{s}^{\prime}, \pi_{C}(\eta)\right)\right) .}
$$

Now just use several times the previously mentionned formula $\sinh b=$ $1 / \tan \alpha$.

In particular, if $X=\mathbb{H}_{\mathbb{R}}^{2}$, if $C$ is a geodesic line and if $\xi, \eta$ are in the same component of $\partial_{\infty} X-\partial_{\infty} C$, then

$$
d_{C}(\xi, \eta)=\sinh \frac{d\left(\pi_{C}(\xi), \pi_{C}(\eta)\right)}{2} .
$$

By taking $a, b, c$ in the same component of $\partial_{\infty} X-\partial_{\infty} C$ such that $d\left(\pi_{C}(a), \pi_{C}(b)\right)=d\left(\pi_{C}(b), \pi_{C}(c)\right)=\frac{1}{2} d\left(\pi_{C}(a), \pi_{C}(c)\right)$ are big enough, we see that $d_{C}$ does not satisfy the triangle inequality, hence is not a distance. 
After these examples, let us go back to the general situation on $X, C$, and let us prove some results saying that at least on compact subsets, the map $d_{C}$ behaves quite like a distance.

Lemma 2.3. (1) For every $x_{0}$ in $X$, for every compact subset $K$ of $\partial_{\infty} X-\partial_{\infty} C$, there exists a constant $c_{K}>0$ such that for every $\xi, \eta$ in $K$, we have

$$
\frac{1}{c_{K}} d_{x_{0}}(\xi, \eta) \leq d_{C}(\xi, \eta) \leq c_{K} d_{x_{0}}(\xi, \eta) .
$$

(2) For every $\xi$ in $\partial_{\infty} X-\partial_{\infty} C$, the map $\eta \mapsto d_{C}(\xi, \eta)$ is proper on $\partial_{\infty} X-\partial_{\infty} C$.

(3) For every $\xi, \eta$ in $\partial_{\infty} X-\partial_{\infty} C$,

$$
(3-2 \sqrt{2}) e^{\frac{1}{2} d\left(\pi_{C}(\xi), \pi_{C}(\eta)\right)} e^{-d(C,] \xi, \eta[)} \leq d_{C}(\xi, \eta) \leq e^{\frac{1}{2} d\left(\pi_{C}(\xi), \pi_{C}(\eta)\right)} .
$$

(4) There exist universal constants $c, c^{\prime}>0$ such that for every $\xi, \eta$ in $\partial_{\infty} X-\partial_{\infty} C$, if $d_{C}(\xi, \eta) \leq c$, then $C$ and the geodesic line $] \xi, \eta[$ are disjoint, and

$$
\frac{1}{c^{\prime}} e^{-d(C,] \xi, \eta[)} \leq d_{C}(\xi, \eta) \leq c^{\prime} e^{-d(C,] \xi, \eta[)} .
$$

Note that by hyperbolicity, $\min \left\{d\left(\pi_{C}(\xi), \pi_{C}(\eta)\right), d(C,] \xi, \eta[)\right\}$ is, for every $\xi, \eta$, less than a universal constant.

Proof. For every $x_{0}$ in the convex subset $C$ and $\xi$ in $\partial_{\infty} X-\partial_{\infty} C$, by the triangle inequality and the $\operatorname{CAT}(-1)$ inequality, we have

$$
d\left(\xi_{t}, x_{0}\right) \leq d\left(\xi_{t}, \pi_{C}(\xi)\right)+d\left(x_{0}, \pi_{C}(\xi)\right) \leq d\left(\xi_{t}, x_{0}\right)+2 \log (1+\sqrt{2}),
$$

with $\xi_{t}$ as above. Hence for every $\xi, \eta$ in $\partial_{\infty} X-\partial_{\infty} C$,

$$
(3-2 \sqrt{2}) d_{x_{0}}(\xi, \eta) \leq d_{C}(\xi, \eta) e^{-\frac{1}{2}\left(d\left(x_{0}, \pi_{C}(\xi)\right)+d\left(x_{0}, \pi_{C}(\eta)\right)\right)} \leq d_{x_{0}}(\xi, \eta) .
$$

The first result easily follows. By taking $x_{0}=\pi_{C}(\xi)$ in the lower bound of Equation (2), the second assertion also follows. The upper bound in the third assertion follows by the definition of $d_{C}(\xi, \eta)$ and the triangular inequality.

By the triangle inequality, $d_{x_{0}}(\xi, \eta) \geq e^{-d\left(x_{0},\right] \xi, \eta[)}$. Hence, by taking $x_{0}$ in Equation (2) to be the closest point of $C$ to $] \xi, \eta[$ if $C$ and $] \xi, \eta[$ are disjoint, or any point in $C \cap] \xi, \eta[$ otherwise, and by using again the triangle inequality, the lower bound in the third assertion follows.

The last assertion follows by standard techniques of approximation by trees (see for example [GH, page 33]). $\quad$ q.e.d.

In particular, the non negative symmetric map $d_{C}$ vanishes on and only on the diagonal of $\left(\partial_{\infty} X-\partial_{\infty} C\right)^{2}$. But as mentioned above, $d_{C}$ is not always a distance.

It also follows from Lemma 2.3 (1) that the uniform structure (see for instance $[\mathbf{B o u}]$ ) defined (restricted to compact subsets) by the family 
$\left(\left\{(x, y) \in\left(\partial_{\infty} X-\partial_{\infty} C\right)^{2}: d_{C}(\xi, \eta) \leq \epsilon\right\}\right)_{\epsilon>0}$ is isomorphic (on compacts subsets) to the uniform structure defined by the distance $d_{x_{0}}$.

Remark. Though we will not need it in this paper, we prove a formula expressing the distance-like map $d_{C}$, when $C=L$ is a geodesic line with endpoints $L_{-}, L_{+}$, in terms of the Hamenstädt distance and the cuspidal distance: For every $\xi, \eta$ in $\partial_{\infty} X-\left\{L_{-}, L_{+}\right\}$, for every horosphere $H$ small enough centered at $L_{-}$,

$$
d_{C}(\xi, \eta)=\frac{d_{L_{-}, H}(\xi, \eta)}{2\left(d_{L_{-}, H}^{\prime}\left(\xi, L_{+}\right) d_{L_{-}, H}^{\prime}\left(\eta, L_{+}\right)\right)^{\frac{1}{2}}} .
$$

Proof. Let $H_{\xi}$ (resp. $H_{\eta}$ ) be the horosphere centered at $\xi$ (resp. $\eta$ ) passing through $\pi_{C}(\xi)$ (resp. $\pi_{C}(\eta)$ ). Let $h_{\xi}\left(\right.$ resp. $\left.h_{\eta}\right)$ be the intersection point of $H_{\xi}$ (resp. $H_{\eta}$ ) with the geodesic line $] \xi, L_{-}[$(resp. $] \eta, L_{-}[$). Then

$$
\begin{aligned}
d_{C}(\xi, \eta) & =\lim _{t \rightarrow+\infty} e^{\frac{1}{2}\left(d\left(\xi_{t}, \eta_{t}\right)-d\left(\xi_{t}, h_{\xi}\right)-d\left(\eta_{t}, h_{\eta}\right)\right)} \\
& =d_{L_{-}, H}(\xi, \eta) e^{\frac{1}{2}\left(d\left(h_{\xi}, H\right)+d\left(h_{\eta}, H\right)\right)},
\end{aligned}
$$

which proves the result.

q.e.d.

2.3. Patterson-Sullivan-Bowen-Margulis measures. Let $\Gamma$ be a discrete group of isometries of $X$. Its limit set is denoted by $\Lambda \Gamma$, and if $\Lambda \Gamma$ contains at least two points, then the convex hull of $\Lambda \Gamma$ is denoted by $\mathscr{C} \Gamma$. Recall that $\partial_{\infty} \mathscr{C} \Gamma=\Lambda \Gamma$. The critical exponent of $\Gamma$ is the unique number $\delta_{\Gamma}$ in $[0,+\infty]$ such that the Poincaré series $P_{x_{0}, \Gamma}(s)=\sum_{\gamma \in \Gamma} e^{-s d\left(x_{0}, \gamma x_{0}\right)}$ of $\Gamma$ converges for $s>\delta_{\Gamma}$ and diverges for $s<\delta_{\Gamma}$, where $x_{0}$ is any point in $X$. The group $\Gamma$ is called of $d i$ vergence type if its Poincaré series diverges at $s=\delta_{\Gamma}$. The group $\Gamma$ is non elementary if $\Lambda \Gamma$ contains at least three points, and we have then $\delta_{\Gamma}>0$. Note that when $X$ is a Riemannian manifold and $\Gamma$ is torsion free with compact quotient $\Gamma \backslash X$, then the critical exponent $\delta_{\Gamma}$ of $\Gamma$ is the topological entropy of the geodesic flow of $\Gamma \backslash X$ (see for instance $[\operatorname{Man}])$.

If $\delta \in] 0,+\infty[$, a conformal (or Patterson-Sullivan) density of dimension $\delta$ for $\Gamma$ is a family $\left(\mu_{x}\right)_{x \in X}$ of finite Borel measures on $\partial_{\infty} X$, such that

- $\forall \gamma \in \Gamma, \gamma_{*} \mu_{x}=\mu_{\gamma x}$,

- $\forall x, y \in X, \forall \xi \in \partial_{\infty} X, \frac{d \mu_{x}}{d \mu_{y}}(\xi)=e^{-\delta \beta_{\xi}(x, y)}$.

Using Hopf's parametrization with respect to any base point $x_{0}$ of $X$, the (Patterson-Sullivan-)Bowen-Margulis measure associated to this family is the measure $\widetilde{\mu}_{\mathrm{BM}}$ on $\mathscr{G} X$ given by

$$
d \widetilde{\mu}_{\mathrm{BM}}=\frac{d \mu_{x_{0}}(\xi) d \mu_{x_{0}}(\eta) d t}{d_{x_{0}}(\eta, \xi)^{2 \delta}} .
$$


This measure on $\mathscr{G} X$ is independent of $x_{0}$, invariant by the action of $\Gamma$ and by the geodesic flow (and by the time reversal $\ell \mapsto\{t \mapsto \ell(-t)\}$ ), hence defines a measure $\mu_{\mathrm{BM}}$ on $\Gamma \backslash \mathscr{G} X$ which is invariant by the quotient geodesic flow (see for instance [Bou1, Rob]). Note that if $\mu_{\mathrm{BM}}$ is finite, then $\delta=\delta_{\Gamma}$ and $\Gamma$ is of divergence type (see [Rob, page 18-19]).

If $\Gamma$ is of divergence type with a finite non zero critical exponent $\delta$, then (see for instance [Bou1]) there exists a conformal density of dimension $\delta$ for $\Gamma$, which is unique up to a positive scalar factor, and which is ergodic with respect to the action of $\Gamma$ on $\partial_{\infty} X$. The BowenMargulis measure associated to any such conformal family (both on $\mathscr{G} X$ and on $\Gamma \backslash \mathscr{G} X)$ will be called a Bowen-Margulis measure of $\Gamma$ (it is also uniquely defined up to a positive scalar factor). When $X$ is a manifold and $\Gamma$ acts freely on $X$ with compact quotient, the Bowen-Margulis measure on the unit tangent bundle of the compact negatively curved manifold $M=\Gamma \backslash X$, normalized to be a probability measure, is the maximal entropy probability measure for the geodesic flow of $M$ (via the canonical identification of $\mathscr{G} X$ and $\left.T^{1} X\right)$, see for instance [Kai]. When furthermore $X$ has constant curvature, then the Bowen-Margulis measure and the Liouville measure (when both are normalized) coincide on $T^{1} M$.

The following result, which is obvious by definition of the BowenMargulis measure, will be used in Section 5 and Appendix A.

Lemma 2.4. Let $\pi_{+}: \mathscr{G} X \rightarrow \partial_{\infty} X$ be the continuous map $\ell \mapsto$ $\ell(+\infty)$. Let $\widetilde{\mu}_{B M}$ be the Bowen-Margulis measure on $\mathscr{G} X$ associated to a conformal family $\left(\mu_{x}\right)_{x \in X}$ for $\Gamma$. Then the preimage by $\pi_{+}$of a set of measure 0 (resp. $>0$ ) for $\mu_{x}$ (for some (equivalently for any) $x$ in $X$ ) has measure 0 (resp. $>0$ ) for $\widetilde{\mu}_{B M}$.

Besides its invariance under $\Gamma$ and the geodesic flow, and its ergodicity on $\Gamma \backslash \mathscr{G} X$, this is the only property of the Bowen-Margulis measure $\widetilde{\mu}_{\mathrm{BM}}$ on $\mathscr{G} X$ that will be used in this paper. In particular, we may replace $\widetilde{\mu}_{\mathrm{BM}}$ by any other measure satisfying these invariance properties and this lemma, as for instance the Knieper measure (see [Kni]).

2.4. Convex-cocompact subgroups. The group $\Gamma$ is said to be convex-cocompact if $\Lambda \Gamma$ contains at least two points, and if the action of $\Gamma$ on $\mathscr{C} \Gamma$ has compact quotient. In particular, the group generated by a hyperbolic isometry of $X$ is convex-cocompact, with critical exponent 0 . In fact, if $\Gamma$ is convex-cocompact, then its critical exponent is 0 if and only if $\Gamma$ has an index two subgroup generated by a hyperbolic isometry of $X$. Note that if $\Gamma$ is convex-cocompact then $\Gamma$ is of divergence type (see for instance [Bou1, Rob]). 
For every $f, g: \mathbb{N} \rightarrow[0,+\infty$ [, write $f \asymp g$ if there exists a constant $c \geq 1$ such that $\frac{1}{c} f \leq g \leq c f$. For every $x_{0}$ in $X$, if $\Gamma$ is convexcocompact and non elementary, with critical exponent $\delta_{\Gamma}$, then

$$
\operatorname{Card}\left(B\left(x_{0}, n\right) \cap \Gamma x_{0}\right) \asymp e^{\delta_{\Gamma} n}
$$

(see for instance $[\mathbf{R o b}]$, where others, much more general, assumptions on $\Gamma$ are given for this property to hold. This is the case for example when the Bowen-Margulis measure $\mu_{\mathrm{BM}}$ of $\Gamma$ is finite (and the length spectrum is non arithmetic), see [Rob, page 56]).

Lemma 2.5. Let $\Gamma_{0}$ be a convex-cocompact subgroup with infinite index in a discrete group of isometries $\Gamma$ of $X$. Let $\delta_{0}$ and $\delta$ be the critical exponents of $\Gamma_{0}$ and $\Gamma$ respectively. Then $\delta_{0}<\delta$.

Proof. This is well-known (see for instance [Fur] in a special case).

q.e.d.

Recall that the virtual normalizer $\mathrm{N}_{0}$ of a convex-cocompact subgroup $\Gamma_{0}$ of $\Gamma$ is the stabilizer in $\Gamma$ of the limit set $\Lambda \Gamma_{0}$. It contains the normalizer of $\Gamma_{0}$ in $\Gamma$, and it contains $\Gamma_{0}$ with finite index (see for instance [KS, Arz]).

Recall that a subgroup $H$ of a group $G$ is malnormal if, for every $g$ in $G-H$, we have $g H g^{-1} \cap H=\{1\}$. We will say that a subgroup $H$ of a group $G$ is almost malnormal if, for every $g$ in $G-H$, the subgroup $g \mathrm{Hg}^{-1} \cap \mathrm{H}$ is finite. Note that malnormal implies almost malnormal, and that the converse is true if the ambient group is torsion free.

The following result is folklore, we provide a proof because we couldn't find a precise reference.

Proposition 2.6. Let $\Gamma_{0}$ be a convex-cocompact subgroup of a discrete group $\Gamma$ of isometries of $X$, then the following assertions are equivalent.

(1) $\Gamma_{0}$ is almost malnormal in $\Gamma$;

(2) the limit set of $\Gamma_{0}$ is precisely invariant, i.e. for every $\gamma \in \Gamma-\Gamma_{0}$, the set $\Lambda \Gamma_{0} \cap \gamma \Lambda \Gamma_{0}$ is empty;

(3) $\mathscr{C} \Gamma_{0} \cap \gamma \mathscr{C} \Gamma_{0}$ is compact for every $\gamma \in \Gamma-\Gamma_{0}$;

(4) for every $\epsilon>0$, there exists $\kappa=\kappa(\epsilon)>0$ such that, for every $\gamma \in \Gamma-\Gamma_{0}$, we have $\operatorname{diam}\left(\mathscr{N}_{\epsilon} \mathscr{C} \Gamma_{0} \cap \gamma \mathscr{N}_{\epsilon} \mathscr{C} \Gamma_{0}\right) \leq \kappa$.

The convex hull in $X$ of the limit set of a convex-cocompact subgroup is non compact. Hence an almost malnormal convex-cocompact subgroup of $\Gamma$ is equal to its virtual normalizer, by (2).

Proof. As $\partial_{\infty} \mathscr{C} \Gamma_{0}=\Lambda \Gamma_{0}$ and $\gamma \Lambda \Gamma_{0}=\Lambda\left(\gamma \Gamma_{0} \gamma^{-1}\right)$, it is clear that (4) implies (3), which implies (2), which implies (1).

Let us prove that (1) implies (4). Let $C_{0}=\mathscr{C} \Gamma_{0}$ and $\epsilon>0$. Assume by absurd that for every $n$ in $\mathbb{N}$, there exists $\gamma_{n}$ in $\Gamma-\Gamma_{0}$ and $x_{n}, y_{n}$ in $\mathscr{N}_{\epsilon} C_{0} \cap \gamma_{n} \mathscr{N}_{\epsilon} C_{0}$ with $d\left(x_{n}, y_{n}\right) \geq n$. As $\Gamma_{0} \backslash \mathscr{N}_{\epsilon} C_{0}$ is compact and 
the action of $\Gamma_{0}$ is isometric, there exists $R>0$ such that $\Gamma_{0} B(x, R)$ contains $\mathscr{N}_{\epsilon} C_{0}$ for every $x$ in $\mathscr{N}_{\epsilon} C_{0}$. As $\gamma_{n}$ is an isometry, we also have that $\gamma_{n} \Gamma_{0} \gamma_{n}^{-1} B(y, R)$ contains $\gamma_{n} \mathscr{N}_{\epsilon} C_{0}$, for every $y$ in $\gamma_{n} \mathscr{N}_{\epsilon} C_{0}$.

Up to conjugating $\gamma_{n}$ by an element of $\Gamma_{0}$, we may assume that $x_{n}$ stays in a compact subset $K$ of $X$, and we define $K^{\prime}=\mathscr{N}_{R} K$, which is compact. As $\Gamma$ is discrete, the number $N$ of elements $\gamma$ in $\Gamma$, such that $\gamma K^{\prime} \cap K^{\prime}$ is non empty, is finite.

As $\Gamma_{0}$ is convex-cocompact (and discrete), the upper bound of the cardinals of the finite subgroups of $\Gamma_{0}$ is finite. Hence, as $\Gamma_{0}$ is almost malnormal, there exists $N^{\prime} \in \mathbb{N}$ such that for every $\gamma$ in $\Gamma-\Gamma_{0}$, the cardinal of $\gamma \Gamma_{0} \gamma^{-1} \cap \Gamma_{0}$ is at most $N^{\prime}-2$.

Take $n$ in $\mathbb{N}$ with $n>N N^{\prime}$ diam $K^{\prime}$. Subdivide the segment between $x_{n}$ and $y_{n}$ in points $u_{0}=x_{n}, u_{1}, \ldots, u_{N N^{\prime}}=y_{n}$, such that $d\left(u_{k}, u_{k+1}\right)>$ $\operatorname{diam} K^{\prime}$ for $0 \leq k \leq N N^{\prime}-1$. As $K^{\prime}$ contains $B\left(x_{n}, R\right)$ and $x_{n}, y_{n}$ belong to the convex subset $\mathscr{N}_{\epsilon} C_{0} \cap \gamma_{n} \mathscr{N}_{\epsilon} C_{0}$, for $0 \leq k \leq N N^{\prime}$, there exist $\alpha_{k}, \beta_{k}$ in $\Gamma_{0}$ such that $u_{k} \in \alpha_{k} K^{\prime}$ and $u_{k} \in \gamma_{n} \beta_{k} \gamma_{n}^{-1} K^{\prime}$. Note that $\alpha_{k} \neq \alpha_{j}$ if $k \neq j$, as $d\left(u_{k}, u_{j}\right)>\operatorname{diam} K^{\prime}$. By the definition of $N$, there exists $\left(k_{j}\right)_{1 \leq j \leq N^{\prime}}$ with $\alpha_{k_{j}}^{-1} \gamma_{n} \beta_{k_{j}} \gamma_{n}^{-1}=\alpha_{k_{1}}^{-1} \gamma_{n} \beta_{k_{1}} \gamma_{n}^{-1}$ for $1 \leq j \leq N^{\prime}$. Hence $\gamma_{n} \beta_{k_{j}} \beta_{k_{1}}^{-1} \gamma_{n}^{-1}=\alpha_{k_{j}} \alpha_{k_{1}}^{-1}$, for $2 \leq j \leq N^{\prime}$. This contradicts the fact that the cardinal of $\gamma_{n} \Gamma_{0} \gamma_{n}^{-1} \cap \Gamma_{0}$ is at most $N^{\prime}-2$, since the $\alpha_{k_{j}} \alpha_{k_{1}}^{-1}$ for $2 \leq j \leq N^{\prime}$ are pairwise distinct.

q.e.d.

Remark. The fact that the first two assertions are equivalent follows also from the well-known equality

$$
\Lambda \Gamma_{0} \cap \gamma \Lambda \Gamma_{0}=\Lambda\left(\Gamma_{0} \cap \gamma \Gamma_{0} \gamma^{-1}\right),
$$

see for instance [SS, Coro. 3] for a proof in a special case.

\section{A geometric avatar of the Borel-Cantelli lemma}

The main technical tool of this paper is the following result, which is a suitable enhancement of the Borel-Cantelli Lemma.

Theorem 3.1. Let $(Z, \mu)$ be a measured space with $\mu(Z)$ finite, and $\left(B_{i}(\epsilon)\right)_{i \in I, \epsilon \in] 0,+\infty[}$ a family of measurable subsets in $Z$, non-decreasing in $\epsilon$ (for the inclusion), endowed with a map $i \mapsto n_{i}$ from $I$ to $\mathbb{N}$ such that $I_{n}=\left\{i \in I: n_{i}=n\right\}$ is finite for every $n$. Let $f_{1}, f_{2}, f_{3}, f_{4}$ be maps from $\mathbb{N}$ to $] 0,+\infty\left[\right.$ and let $f_{5}$ be a map from $] 0,+\infty[$ to itself. Let $E$ be the (measurable) set of points in $Z$ belonging to infinitely many subsets $B_{i}\left(f_{3}\left(n_{i}\right)\right)$ for $i$ in $I$.

$[A]$ Assume that $f_{3} \leq f_{2}$ and that there exists $c \geq 1$ such that, for every $n$ in $\mathbb{N}, i$ in $I$ and $\left.\epsilon \in] 0, f_{2}\left(n_{i}\right)\right]$, one has Card $I_{n} \leq c f_{1}(n)$ and $\mu\left(B_{i}(\epsilon)\right) \leq c f_{4}\left(n_{i}\right) f_{5}(\epsilon)$. If the series $\sum_{n=0}^{\infty} f_{1}(n) f_{4}(n) f_{5}\left(f_{3}(n)\right)$ converges, then $\mu(E)=0$.

$[B]$ Assume that there exists $c \geq 1$ such that 
(1) $f_{3} \leq f_{2}$,

(2) $\frac{1}{f_{5} \circ f_{2}} \leq f_{4} f_{1}$,

(3) there exist $c^{\prime}, c^{\prime \prime}>1$ such that for every $\epsilon, \epsilon^{\prime}>0$, if $\epsilon^{\prime} \leq c^{\prime} \epsilon$, then $f_{5}\left(\epsilon^{\prime}\right) \leq c^{\prime \prime} f_{5}(\epsilon)$

(4) for every $n$ in $\mathbb{N}$, one has $\frac{1}{c} f_{1}(n) \leq$ Card $I_{n} \leq c f_{1}(n)$,

(5) for every $i$ in $I$ and $\left.\epsilon \in] 0, f_{2}\left(n_{i}\right)\right]$, we have

$$
\frac{1}{c} f_{4}\left(n_{i}\right) f_{5}(\epsilon) \leq \mu\left(B_{i}(\epsilon)\right) \leq c f_{4}\left(n_{i}\right) f_{5}(\epsilon),
$$

(6) for every $n$ in $\mathbb{N}$, the subsets $B_{i}\left(f_{2}(n)\right)$ for $i$ in $I_{n}$ are pairwise disjoint,

(7) for every $i$ and $j$ in $I$ such that $n_{i}<n_{j}$, if the intersection of $B_{j}\left(f_{3}\left(n_{j}\right)\right)$ and $B_{i}\left(f_{3}\left(n_{i}\right)\right)$ is non empty, then $B_{j}\left(f_{2}\left(n_{j}\right)\right)$ is contained in $B_{i}\left(c f_{3}\left(n_{i}\right)\right)$.

If the series $\sum_{n=0}^{\infty} f_{1}(n) f_{4}(n) f_{5}\left(f_{3}(n)\right)$ diverges, then $\mu(E)>0$.

Note that (except for the convergence of the series) every hypothesis of Case [A] is part of a hypothesis (1)-(5) of Case [B]. Hence when checking the hypotheses when we want to apply both cases of this theorem, we will only check the ones of Case $[\mathrm{B}]$.

Proof. For $i$ in $I$ and $n$ in $\mathbb{N}$, let $B_{i}=B_{i}\left(f_{3}\left(n_{i}\right)\right)$ and $A_{n}=\bigcup_{i \in I_{n}} B_{i}$, so that $E=\bigcap_{n \in \mathbb{N}} \bigcup_{k \geq n} A_{k}$.

Under the assumptions of $[\mathrm{A}]$, by the subadditivity of $\mu$, we have the inequality $\mu\left(A_{n}\right) \leq c^{2} f_{1}(n) f_{4}(n) f_{5}\left(f_{3}(n)\right)$. Therefore the end of the proof is standard: If the series $\sum_{n=0}^{\infty} f_{1}(n) f_{4}(n) f_{5}\left(f_{3}(n)\right)$ converges, then the sequence $u_{k}=\sum_{n=k}^{\infty} f_{1}(n) f_{4}(n) f_{5}\left(f_{3}(n)\right)$ tends to 0 , therefore

$$
\mu(E)=\lim _{n \rightarrow \infty} \mu\left(\bigcup_{k=n}^{\infty} A_{k}\right) \leq \lim _{n \rightarrow \infty} c^{2} u_{n}=0 .
$$

Assume now that the assumptions of $[\mathrm{B}]$ hold. We first claim that

$$
f_{1}(n) f_{4}(n) f_{5}\left(f_{3}(n)\right) \leq c^{2} \mu\left(A_{n}\right) .
$$

Indeed, the balls $B_{i}$ for $i$ in $I_{n}$ are pairwise disjoint by (1) and (6), since the subsets $B_{i}(r)$ are non-decreasing in $r$. By the additivity of $\mu$, by the lower bounds in (4) and (5), the inequality $(*)$ hence follows.

In particular, $\sum \mu\left(A_{n}\right)$ diverges if $\sum f_{1}(n) f_{4}(n) f_{5}\left(f_{3}(n)\right)$ diverges.

Now, let $n, m$ be in $\mathbb{N}$ with $n<m$. By the properties (6) and (7), for every $i$ in $I_{n}$, we have

$$
\mu\left(B_{i}\left(c f_{3}\left(n_{i}\right)\right)\right) \geq \operatorname{Card}\left\{j \in I_{m}: B_{j} \cap B_{i} \neq \emptyset\right\} \min _{j \in I_{m}} \mu\left(B_{j}\left(f_{2}(m)\right)\right) .
$$

Hence by (5)

$$
\operatorname{Card}\left\{j \in I_{m}: B_{j} \cap B_{i} \neq \emptyset\right\} \leq \frac{c f_{4}(n) f_{5}\left(c f_{3}(n)\right)}{\frac{1}{c} f_{4}(m) f_{5}\left(f_{2}(m)\right)} \cdot(* *)
$$


Therefore

$$
\begin{aligned}
\mu\left(A_{n} \cap A_{m}\right) & \leq \sum_{i \in I_{n}} \sum_{j \in I_{m}, B_{j} \cap B_{i} \neq \emptyset} \mu\left(B_{j}\right) \\
& \leq c f_{1}(n) \times \frac{c f_{4}(n) f_{5}\left(c f_{3}(n)\right)}{\frac{1}{c} f_{4}(m) f_{5}\left(f_{2}(m)\right)} \times c f_{4}(m) f_{5}\left(f_{3}(m)\right) \\
& \leq c^{4}\left(c^{\prime \prime}\right)^{\frac{\log c}{\log c^{\prime}}+1} f_{1}(n) f_{4}(n) f_{5}\left(f_{3}(n)\right) f_{1}(m) f_{4}(m) f_{5}\left(f_{3}(m)\right) \\
& \leq c^{8}\left(c^{\prime \prime}\right)^{\frac{\log c}{\log c^{\prime}}+1} \mu\left(A_{n}\right) \mu\left(A_{m}\right) .
\end{aligned}
$$

The second inequality follows from $(4),(* *)$ and (5), the third inequality follows from (2) and an iterated application of (3), and the last one from $(*)$.

The following Borel-Cantelli Lemma is well-known (see for instance $[\mathbf{S p r}])$.

Theorem 3.2. Let $(Z, \nu)$ be a probability space. Let $\left(A_{n}\right)_{n \in \mathbb{N}}$ be a sequence of measurable subsets of $Z$ such that there exists a constant $c>0$ with $\nu\left(A_{n} \cap A_{m}\right) \leq c \nu\left(A_{n}\right) \nu\left(A_{m}\right)$ for every distinct integers $n, m$. Let $A_{\infty}=\bigcap_{n \in \mathbb{N}} \bigcup_{k \geq n} A_{k}$. Then $\nu\left(A_{\infty}\right)>0$ if and only if $\sum_{n=0}^{\infty} \nu\left(A_{n}\right)$ diverges.

The assertion [B] of Theorem 3.1 then follows.

q.e.d.

\section{Approximation of limit points}

In this section, we start by describing our general framework: a nice subgroup $\Gamma_{0}$ of a discrete group of isometries $\Gamma$ of a $\mathrm{CAT}(-1)$ space. The main result of this section, Theorem 4.6, explains in a quantitative way the approximation of the limit points of $\Gamma$ by the orbits under $\Gamma$ of the limit points of $\Gamma_{0}$. To prove it, we will check, in a series of results of independent interests, the hypotheses (1)-(7) of Theorem 3.1, our geometric avatar of the Borel-Cantelli Lemma.

Let $X$ be a proper $\mathrm{CAT}(-1)$ geodesic metric space. Let $\Gamma$ be a non elementary discrete group of isometries of $X$, with finite critical exponent $\delta$. Let $\Gamma_{0}$ be an almost malnormal convex-cocompact subgroup of infinite index in $\Gamma$ with critical exponent $\delta_{0}$, and let $C_{0}=\mathscr{C} \Gamma_{0}$. It is likely that the hypothesis "convex-cocompact" could be replaced by "geometrically finite" up to some adaptations, but this would surely make the statements and proofs much more technical, hence we prefer to work under our hypotheses. Let $\pi_{C_{0}}: X \cup \partial_{\infty} X \rightarrow C_{0} \cup \partial_{\infty} C_{0}$ be the closest point map. By Lemma 2.5, the number $\delta_{0}$ belongs to $[0, \delta[$. Moreover, it follows from Section 2 that $C_{0}$ is non compact and that $\Gamma_{0}$ is the stabilizer in $\Gamma$ of $C_{0}$. 
Examples. (1) Let $\gamma_{0}$ be a hyperbolic element of $\Gamma$, let $C_{0}$ be its translation axis and let $\Gamma_{0}$ be the stabilizer of $C_{0}$ (which is virtually infinite cyclic, and infinite cyclic when $\Gamma$ is torsion free). Since $\Gamma$ is non elementary, the subgroup $\Gamma_{0}$ has infinite index. Furthermore, if $\gamma \in \Gamma$ and $\gamma \Gamma_{0} \gamma^{-1} \cap \Gamma_{0}$ is infinite, then $\gamma$ conjugates some hyperbolic element of $\Gamma_{0}$ to another one. The image by an element $\gamma$ in $\Gamma$ of the translation axis of a hyperbolic element $\alpha$ of $\Gamma$ is the translation axis of $\gamma \alpha \gamma^{-1}$. Hence $\gamma$ preserves $C_{0}$, therefore belongs to $\Gamma_{0}$. Therefore $\Gamma_{0}$ is an almost malnormal convex-cocompact subgroup of infinite index in $\Gamma$ with critical exponent $\delta_{0}=0$.

(2) Let $M$ be a complete Riemannian manifold with dimension $n \geq 2$ and sectional curvature at most -1 , and $\pi: X \rightarrow M$ be a universal Riemannian covering, with covering group $\Gamma$. Let $M_{0}$ be a compact connected embedded totally geodesic submanifold in $M$ of dimension $k$ with $1 \leq k \leq n-1$, let $C_{0}$ be a connected component of the preimage of $M_{0}$ in $X$, and let $\Gamma_{0}$ be the stabilizer of $C_{0}$ in $\Gamma$ (with good choices of base points, $\Gamma$ can be identified with the fundamental group of $M$, and $\Gamma_{0}$ with the image in the fundamental group of $M$ of the fundamental group of $M_{0}$ ). Then $\Gamma_{0}$ is an almost malnormal (for instance by Proposition 2.6 (3)) convex-cocompact subgroup of $\Gamma$. If for instance $M$ has finite volume, then $\Gamma$ is non elementary and $\Gamma_{0}$ has infinite index in $\Gamma$. If $M$ has constant sectional curvature -1 , then $\delta=n-1$ and $\delta_{0}=k-1$.

(3) Let $X=\mathbb{H}_{\mathbb{R}}^{3}$ be the real hyperbolic space of dimension 3 , and $\Gamma$ be a Kleinian group. If $\Gamma_{0}$ is a precisely invariant quasi-fuschian subgroup, without parabolic elements, of infinite index in $\Gamma$, then $\Gamma_{0}$ is an almost malnormal (by Proposition 2.6 (2)) convex-cocompact subgroup of infinite index in $\Gamma$.

After these examples, let us proceed. Denote by $R_{0}$ the set of double cosets

$$
R_{0}=\Gamma_{0} \backslash\left(\Gamma-\Gamma_{0}\right) / \Gamma_{0} .
$$

For every $r=[\gamma]$ in $R_{0}$, define

$$
D(r)=d\left(C_{0}, \gamma C_{0}\right) \in[0,+\infty[,
$$

which does not depend on the representative $\gamma$ of $r$. The next result says that the subset $\left\{D(r): r \in R_{0}\right\}$ of $[0,+\infty[$ is discrete, with finite multiplicities.

Lemma 4.1. For every $c \geq 0$, the set of elements $r$ in $R_{0}$ such that $D(r) \leq c$ is finite.

Proof. For every $c \geq 0$, assume that there exists a sequence of pairwise distinct elements $\left(\left[\gamma_{i}\right]\right)_{i \in \mathbb{N}}$ in $R_{0}$ such that $D\left(\left[\gamma_{i}\right]\right) \leq c$ for every $i$. Fix $x_{*}$ in $C_{0}$, and let $D$ be the diameter of $\Gamma_{0} \backslash C_{0}$. For every $i$ in $\mathbb{N}$, let $x_{i}$ in $C_{0}$ and $y_{i}$ in $\gamma_{i} C_{0}$ be any points such that $d\left(x_{i}, y_{i}\right) \leq c+1$. Up 
to replacing $\gamma_{i}$ by another representative of $\left[\gamma_{i}\right]$, we may assume that $d\left(x_{i}, x_{*}\right) \leq D$ and $d\left(y_{i}, \gamma_{i} x_{*}\right) \leq D$. Hence $d\left(x_{*}, \gamma_{i} x_{*}\right) \leq 2 D+c+1$ for every $i$, which contradicts the discreteness of $\Gamma$.

q.e.d.

Proposition 4.2. Assume that Card $\Gamma x \cap B(x, n) \asymp e^{\delta n}$ for some (hence every) $x$ in $X$. Then there exists $N$ in $\mathbb{N}-\{0\}$ such that

$$
\text { Card }\left\{r \in R_{0}: n \leq D(r)<n+N\right\} \asymp e^{\delta n} .
$$

Proof. Since $\delta_{0}<\delta$, the proof is the same as the proof of $[\mathbf{H P 4}$, Theo. 3.4], up to replacing the horoball $H B_{0}$ by $C_{0}$.

q.e.d.

Define $X_{0}=\Gamma_{0} \backslash X$, and $\partial_{\infty} X_{0}=\Gamma_{0} \backslash\left(\partial_{\infty} X-\Lambda \Gamma_{0}\right)$. Since $\Gamma_{0} \backslash C_{0}$ is compact, and since the closest point map is a proper continuous $\Gamma_{0^{-}}$ equivariant map from $\partial_{\infty} X-\Lambda \Gamma_{0}$ to $C_{0}$, the space $\partial_{\infty} X_{0}$ is compact. The distance-like map $d_{C_{0}}$ on $\partial_{\infty} X-\Lambda \Gamma_{0}$ is invariant under $\Gamma_{0}$, and we denote by $d_{0}$ the quotient distance-like map on $\partial_{\infty} X_{0}$, i.e.

$$
d_{0}(\bar{x}, \bar{y})=\inf _{x \in \bar{x}, y \in \bar{y}} d_{C_{0}}(x, y) .
$$

Let $r=[\gamma]$ be an element in $R_{0}$. Define $\Lambda_{r}$ (which does not depend on the representative $\gamma$ of $r$ ) as the image of $\gamma \Lambda \Gamma_{0}$ by the canonical projection $\partial_{\infty} X-\Lambda \Gamma_{0} \rightarrow \partial_{\infty} X_{0}$. By Proposition 2.6 (2), it follows that $\left(\Lambda_{r}\right)_{r \in R_{0}}$ is a family of pairwise disjoint compact subsets of $\partial_{\infty} X_{0}$. For every $\epsilon>0$, define $\mathscr{N}_{r}(\epsilon)$ as the $\epsilon$-neighborhood of $\Lambda_{r}$ in $\partial_{\infty} X_{0}$ for the distance-like map $d_{0}$. Note that $\mathscr{N}_{r}(\epsilon) \subset \mathscr{N}_{r}\left(\epsilon^{\prime}\right)$ if $\epsilon<\epsilon^{\prime}$.

Let $\left(\mu_{x}\right)_{x \in X}$ be a conformal density of dimension $\delta$ for $\Gamma$. Fix a base point $x_{0}$ in $C_{0}$. Define a map $\widetilde{\mu}_{\Gamma_{0} x_{0}}: \mathscr{B} \rightarrow[0,+\infty]$, where $\mathscr{B}$ is the $\sigma$-algebra of Borel subsets of $\partial_{\infty} X-\Lambda \Gamma_{0}$, by

$$
\widetilde{\mu}_{\Gamma_{0} x_{0}}=\sum_{\alpha \in \Gamma_{0}} \mu_{\alpha x_{0}}
$$

Lemma 4.3. The map $\widetilde{\mu}_{\Gamma_{0} x_{0}}$ is a locally finite positive Borel measure on $\partial_{\infty} X-\Lambda \Gamma_{0}$, which is invariant under $\Gamma_{0}$, and absolutely continuous with respect to the restriction to $\partial_{\infty} X-\Lambda \Gamma_{0}$ of $\mu_{x}$ for every $x$ in $X$.

We denote by $\mu_{\Gamma_{0} x_{0}}$ the finite Borel measure on the compact quotient $\partial_{\infty} X_{0}$ of $\partial_{\infty} X-\Lambda \Gamma_{0}$ defined by $\widetilde{\mu}_{\Gamma_{0} x_{0}}$.

Proof. Denote by $s \mapsto P_{x_{0}, \Gamma_{0}}(s)=\sum_{\alpha \in \Gamma_{0}} e^{-s d\left(\alpha x_{0}, x_{0}\right)}$ the Poincaré series of $\Gamma_{0}$ with base point $x_{0}$.

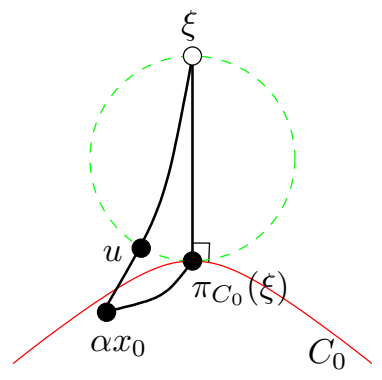


Let $\xi$ be in $\partial_{\infty} X-\Lambda \Gamma_{0}$ and $\alpha$ be in $\Gamma_{0}$. The point $\alpha x_{0}$ belongs to $C_{0}$. Hence the horosphere centered at $\xi$ passing through $\pi_{C_{0}}(\xi)$ meets the geodesic ray from $\alpha x_{0}$ to $\xi$ in a point $u$. As $C_{0}$ is convex and $\pi_{C_{0}}(\xi)$ is the closed point in $C_{0}$ to $\xi$, by an easy $\mathrm{CAT}(-1)$ comparison argument, the distance $d\left(u, \pi_{C_{0}}(\xi)\right)$ is at most 1 (and even at most $\log \frac{3+\sqrt{5}}{2}$ ). By the triangle inequality,

$$
\beta_{\xi}\left(\alpha x_{0}, \pi_{C_{0}}(\xi)\right)=d\left(\alpha x_{0}, u\right) \geq d\left(\alpha x_{0}, \pi_{C_{0}}(\xi)\right)-d\left(u, \pi_{C_{0}}(\xi)\right) .
$$

Therefore

$$
\begin{aligned}
\beta_{\xi}\left(\alpha x_{0}, x_{0}\right) & =\beta_{\xi}\left(\alpha x_{0}, \pi_{C_{0}}(\xi)\right)-\beta_{\xi}\left(x_{0}, \pi_{C_{0}}(\xi)\right) \\
& \geq d\left(\alpha x_{0}, \pi_{C_{0}}(\xi)\right)-1-d\left(x_{0}, \pi_{C_{0}}(\xi)\right) \\
& \geq d\left(\alpha x_{0}, x_{0}\right)-1-2 d\left(x_{0}, \pi_{C_{0}}(\xi)\right),
\end{aligned}
$$

where the last inequality is again obtained by the triangle inequality. Hence

$$
\sum_{\alpha \in \Gamma_{0}} \frac{d \mu_{\alpha x_{0}}}{d \mu_{x_{0}}}(\xi)=\sum_{\alpha \in \Gamma_{0}} e^{-\delta \beta_{\xi}\left(\alpha x_{0}, x_{0}\right)} \leq e^{\delta\left(1+2 d\left(x_{0}, \pi_{C_{0}}(\xi)\right)\right)} P_{x_{0}, \Gamma_{0}}(\delta) .
$$

The right hand side, as $\delta>\delta_{0}$, is a positive continuous map of $\xi \in$ $\partial_{\infty} X-\Lambda \Gamma_{0}$. Hence $\widetilde{\mu}_{\Gamma_{0} x_{0}}$ is a locally finite Borel measure on $\partial_{\infty} X-\Lambda \Gamma_{0}$. It is clearly invariant under $\Gamma_{0}$ by construction and the equivariance property of $\left(\mu_{x}\right)_{x \in X}$. As $\beta_{\xi}\left(\alpha x_{0}, x_{0}\right) \leq d\left(\alpha x_{0}, x_{0}\right)$, we have, for every $\xi$ in $\partial_{\infty} X-\Lambda \Gamma_{0}$,

$$
P_{x_{0}, \Gamma_{0}}(\delta) \leq \frac{d \widetilde{\mu}_{\Gamma_{0} x_{0}}}{d \mu_{x_{0}}}(\xi) \leq e^{\delta\left(1+2 d\left(x_{0}, \pi_{C_{0}}(\xi)\right)\right)} P_{x_{0}, \Gamma_{0}}(\delta),
$$

hence $\widetilde{\mu}_{\Gamma_{0} x_{0}}$ and $\mu_{x_{0}}$ have the same measure class on $\partial_{\infty} X-\Lambda \Gamma_{0}$.

q.e.d.

The next result, of independent interest, is a fluctuating density result a la Sullivan, where the parabolic subgroup (as in for instance [HP4]) has been replaced by a convex-cocompact subgroup. It will be used in this paper to check Assumption (5) in Theorem 3.1.

Theorem 4.4. There exist two constants $c \geq 1$ and $c^{\prime}>0$ such that, for every $r$ in $R_{0}$ and $\epsilon$ in $\left.] 0, c^{\prime} e^{-D(r)}\right]$,

$$
\frac{1}{c} e^{-\delta_{0} D(r)} \epsilon^{\delta-\delta_{0}} \leq \mu_{\Gamma_{0} x_{0}}\left(\mathscr{N}_{r}(\epsilon)\right) \leq c e^{-\delta_{0} D(r)} \epsilon^{\delta-\delta_{0}} .
$$

Proof. For every double coset $r$ in $R_{0}$, choose a representative $\gamma_{r}$ of $r$ such that

$$
d\left(x_{0}, \gamma_{r} x_{0}\right)=\min _{\alpha, \alpha^{\prime} \in \Gamma_{0}} d\left(x_{0}, \alpha \gamma_{r} \alpha^{\prime} x_{0}\right) .
$$

Denote by $\mathscr{N}_{\epsilon^{\prime}, d^{\prime}}(A)$ the (closed) $\epsilon^{\prime}$-neighborhood of a given subset $A$ for a distance or a distance-like map $d^{\prime}$. By Lemma 2.3, the subset $\mathscr{N}_{\epsilon, d_{C_{0}}}\left(\gamma_{r} \Lambda \Gamma_{0}\right)$ of $\partial_{\infty} X-\partial_{\infty} C_{0}$ is compact, and its diameter for the distance-like map $d_{C_{0}}$ tends to 0 as $D(r)$ tends to $+\infty$ and $\epsilon$ tends to 0 . 
Recall that $\Gamma_{0}$ acts isometrically and properly with compact quotient on $\partial_{\infty} X-\partial_{\infty} C_{0}$ for the distance-like map $d_{C_{0}}$. Hence there exists $N^{\prime} \in \mathbb{N}$ and $c_{1}^{\prime}>0$ such that for every $\epsilon$ in $\left.] 0, c_{1}^{\prime}\right]$, for every $r$ in $R_{0}$, we have

$$
\operatorname{Card}\left\{\alpha \in \Gamma_{0}: \alpha \mathscr{N}_{\epsilon, d_{C_{0}}}\left(\gamma_{r} \Lambda \Gamma_{0}\right) \cap \mathscr{N}_{\epsilon, d_{C_{0}}}\left(\gamma_{r} \Lambda \Gamma_{0}\right) \neq \emptyset\right\} \leq N^{\prime} .
$$

By the construction of $\mu_{\Gamma_{0} x_{0}}$, we have, for every $r$ in $R_{0}$ and $\epsilon$ in ] $\left.0, c_{1}^{\prime}\right]$

(5)

$$
\frac{1}{N^{\prime}} \widetilde{\mu}_{\Gamma_{0} x_{0}}\left(\mathscr{N}_{\epsilon, d_{C_{0}}}\left(\gamma_{r} \Lambda \Gamma_{0}\right)\right) \leq \mu_{\Gamma_{0} x_{0}}\left(\mathscr{N}_{r}(\epsilon)\right) \leq \widetilde{\mu}_{\Gamma_{0} x_{0}}\left(\mathscr{N}_{\epsilon, d_{C_{0}}}\left(\gamma_{r} \Lambda \Gamma_{0}\right)\right) .
$$

As $\Gamma_{0} \backslash C_{0}$ is compact and by the definition of the representatives $\gamma_{r}$, there exists $c_{2}^{\prime}>0$ such that, for every $r$ in $R_{0}$, for every element $x$ in $\gamma_{r}\left(C_{0} \cup \partial_{\infty} C_{0}\right)$, the closest point to $x$ on $C_{0}$ is at distance at most $c_{2}^{\prime}$ from $x_{0}$ (see also [HP4, Lem. 3.5]).

Hence, there exists a compact subset $K$ of $\partial_{\infty} X-\partial_{\infty} C_{0}$ which contains $\gamma_{r} \Lambda \Gamma_{0}$ for every $r$ in $R_{0}$. By Lemma 2.3 (2), there exists a compact subset $K^{\prime}$ of $\partial_{\infty} X-\partial_{\infty} C_{0}$ which contains $\mathscr{N}_{\epsilon, d_{C_{0}}}\left(\gamma_{r} \Lambda \Gamma_{0}\right)$ for every $\epsilon$ in ]0, $\left.c_{1}^{\prime}\right]$ and every $r$ in $R_{0}$. Hence by Lemma 2.3 (1), there exist two constants $c_{3}^{ \pm}>0$ such that for every $r$ in $R_{0}$ and $\left.\left.\epsilon \in\right] 0, c_{1}^{\prime}\right]$,

$$
\mathscr{N}_{c_{3}^{-} \epsilon, d_{x_{0}}}\left(\gamma_{r} \Lambda \Gamma_{0}\right) \subset \mathscr{N}_{\epsilon, d_{C_{0}}}\left(\gamma_{r} \Lambda \Gamma_{0}\right) \subset \mathscr{N}_{c_{3}^{+} \epsilon, d_{x_{0}}}\left(\gamma_{r} \Lambda \Gamma_{0}\right) .
$$

As $K^{\prime}$ and $\partial_{\infty} C_{0}$ are compact and disjoint, if $c_{1}^{\prime}$ is small enough, then there exists a compact subset $K^{\prime \prime}$ of $\partial_{\infty} X-\partial_{\infty} C_{0}$ containing $\mathscr{N}_{c_{3}^{+} \epsilon, d_{x_{0}}}\left(\gamma_{r} \Lambda \Gamma_{0}\right)$ (and hence $\mathscr{N}_{c_{3}^{-} \epsilon, d_{x_{0}}}\left(\gamma_{r} \Lambda \Gamma_{0}\right)$ ) for every $r$ in $R_{0}$ and $\epsilon$ in $\left.] 0, c_{1}^{\prime}\right]$. By the continuity of $\pi_{C_{0}}$, there exists a constant $c_{4}^{\prime}>0$ such that for every $r$ in $R_{0}$ and $\epsilon$ in $\left.] 0, c_{1}^{\prime}\right]$, the subsets $\pi_{C_{0}}\left(\mathscr{N}_{c_{3}^{ \pm} \epsilon, d_{x_{0}}}\left(\gamma_{r} \Lambda \Gamma_{0}\right)\right)$ are contained in the ball of center $x_{0}$ and radius $c_{4}^{\prime}$.

By the definition of the representatives $\gamma_{r}$, for every $r$ in $R_{0}$, for every $\xi \in \gamma_{r} \partial_{\infty} C_{0}$, the point $\gamma_{r} x_{0}$ is at distance at most a constant from the geodesic between $x_{0}$ and $\xi$ (see also [HP4, Lem. 3.5]). Recall that for every $\eta, \eta^{\prime}$ in $\partial_{\infty} X$, if $d_{x_{0}}\left(\eta, \eta^{\prime}\right) \leq \epsilon^{\prime}$, then the geodesic rays $\left[x_{0}, \eta[\right.$ and $\left[x_{0}, \eta^{\prime}\right.$ [ remain at distance bounded by a universal constant at least during a time $-\log \epsilon^{\prime}$. Hence, if $c^{\prime} \leq c_{1}^{\prime}$ is small enough and $\epsilon \leq c^{\prime} e^{-D(r)}$, then every geodesic ray from $x_{0}$ to a point $\xi$ in $\mathscr{N}_{c_{3}^{ \pm} \epsilon, d_{x_{0}}}\left(\gamma_{r} \Lambda \Gamma_{0}\right)$ passes at distance less than a constant from $\gamma_{r} x_{0}$. This has two consequences.

- First, using the change of base point formula for the visual distances, there exist two constants $c_{5}^{ \pm}>0$ such that for every $\epsilon \leq c^{\prime} e^{-D(r)}$,

$$
\begin{gathered}
\mathscr{N}_{c_{3}^{+} \epsilon, d_{x_{0}}}\left(\gamma_{r} \Lambda \Gamma_{0}\right) \subset \mathscr{N}_{c_{5}^{+} e^{D(r)} \epsilon, d_{\gamma_{r} x_{0}}}\left(\gamma_{r} \Lambda \Gamma_{0}\right) \text { and } \\
\mathscr{N}_{c_{5}^{-} e^{D(r)} \epsilon, d_{\gamma_{r} x_{0}}}\left(\gamma_{r} \Lambda \Gamma_{0}\right) \subset \mathscr{N}_{c_{3}^{-} \epsilon, d_{x_{0}}}\left(\gamma_{r} \Lambda \Gamma_{0}\right) .
\end{gathered}
$$

- Second, for every $\xi$ in $\mathscr{N}_{c_{3}^{ \pm} \epsilon, d_{x_{0}}}\left(\gamma_{r} \Lambda \Gamma_{0}\right)$, the number $\mid \beta_{\xi}\left(x_{0}, \gamma_{r} x_{0}\right)-$ $d\left(x_{0}, \gamma_{r} x_{0}\right) \mid$ is bounded by a constant. Hence there exist constants $c_{6}^{ \pm}>$ 
0 such that for every $r$ in $R_{0}$ and for every $\xi$ in $\mathscr{N}_{c_{3}^{ \pm} \epsilon, d_{x_{0}}}\left(\gamma_{r} \Lambda \Gamma_{0}\right)$,

$$
c_{6}^{-} e^{-\delta D(r)} \leq \frac{d \mu_{x_{0}}}{d \mu_{\gamma_{r} x_{0}}}(\xi) \leq c_{6}^{+} e^{-\delta D(r)} .
$$

By the Radon-Nykodim derivative estimates in Equation (4) and the definition of $c_{4}^{\prime}$, there exist constants $c_{7}^{ \pm}>0$ such that for every $\epsilon$ in ] $\left.0, c_{1}^{\prime}\right]$, every $r$ in $R_{0}$, and every $\xi$ in $\mathscr{N}_{c_{3}^{ \pm} \epsilon, d_{x_{0}}}\left(\gamma_{r} \Lambda \Gamma_{0}\right)$,

$$
c_{7}^{-} \leq \frac{d \widetilde{\mu}_{\Gamma_{0} x_{0}}}{d \mu_{x_{0}}}(\xi) \leq c_{7}^{+} \text {. }
$$

By Sullivan's shadow lemma (see for instance [Rob, Lem. 1.3]), for every constant $c_{8}^{\prime}>0$ big enough, there exist constants $c_{9}^{ \pm}>0$ such that, for every $\gamma$ in $\Gamma$,

$$
c_{9}^{-} e^{-\delta d\left(x_{0}, \gamma x_{0}\right)} \leq \mu_{x_{0}}\left(\mathscr{O}_{x_{0}} B\left(\gamma x_{0}, c_{8}^{\prime}\right)\right) \leq c_{9}^{+} e^{-\delta d\left(x_{0}, \gamma x_{0}\right)} .
$$

For every $t$ in $\mathbb{R}$, define $\Gamma_{0}[t]=\left\{\alpha \in \Gamma_{0}: d\left(x_{0}, \alpha x_{0}\right) \leq t\right\}$. For every $\left.\left.\epsilon^{\prime} \in\right] 0,1\right]$ and $\kappa>0$, define

$$
\begin{gathered}
A_{\epsilon^{\prime}, \kappa}^{+}=\Gamma_{0}\left[-\log \epsilon^{\prime}+\kappa\right]-\Gamma_{0}\left[-\log \epsilon^{\prime}-\kappa\right] \text { and } \\
A_{\epsilon^{\prime}, \kappa}^{-}=\Gamma_{0}\left[-\log \epsilon^{\prime}+2 \kappa\right]-\Gamma_{0}\left[-\log \epsilon^{\prime}+\kappa\right] .
\end{gathered}
$$

Let $\left.\left.\epsilon^{\prime} \in\right] 0,1\right], \eta \in \Lambda \Gamma_{0}$ and $\eta^{\prime} \in \partial_{\infty} X$ be such that $d_{x_{0}}\left(\eta, \eta^{\prime}\right) \leq \epsilon^{\prime}$. Let $u$ be the point of $\left[x_{0}, \eta\right.$ [ at distance $-\log \epsilon^{\prime}$ from $x_{0}$. By the definition of $d_{x_{0}}$ and the properties of the geodesic rays in a $\operatorname{CAT}(-1)$ metric space, there exists a universal constant $c_{8}^{\prime \prime}>0$ such that $\eta^{\prime}$ belongs to $\mathscr{O}_{x_{0}} B\left(u, c_{8}^{\prime \prime}\right)$. Let $c_{10}^{+}>0$ be strictly bigger than the (finite) diameter of $\Gamma_{0} \backslash C_{0}$. Since $\partial_{\infty} C_{0}=\Lambda \Gamma_{0}$ and by convexity, the geodesic ray $\left[x_{0}, \eta\right.$ [ is contained in $C_{0}$. Hence there exists $\alpha$ in $\Gamma_{0}$ such that $d\left(u, \alpha x_{0}\right)<c_{10}^{+}$. Let $c_{8}^{\prime}$ be big enough (at least $c_{8}^{\prime \prime}+c_{10}^{+}$and such that Equation (11) holds). Then, by the triangle inequality, $B\left(u, c_{8}^{\prime \prime}\right)$ is contained in $B\left(\alpha x_{0}, c_{8}^{\prime}\right)$. Note that again by the triangle inequality, $-\log \epsilon^{\prime}-c_{10}^{+}<d\left(x_{0}, \alpha x_{0}\right)<$ $-\log \epsilon^{\prime}+c_{10}^{+}$. Therefore, for every $\epsilon^{\prime}$ in $\left.] 0,1\right]$, we have

$$
\mathscr{N}_{\epsilon^{\prime}, d_{x_{0}}}\left(\Lambda \Gamma_{0}\right) \subset \bigcup_{\alpha \in A_{\epsilon^{\prime}, c_{10}^{+}}^{+}} \mathscr{O}_{x_{0}} B\left(\alpha x_{0}, c_{8}^{\prime}\right) .
$$

As $\Gamma_{0}$ is convex-cocompact, there exists a constant $c_{8}^{\prime \prime \prime}>0$ such that for every $\alpha$ in $\Gamma_{0}$, the segment $\left[x_{0}, \alpha x_{0}\right]$ is at distance at most $c_{8}^{\prime \prime \prime}$ from a geodesic ray starting from $x_{0}$ and contained in $C_{0}$. Let $c_{10}^{-}>0$ be at least $c_{8}^{\prime \prime \prime}+c_{8}^{\prime}$. Let $\left.\left.\epsilon^{\prime} \in\right] 0,1\right], \alpha \in A_{\epsilon^{\prime}, c_{10}^{-}}^{-}$and $\eta^{\prime} \in \mathscr{O}_{x_{0}} B\left(\alpha x_{0}, c_{8}^{\prime}\right)$. Let $v$ be a point on $\left[x_{0}, \eta^{\prime}\left[\right.\right.$ at distance at most $c_{8}^{\prime}$ from $\alpha x_{0}$. Let $\eta \in \partial_{\infty} C_{0}$ and $u \in\left[x_{0}, \eta\right.$ [ be such that $d\left(u, \alpha x_{0}\right) \leq c_{8}^{\prime \prime \prime}$, which exist by the definition of $c_{8}^{\prime \prime \prime}$. Then by the definition of $d_{x_{0}}$ and the triangle inequality, we have

$$
d_{x_{0}}\left(\eta, \eta^{\prime}\right) \leq e^{\frac{1}{2}\left(d(u, v)-d\left(x_{0}, u\right)-d\left(x_{0}, v\right)\right)} \leq e^{c_{8}^{\prime}+c_{8}^{\prime \prime \prime}-d\left(x_{0}, \alpha x_{0}\right)} \leq \epsilon^{\prime},
$$


since $\alpha \in A_{\epsilon^{\prime}, c_{10}^{-}}^{-}$. Therefore, for every $\epsilon^{\prime}$ in $\left.] 0,1\right]$, we have

$$
\bigcup_{\alpha \in A_{\epsilon^{\prime}, c_{10}^{-}}^{-}} \mathscr{O}_{x_{0}} B\left(\alpha x_{0}, c_{8}^{\prime}\right) \subset \mathscr{N}_{\epsilon^{\prime}, d_{x_{0}}}\left(\Lambda \Gamma_{0}\right) .
$$

If $c_{10}^{+}$and then $c_{10}^{-}$are big enough, as $\Gamma_{0}$ is convex-cocompact with critical exponent $\delta_{0}$ (by for instance [Rob] if $\Gamma_{0}$ is non elementary, and even if $\delta_{0}=0$, since then, by the assumptions, $\Gamma_{0}$ contains a hyperbolic element generating a finite index (infinite cyclic) subgroup), there exist constants $c_{11}^{ \pm}>0$ such that for every $\epsilon^{\prime}$ in $\left.] 0,1\right]$, we have

$$
\text { Card } A_{\epsilon^{\prime}, c_{10}^{-}}^{-} \geq c_{11}^{-}\left(\epsilon^{\prime}\right)^{-\delta_{0}} \text { and Card } A_{\epsilon^{\prime}, c_{10}^{+}}^{+} \leq c_{11}^{+}\left(\epsilon^{\prime}\right)^{-\delta_{0}} \text {. }
$$

Let $A_{\epsilon^{\prime}}^{*}$ be a maximal subset of $A_{\epsilon^{\prime}, c_{10}^{-}}^{-}$such that, when $\alpha$ ranges over $A_{\epsilon^{\prime}}^{*}$, the shadows $\mathscr{O}_{x_{0}} B\left(\alpha x_{0}, c_{8}^{\prime}\right)$ are pairwise disjoint. By maximality, for every $\alpha$ in $A_{\epsilon^{\prime}, c_{10}^{-}}^{-}$, there exists $\alpha^{\prime}$ in $A_{\epsilon^{\prime}}^{*}$ such that $\alpha x_{0}$ and $\alpha^{\prime} x_{0}$ are at bounded distance. Hence there exists a constant $c_{12}^{\prime}>0$ such that Card $A_{\epsilon^{\prime}}^{*} \geq c_{12}^{\prime}$ Card $A_{\epsilon^{\prime}, c_{10}^{-}}^{-}$.

Let us now prove the upper bound in Theorem 4.4. Let $c^{\prime}>0$, $r \in R_{0}$ and $\left.\left.\epsilon \in\right] 0, c^{\prime} e^{-D(r)}\right]$. Fix $c^{\prime}$ small enough so that $\epsilon \leq c_{1}^{\prime}$ and $c_{5}^{ \pm} e^{D(r)} \epsilon \leq c_{5}^{ \pm} c^{\prime} \leq 1$. We have

$$
\begin{aligned}
\mu_{\Gamma_{0} x_{0}}\left(\mathscr{N}_{r}(\epsilon)\right) \leq & \widetilde{\mu}_{\Gamma_{0} x_{0}}\left(\mathscr{N}_{\epsilon, d_{C_{0}}}\left(\gamma_{r} \Lambda \Gamma_{0}\right)\right) \leq \widetilde{\mu}_{\Gamma_{0} x_{0}}\left(\mathscr{N}_{c_{3}^{+} \epsilon, d_{x_{0}}}\left(\gamma_{r} \Lambda \Gamma_{0}\right)\right) \\
& \quad \text { by }(5) \text { and }(6), \\
\leq & c_{7}^{+} \mu_{x_{0}}\left(\mathscr{N}_{c_{3}^{+} \epsilon, d_{x_{0}}}\left(\gamma_{r} \Lambda \Gamma_{0}\right)\right) \text { by }(10), \\
\leq & c_{7}^{+} c_{6}^{+} e^{-\delta D(r)} \mu_{\gamma_{r} x_{0}}\left(\mathscr{N}_{c_{5}^{+} e^{D(r)} \epsilon, d_{\gamma_{r} x_{0}}}\left(\gamma_{r} \Lambda \Gamma_{0}\right)\right) \\
& \quad \text { by }(9) \text { and }(7), \\
= & c_{7}^{+} c_{6}^{+} e^{-\delta D(r)} \mu_{x_{0}}\left(\mathscr{N}_{c_{5}^{+} e^{D(r)} \epsilon, d_{x_{0}}}\left(\Lambda \Gamma_{0}\right)\right) \text { by invariance, } \\
\leq & c_{7}^{+} c_{6}^{+} e^{-\delta D(r)} \quad \sum_{\alpha \in A_{c_{5}^{+} e^{D(r)} \epsilon, c_{10}^{+}}} \mu_{x_{0}}\left(\mathscr{O}_{x_{0}} B\left(\alpha x_{0}, c_{8}^{\prime}\right)\right) \\
& \quad \operatorname{by}(12) \text { with } \epsilon^{\prime}=c_{5}^{+} e^{D(r)} \epsilon, \\
\leq & c_{7}^{+} c_{6}^{+} e^{-\delta D(r)} c_{9}^{+} e^{-\delta\left(-\log \left(c_{5}^{+} e^{D(r)} \epsilon\right)-c_{10}^{+}\right)} c_{11}^{+}\left(c_{5}^{+} e^{D(r)} \epsilon\right)^{-\delta_{0}} \\
& \quad \operatorname{by}(11) \text { and }(14), \\
= & c_{13}^{+} e^{-\delta_{0} D(r)} \epsilon^{\delta-\delta_{0}},
\end{aligned}
$$

for some constant $c_{13}^{+}>0$, which proves the upper bound.

Similarly for the lower bound, 


$$
\begin{aligned}
& \mu_{\Gamma_{0} x_{0}}\left(\mathscr{N}_{r}(\epsilon)\right) \\
& \geq \frac{1}{N^{\prime}} \widetilde{\mu}_{\Gamma_{0} x_{0}}\left(\mathscr{N}_{\epsilon, d_{C_{0}}}\left(\gamma_{r} \Lambda \Gamma_{0}\right)\right) \geq \frac{1}{N^{\prime}} \widetilde{\mu}_{\Gamma_{0} x_{0}}\left(\mathscr{N}_{c_{3}^{-} \epsilon, d_{x_{0}}}\left(\gamma_{r} \Lambda \Gamma_{0}\right)\right) \\
& \geq \frac{c_{7}^{-} c_{6}^{-}}{N^{\prime}} e^{-\delta D(r)} \mu_{x_{0}}\left(\mathscr{N}_{c_{5}^{-} e^{D(r)} \epsilon, d_{x_{0}}}\left(\Lambda \Gamma_{0}\right)\right) \\
& \geq \frac{c_{7}^{-} c_{6}^{-}}{N^{\prime}} e^{-\delta D(r)} \mu_{x_{0}}\left(\bigcup_{\alpha \in A_{c_{5}^{*} e^{-D(r)} \epsilon}} \mathscr{O}_{x_{0}} B\left(\alpha x_{0}, c_{8}^{\prime}\right)\right) \\
& =\frac{c_{7}^{-} c_{6}^{-}}{N^{\prime}} e^{-\delta D(r)} \sum_{\alpha \in A_{c_{5}^{*} e^{D}(r) \epsilon}^{*}} \mu_{x_{0}}\left(\mathscr{O}_{x_{0}} B\left(\alpha x_{0}, c_{8}^{\prime}\right)\right) \\
& \geq \frac{c_{7}^{-} c_{6}^{-}}{N^{\prime}} e^{-\delta D(r)} c_{9}^{-} e^{-\delta\left(-\log \left(c_{5}^{-} e^{D(r)} \epsilon\right)+2 c_{10}^{-}\right)} c_{12}^{\prime} c_{11}^{-}\left(c_{5}^{-} e^{D(r)} \epsilon\right)^{-\delta_{0}} \\
& =c_{13}^{-} e^{-\delta_{0} D(r)} \epsilon^{\delta-\delta_{0}} \text {, }
\end{aligned}
$$

for some constant $c_{13}^{-}>0$, which proves the result.

q.e.d.

Lemma 4.5. For every $N \in \mathbb{N}-\{0\}$, there exists $c^{\prime \prime}>0$ such that for every $n$ in $\mathbb{N}$, for every distinct $r$ and $r^{\prime}$ in $R_{0}$ such that $D(r)$ and $D\left(r^{\prime}\right)$ belong to $\left[n N,(n+1) N\left[\right.\right.$, the subsets $\mathscr{N}_{r}\left(c^{\prime \prime} e^{-n N}\right)$ and $\mathscr{N}_{r^{\prime}}\left(c^{\prime \prime} e^{-n N}\right)$ are disjoint.

Proof. Let $N \in \mathbb{N}-\{0\}$, and $c^{\prime \prime} \leq 1$ be small enough, to be determined during the proof. Assume by absurd that there exists $n$ in $\mathbb{N}$, distinct $r$ and $r^{\prime}$ in $R_{0}$ such that $D(r), D\left(r^{\prime}\right) \in[n N,(n+1) N[$, and that the subsets $\mathscr{N}_{r}\left(c^{\prime \prime} e^{-n N}\right)$ and $\mathscr{N}_{r^{\prime}}\left(c^{\prime \prime} e^{-n N}\right)$ have non empty intersection. Then, there exist representatives $\gamma, \gamma^{\prime}$ of the double cosets $r, r^{\prime}$ and points $\xi, \xi^{\prime}$ in $\gamma \Lambda \Gamma_{0}, \gamma^{\prime} \Lambda \Gamma_{0}$ respectively, and an element $\eta$ in $\partial_{\infty} X-\partial_{\infty} C_{0}$ which is different from $\xi, \xi^{\prime}$, such that $d_{C_{0}}(\xi, \eta)$ and $d_{C_{0}}\left(\xi^{\prime}, \eta\right)$ are at most $c^{\prime \prime} e^{-n N}$, and in particular at most $c^{\prime \prime}$.

Since there are only finitely many $r$ 's with $D(r)$ less than a constant (by Lemma 4.1), and since the subsets $\gamma \Lambda \Gamma_{0}$ for $\gamma$ in $\left(\Gamma-\Gamma_{0}\right) / \Gamma_{0}$ are pairwise disjoint (by Proposition $2.6(2)$ ) closed subsets, we may assume that $D(r)$ and $D\left(r^{\prime}\right)$ are bigger than any given constant $c_{1}^{\prime \prime}>0$. In particular, $D(r)$ and $D\left(r^{\prime}\right)$ are positive.

By Lemma $2.3(4)$, there exists a universal constant $c_{2}^{\prime \prime} \geq 1$ such that if $d_{C_{0}}\left(\eta^{\prime}, \eta^{\prime \prime}\right) \leq 1 / c_{2}^{\prime \prime}$, then the geodesic line between $\eta^{\prime}$ and $\eta^{\prime \prime}$ is disjoint from $C_{0}$, and the length of the shortest segment between $] \eta^{\prime}, \eta^{\prime \prime}[$ and $C_{0}$ is at most $-\log d_{C_{0}}\left(\eta^{\prime}, \eta^{\prime \prime}\right)+c_{2}^{\prime \prime}$ and at least $-\log d_{C_{0}}\left(\eta^{\prime}, \eta^{\prime \prime}\right)-c_{2}^{\prime \prime}$. Assume that $c^{\prime \prime} \leq 1 / c_{2}^{\prime \prime}$. 


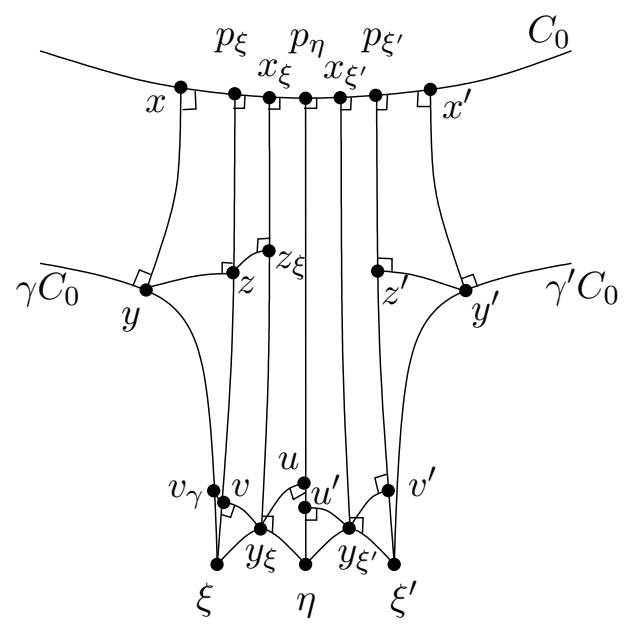

Let $p_{\xi}, p_{\xi^{\prime}}, p_{\eta}$ be the closest point on $C_{0}$ to $\xi, \xi^{\prime}, \eta$ respectively. Let $[x, y]$ (resp. $\left[x^{\prime}, y^{\prime}\right] ;\left[x_{\xi}, y_{\xi}\right] ;\left[x_{\xi^{\prime}}, y_{\xi^{\prime}}\right]$ ) be the shortest segment between $C_{0}$ and $\gamma C_{0}$ (resp. $C_{0}$ and $\gamma^{\prime} C_{0} ; C_{0}$ and $] \xi, \eta\left[; C_{0}\right.$ and $] \xi^{\prime}, \eta[$ ), with $x, x^{\prime}, x_{\xi}, x_{\xi^{\prime}}$ in $C_{0}$. Let $z, z^{\prime}$ be the closest point to $y, y^{\prime}$ on $\left[p_{\xi}, \xi\left[,\left[p_{\xi^{\prime}}, \xi^{\prime}[\right.\right.\right.$ respectively. Let $v, u, v^{\prime}, u^{\prime}$ be the closest points to $y_{\xi}, y_{\xi}, y_{\xi^{\prime}}, y_{\xi^{\prime}}$ on $\left[p_{\xi}, \xi\left[,\left[p_{\eta}, \eta\left[,\left[p_{\xi^{\prime}}, \xi^{\prime}\left[,\left[p_{\eta}, \eta[\right.\right.\right.\right.\right.\right.\right.$ respectively (see the above picture). We have $d(x, y)=D(r), d\left(x^{\prime}, y^{\prime}\right)=D\left(r^{\prime}\right)$.

Assume that $c^{\prime \prime} \leq e^{-c_{1}^{\prime \prime}-c_{2}^{\prime \prime}}$, so that $d\left(x_{\xi}, y_{\xi}\right)$ is at least $-\log \left(c^{\prime \prime}\right)-c_{2}^{\prime \prime} \geq$ $c_{1}^{\prime \prime}$. By the convexity of $C_{0}$ and quasi-geodesic arguments, if $c_{1}^{\prime \prime}$ is bigger than some universal constant, then there exists a universal constant $c_{3}^{\prime \prime} \geq$ 0 such that the distances $d(z, y), d\left(x, p_{\xi}\right), d\left(z^{\prime}, y^{\prime}\right), d\left(x^{\prime}, p_{\xi^{\prime}}\right), d\left(v, y_{\xi}\right)$, $d\left(u, y_{\xi}\right), d\left(v^{\prime}, y_{\xi^{\prime}}\right), d\left(u^{\prime}, y_{\xi^{\prime}}\right), d\left(p_{\xi}, x_{\xi}\right), d\left(p_{\eta}, x_{\xi}\right), d\left(p_{\eta}, x_{\xi^{\prime}}\right), d\left(p_{\xi^{\prime}}, x_{\xi^{\prime}}\right)$ are at most $c_{3}^{\prime \prime}$. By convexity of $C_{0}$, the point $x_{\xi}$ is the closest point to $p_{\xi}$ on $\left[x_{\xi}, y_{\xi}\right]$. Since the closest point maps do not increase distances, we have $d\left(p_{\xi}, z\right) \leq d(x, y)$, and similarly $d\left(p_{\xi^{\prime}}, z^{\prime}\right) \leq d\left(x^{\prime}, y^{\prime}\right)$. Hence

$$
\begin{aligned}
d(v, z) & \geq d\left(v, p_{\xi}\right)-d\left(p_{\xi}, z\right) \geq d\left(x_{\xi}, y_{\xi}\right)-2 c_{3}^{\prime \prime}-d(x, y) \\
& \geq\left(-\log \left(c^{\prime \prime} e^{-n N}\right)-c_{2}^{\prime \prime}\right)-2 c_{3}^{\prime \prime}-N(n+1) \\
& =-\log c^{\prime \prime}-N-c_{2}^{\prime \prime}-2 c_{3}^{\prime \prime} .
\end{aligned}
$$

If $c^{\prime \prime}$ is small, this implies that the points $p_{\xi}, z, v$ are in this order on $\left[p_{\xi}, \xi\left[\right.\right.$, and, with $v_{\gamma}$ the closest point to $v$ on $\left[y, \xi\left[\right.\right.$, that $d\left(y, v_{\gamma}\right) \geq$ $d(z, v)-c_{3}^{\prime \prime} \geq-\log c^{\prime \prime}-N-c_{2}^{\prime \prime}-3 c_{3}^{\prime \prime}$ is big.

Up to permuting $\xi$ and $\xi^{\prime}$, we may assume that $p_{\eta}, u, u^{\prime}, \eta$ are in this order on the geodesic ray $\left[p_{\eta}, \eta[\right.$. By the convexity properties of the distance, since $d\left(u^{\prime}, v^{\prime}\right) \leq 2 c_{3}^{\prime \prime}$ and $d\left(p_{\eta}, p_{\xi}\right) \leq 2 c_{3}^{\prime \prime}$, we have, with $w$ the closest point to $u$ on $\left[p_{\xi^{\prime}}, \xi^{\prime}\left[\right.\right.$, that $d(u, w) \leq 2 c_{3}^{\prime \prime}$. Hence, since 


$$
\begin{aligned}
d\left(x^{\prime}, y^{\prime}\right) & \leq N(n+1) \leq d(x, y)+N, \text { we have } \\
d\left(w, z^{\prime}\right) & \geq d\left(w, p_{\xi^{\prime}}\right)-d\left(p_{\xi^{\prime}}, z^{\prime}\right) \\
& \geq\left(-d\left(w, y_{\xi}\right)+d\left(y_{\xi}, x_{\xi}\right)-d\left(x_{\xi}, p_{\xi^{\prime}}\right)\right)-d\left(x^{\prime}, y^{\prime}\right) \\
& \geq d\left(y_{\xi}, x_{\xi}\right)-d(x, y)-6 c_{3}^{\prime \prime}-N \geq-\log c^{\prime \prime}-2 N-c_{2}^{\prime \prime}-6 c_{3}^{\prime \prime} .
\end{aligned}
$$

If $c^{\prime \prime}$ is small, this implies that the points $p_{\xi^{\prime}}, z^{\prime}, w$ are in this order on $\left[p_{\xi^{\prime}}, \xi^{\prime}\left[\right.\right.$, so that the point $w^{\prime}$ in $\left[y^{\prime}, \xi^{\prime}\left[\right.\right.$, whose closest point on $\left[z^{\prime}, \xi^{\prime}\right]$ is $w$, exists and satisfies $d\left(w, w^{\prime}\right) \leq d\left(y^{\prime}, z^{\prime}\right) \leq c_{3}^{\prime \prime}$ by convexity, and that $d\left(y^{\prime}, w^{\prime}\right) \geq d\left(z^{\prime}, w\right) \geq-\log c^{\prime \prime}-2 N-c_{2}^{\prime \prime}-6 c_{3}^{\prime \prime}$ is big.

In the geodesic quadrilateral with vertices $p_{\xi}, p_{\xi^{\prime}}, v$ and $w$, we have $d\left(p_{\xi}, p_{\xi^{\prime}}\right) \leq 4 c_{3}^{\prime \prime}$ and $d(v, w) \leq 4 c_{3}^{\prime \prime}$. Hence, by convexity, $z^{\prime}$ is at distance at most $4 c_{3}^{\prime \prime}$ from $\left[p_{\xi}, v\right]$, and by the triangular inequality,

$$
d\left(z, z^{\prime}\right) \leq\left|d\left(p_{\xi}, z\right)-d\left(p_{\xi^{\prime}}, z^{\prime}\right)\right|+8 c_{3}^{\prime \prime} .
$$

Since $\left|d\left(p_{\xi}, z\right)-d(x, y)\right| \leq 2 c_{3}^{\prime \prime},\left|d\left(p_{\xi^{\prime}}, z^{\prime}\right)-d\left(x^{\prime}, y^{\prime}\right)\right| \leq 2 c_{3}^{\prime \prime}$, and $\mid d(x, y)-$ $d\left(x^{\prime}, y^{\prime}\right) \mid \leq N$, we then have $d\left(z, z^{\prime}\right) \leq 12 c_{3}^{\prime \prime}+N$. Therefore

$$
d\left(y, y^{\prime}\right) \leq d(y, z)+d\left(z, z^{\prime}\right)+d\left(z^{\prime}, y^{\prime}\right) \leq 14 c_{3}^{\prime \prime}+N .
$$

Now, the geodesic segments $\left[y, v_{\gamma}\right]$ and $\left[y^{\prime}, w^{\prime}\right]$, contained respectively in $\gamma C_{0}$ and $\gamma^{\prime} C_{0}$, are arbitrarily long if $c^{\prime \prime}$ is small enough. Moreover their first endpoints $y, y^{\prime}$ and last endpoints $v_{\gamma}, w$ are at bounded distance, by Equation (15) and since $d\left(v_{\gamma}, w^{\prime}\right) \leq 6 c_{3}^{\prime \prime}$. Let $\epsilon=1$ and $\kappa(\epsilon)$ be given by Proposition 2.6 (4). Hence by hyperbolicity, the $\epsilon$ neighborhoods of $\gamma C_{0}$ and $\gamma^{\prime} C_{0}$ meet in a segment of length that can be made bigger than $\kappa(\epsilon)$ if $c^{\prime \prime}$ is small enough. This is a contradiction to Proposition 2.6 (4).

q.e.d.

A map $\psi:[0,+\infty[\rightarrow] 0,+\infty[$ is called slowly varying (see $[\mathbf{S u l}])$ if it is measurable and if there exist constants $B>0$ and $A \geq 1$ such that for every $x, y$ in $\mathbb{R}_{+}$, if $|x-y| \leq B$, then $\psi(y) \leq A \psi(x)$. Recall (see for instance $[\mathbf{H P 4}$, Sec. 5]) that this implies that $\psi$ is locally bounded, hence it is locally integrable; also, if $\log \psi$ is Lipschitz, then $\psi$ is slowly varying; and for every $N \in \mathbb{N}-\{0\}$ and $\epsilon>0$, the series $\sum_{n=0}^{\infty} \psi(N n)^{\epsilon}$ converges if and only if the integral $\int_{0}^{\infty} \psi(t)^{\epsilon} d t$ converges.

The following statement, which could also have other applications, is the main step towards our Khintchine-type theorem for the spiraling of geodesic rays in $\Gamma \backslash X$ around $\Gamma_{0} \backslash C_{0}$. It gives a $0-1$ measure result for the approximation of points in the limit set of $\Gamma$ by points of the orbit under $\Gamma$ of the limit set of $\Gamma_{0}$, and seems to be the first such result when $\Gamma_{0}$ is non elementary.

Theorem 4.6. Let $X, \Gamma, \Gamma_{0}, \delta, \delta_{0},\left(\mu_{x}\right)_{x \in X}$ be as above. Assume furthermore, for some (hence any) $x$ in $X$, that $\mu_{x}$ is ergodic for the action of $\Gamma$, and that Card $\Gamma x \cap B(x, n) \asymp e^{\delta n}$. Let $f:[0,+\infty[\rightarrow] 0,+\infty[$ be a slowly varying map. 
If the integral $\int_{1}^{+\infty} f(t)^{\delta-\delta_{0}} d t$ converges (resp. diverges), then $\mu_{\Gamma_{0} x_{0}}$ almost no (resp. every) point of $\partial_{\infty} X_{0}$ belongs to infinitely many subsets $\mathscr{N}_{r}\left(f(D(r)) e^{-D(r)}\right)$ where $r \in R_{0}$.

Remark. In addition to the hypotheses on $X$ in this theorem, assume in this remark that $X$ is a Riemannian manifold with constant sectional curvature -1 , that $\Gamma$ is convex-cocompact and that $\Gamma_{0}$ is the stabilizer of a geodesic line. Then up to some rewriting, this result is already known, see for instance $[\mathbf{D M P V}]$ or the recent $[\mathbf{B V}]$. But even in this particular case, our techniques are very different from the ones of [DMPV, BV].

Proof. By a similar reduction as in [HP4, Lem. 5.2], we may assume that $f \leq 1$. Define $g=-\log f:[0,+\infty[\rightarrow[0,+\infty[$.

We apply Theorem 3.1 with $Z=\partial_{\infty} X_{0}, \mu=\mu_{\Gamma_{0} x_{0}}, I=R_{0}$, and, for every $r$ in $R_{0}, n \in \mathbb{N}$ and $\epsilon>0$, with $B_{r}(\epsilon)=\mathscr{N}_{r}(\epsilon), I_{n}=\{r \in$ $\left.R_{0}: N n \leq D(r)<N(n+1)\right\}$ where $N$ is as in Proposition 4.2, and $n_{r}=E\left[\frac{D(r)}{N}\right]$ where $E$ denotes the integer part. Define, for every $n$ in $\mathbb{N}$ and $\epsilon>0$,

$$
\begin{gathered}
f_{1}(n)=e^{\delta n N}, \quad f_{2}(n)=c_{2} e^{-n N}, \quad f_{3}(n)=c_{2} e^{-(n N+g(n N))}, \\
f_{4}(n)=c_{2}^{\delta_{0}-\delta} e^{-\delta_{0} n N}, \quad f_{5}(\epsilon)=\epsilon^{\delta-\delta_{0}},
\end{gathered}
$$

where $c_{2}$ is a small enough positive constant. In particular, we assume that $c_{2}$ is less than $c^{\prime} e^{-N}$, where $c^{\prime}$ is the constant defined in Theorem 4.4, and less than the constant $c^{\prime \prime}$ defined in Lemma 4.5. Note that

$$
f_{1}(n) f_{4}(n) f_{5}\left(f_{3}(n)\right)=e^{-\left(\delta-\delta_{0}\right) g(N n)}=f(N n)^{\delta-\delta_{0}} .
$$

Hence, as $f$ is slowly varying, the series $\sum_{n \in \mathbb{N}} f_{1}(n) f_{4}(n) f_{5}\left(f_{3}(n)\right)$ converges if and only if the integral $\int_{1}^{+\infty} f^{\delta-\delta_{0}}$ converges.

Note that $B_{r}(\epsilon)$ is measurable and non-decreasing in $\epsilon$, and that $I_{n}$ is finite by Lemma 4.1. Assumption (1) of Theorem 3.1 is satisfied since $g$ is non negative. The assumptions (2) and (3) are easily verified. Assumption (4) follows from Proposition 4.2. Assumption (5) follows from Theorem 4.4 and the first assumption on $c_{2}$. Assumption (6) is satisfied by Lemma 4.5 and the second assumption on $c_{2}$. Let us check that Assumption (7) of Theorem 3.1 is also satisfied.

Let $r, r^{\prime} \in R_{0}$ with $n=n_{r}<m=n_{r^{\prime}}$ such that $\mathscr{N}_{r}\left(f_{3}(n)\right)$ and $\mathscr{N}_{r^{\prime}}\left(f_{3}(m)\right)$ meet. Hence, there exists two representatives $\gamma, \gamma^{\prime}$ of $r, r^{\prime}$ and two points $\xi, \xi^{\prime}$ in $\gamma \Lambda \Gamma_{0}, \gamma^{\prime} \Lambda \Gamma_{0}$ respectively, as well as a point $\eta$ in $\partial_{\infty} X-\Lambda \Gamma_{0}$, such that $d_{C_{0}}(\xi, \eta) \leq f_{3}(n)$ and $d_{C_{0}}\left(\xi^{\prime}, \eta\right) \leq f_{3}(m)$. Let us prove that there exists a big enough constant $\lambda>0$ such that $\mathscr{N}_{r^{\prime}}\left(f_{2}(m)\right)$ is contained in $\mathscr{N}_{r}\left(\lambda f_{3}(n)\right)$.

Recall that there are only finitely many $r$ 's with $D(r)$ less than a constant. As $d_{0}$ (defined in Equation (3)) is bounded on $\partial_{\infty} X_{0} \times \partial_{\infty} X_{0}$, the $\epsilon$-neighborhood for $d_{0}$ of any non-empty set covers $\partial_{\infty} X_{0}$ if $\epsilon$ is big 
enough. Hence we may assume, if $\lambda$ is big enough, that $D(r)$ and $D\left(r^{\prime}\right)$ are bigger than any given constant $c_{1}>0$. In particular, $D(r)$ and $D\left(r^{\prime}\right)$ are positive.

Let $p_{\xi}, p_{\xi^{\prime}}, p_{\eta}, x, y, x^{\prime}, y^{\prime}, x_{\xi}, y_{\xi}, x_{\xi^{\prime}}, y_{\xi^{\prime}}, z, z^{\prime}, v, u, v^{\prime}, u^{\prime}, v_{\gamma}$ be as in the proof of Lemma 4.5 and its picture. Let $v_{\gamma^{\prime}}$ be the closest point to $v^{\prime}$ on $\left[y^{\prime}, \xi^{\prime}[\right.$.

As in the proof of Lemma 4.5, if $c_{1}$ is bigger than a universal constant and if $c_{2}$ is small enough, then there exists a universal constant $c_{3}$ such that the following distances $d(z, y), d\left(x, p_{\xi}\right), d\left(z^{\prime}, y^{\prime}\right), d\left(x^{\prime}, p_{\xi^{\prime}}\right), d\left(v, y_{\xi}\right)$, $d\left(u, y_{\xi}\right), d\left(v^{\prime}, y_{\xi^{\prime}}\right), d\left(u^{\prime}, y_{\xi^{\prime}}\right), d\left(p_{\xi}, x_{\xi}\right), d\left(p_{\eta}, x_{\xi}\right), d\left(p_{\eta}, x_{\xi^{\prime}}\right), d\left(p_{\xi^{\prime}}, x_{\xi^{\prime}}\right)$, $d\left(v, v_{\gamma}\right), d\left(v^{\prime}, v_{\gamma^{\prime}}\right)$ are at most $c_{3}$. Furthermore, $p_{\xi}, z, v, \xi$ are in this order on $\left[p_{\xi}, \xi\left[\right.\right.$ and similarly $p_{\xi^{\prime}}, z^{\prime}, v^{\prime}, \xi^{\prime}$ are in this order on $\left[p_{\xi^{\prime}}, \xi^{\prime}[\right.$, and $d\left(y, v_{\gamma}\right), d\left(y^{\prime}, v_{\gamma^{\prime}}\right)$ may be taken bigger than any given constant if $c_{2}$ is small enough.

Say that a point $p$ is above $q$ (resp. below $q$ by at most some constant $h>0)$ with respect to $C_{0}$ if $d\left(p, C_{0}\right) \geq d\left(q, C_{0}\right)$ (resp. $d\left(q, C_{0}\right) \geq$ $\left.d\left(p, C_{0}\right) \geq d\left(q, C_{0}\right)-h\right)$. As $m>n$, the point $y^{\prime}$ is above $y$ or below $y$ by at most some universal constant. If the point $y^{\prime}$ was below $u$ by more than some big constant, then, if $c_{2}$ is small enough, some long subsegment of $\left[y^{\prime}, v_{\gamma^{\prime}}\right]$ would have its endpoints at distance at most a few $c_{3}$ 's from the endpoints of some subsegment of $\left[y, v_{\gamma}\right]$, and as in the end of the proof of Lemma 4.5, this would contradict Proposition 2.6 (4). Therefore the point $y^{\prime}$ is either above, or below only by a some constant, the point $u$ and hence $y_{\xi}$. So that for every $\lambda^{\prime \prime}>0$, there exists $\lambda^{\prime}>0$ such that the shadow (seen from $p_{\xi}$ ) of the ball of center $y_{\xi}$ and radius $\lambda^{\prime}>0$ contains the shadow of the ball of center $y^{\prime}$ and radius $\lambda^{\prime \prime}>0$. Note that if $\lambda^{\prime \prime}$ is big enough and if $c_{2}$ is small enough, then the shadow of $B\left(y^{\prime}, \lambda^{\prime \prime}\right)$ contains $\mathscr{N}_{r^{\prime}}\left(f_{2}(m)\right)$, as seen in the proof of Lemma 4.5. But if $\lambda$ is big enough, then $\mathscr{N}_{r}\left(\lambda f_{3}(n)\right)$ contains the shadow of $B\left(y_{\xi}, \lambda^{\prime}\right)$. Hence Assumption (7) of Theorem 3.1 follows.

Let $E_{f}$ be the set of points of $\partial_{\infty} X_{0}$ which belong to infinitely many

$$
B_{r}\left(f_{3}\left(n_{r}\right)\right)=\mathscr{N}_{r}\left(c_{2} e^{-N E\left[\frac{D(r)}{N}\right]} f\left(N E\left[\frac{D(r)}{N}\right]\right)\right)
$$

for $r$ in $R_{0}$, and similarly let $E_{f}^{\prime}$ be the set of points of $\partial_{\infty} X_{0}$ which belong to infinitely many $\mathscr{N}_{r}\left(f(D(r)) e^{-D(r)}\right)$. As $f$ is slowly varying, there exists a constant $c_{4} \geq 1$ such that $E_{\frac{1}{c_{4}} f} \subset E_{f}^{\prime} \subset E_{c_{4} f}$. Hence in order to prove Theorem 4.6, we only have to prove that if $\int_{1}^{+\infty} f^{\delta-\delta_{0}}$ converges (resp. diverges), then $\mu_{\Gamma_{0} x_{0}}\left(E_{f}\right)=0$ (resp. $\left.\mu_{\Gamma_{0} x_{0}}\left({ }^{c} E_{f}\right)=0\right)$. If this integral converges, then the result follows from Part [A] of Theorem 3.1 .

In the divergence case, Part [B] of Theorem 3.1 implies that $\mu_{\Gamma_{0} x_{0}}\left(E_{f}\right)$ is positive. Using the ergodicity of $\mu_{x}$ in a similar way to the end of 
the proof of Theorem 5.1 of $[\mathbf{H P 4}]$, and the fact that $\mu_{x}\left(\Lambda \Gamma_{0}\right)=0$ as $\delta_{0}<\delta$, it follows that $E_{f}$ has full measure.

\section{Spiraling geodesics}

Let us now proceed towards our main result, Theorem 5.3, which is a geometric version of Theorem 4.6, and its first applications. We keep the notation $X, \Gamma, \Gamma_{0}, \delta, \delta_{0}, C_{0},\left(\mu_{x}\right)_{x \in X}$ of the previous section.

Let $\epsilon$ be a positive real number and let $g:[0,+\infty[\rightarrow[0,+\infty[$ be a map such that $t \mapsto f(t)=e^{-g(t)}$ is slowly varying. A geodesic line $\ell$ in $X$ will be called $(\epsilon, g)$-Liouville with respect to $\left(\Gamma, \Gamma_{0}\right)$ if there exist a sequence $\left(t_{n}\right)_{n \in \mathbb{N}}$ of positive times converging to $+\infty$ and a sequence $\left(\gamma_{n}\right)_{n \in \mathbb{N}}$ of elements of $\Gamma$ such that $\ell(t)$ belongs to $\mathscr{N}_{\epsilon}\left(\gamma_{n} C_{0}\right)$ for every $t$ in $\left[t_{n}, t_{n}+g\left(t_{n}\right)\right]$. The following remark implies that up to changing $g$ by an additive constant (or equivalently up to changing $f$ by a multiplicative constant), being $(\epsilon, g)$-Liouville does not depend on $\epsilon$, and depends only on the asymptotic class of $\ell$. We emphasize that the subsets $\mathscr{N}_{\epsilon}\left(\gamma C_{0}\right)$ as $\gamma$ ranges over $\Gamma$ are not assumed to be pairwise disjoint.

Remark 5.1. (1) Note that if $\epsilon^{\prime} \geq \epsilon$ and $g^{\prime} \leq g$, then a geodesic line which is $(\epsilon, g)$-Liouville is $\left(\epsilon^{\prime}, g^{\prime}\right)$-Liouville.

(2) Note that by the hyperbolicity properties of $X$, for every $\epsilon^{\prime}$ in ] $0, \epsilon]$, there exists a constant $c\left(\epsilon, \epsilon^{\prime}\right) \geq 0$ such that for every convex subset $C$ of $X$ and every geodesic line $\ell$ in $X$, if the length $h$ of the intersection of $\ell$ and $\mathscr{N}_{\epsilon} C$ is at least $c\left(\epsilon, \epsilon^{\prime}\right)$, then the length of the intersection of $\ell$ and $\mathscr{N}_{\epsilon^{\prime}} C$ is at least $h-c\left(\epsilon, \epsilon^{\prime}\right.$ ) (see [PP1] for precise estimates). In particular, if $g \geq c\left(\epsilon, \epsilon^{\prime}\right)$, then a geodesic line which is $(\epsilon, g)$-Liouville is $\left(\epsilon^{\prime}, g-c\left(\epsilon, \epsilon^{\prime}\right)\right)$-Liouville.

(3) Recall that two geodesic lines $\ell, \ell^{\prime}$ in $X$ are asymptotic if $d\left(\ell(t), \ell^{\prime}\right)$ (or equivalently $d\left(\ell^{\prime}(t), \ell\right)$ ) is bounded (or equivalently tends to 0 ) as $t$ tends to $+\infty$. Note that $\ell, \ell^{\prime}$ are asymptotic if and only if their points at infinity $\ell(+\infty), \ell^{\prime}(+\infty)$ are equal.

By the strict convexity of $\epsilon$-neighborhoods of convex subsets of $X$, if $\ell$ is an $(\epsilon, g)$-Liouville geodesic line, and $\ell^{\prime}$ is a geodesic line which is asymptotic to $\ell$, then $\ell^{\prime}$ is $(2 \epsilon, g)$-Liouville, as well as $(\epsilon, g-\eta)$-Liouville for every constant $\eta>0$ such that $g \geq \eta$.

(4) Let $p: X \rightarrow \Gamma \backslash X$ be the canonical projection, and endow $\Gamma \backslash X$ with the quotient distance. If a geodesic line $\ell$ in $X$ is $(\epsilon, g)$-Liouville with respect to $\left(\Gamma, \Gamma_{0}\right)$, then there exists a sequence $\left(t_{n}\right)_{n \in \mathbb{N}}$ of positive times converging to $+\infty$ such that $p \circ \ell(t)$ belongs to $\mathscr{N}_{\epsilon}\left(p\left(C_{0}\right)\right)$ for every $t$ in $\left[t_{n}, t_{n}+g\left(t_{n}\right)\right]$. But the converse is not true in general (for instance if $\Gamma \backslash X$ is compact and $\epsilon$ is its diameter).

The converse is true if $\epsilon$ is small enough, when $p$ induces an injection from $\Gamma_{0} \backslash C_{0}$ into $\Gamma \backslash X$, i.e. when $\gamma C_{0} \cap C_{0}=\emptyset$ for $\gamma \in \Gamma-\Gamma_{0}$. Indeed, 
since $\Gamma_{0}$ is convex-cocompact, there exists $\varepsilon_{0}>0$ such that if $\epsilon \leq \varepsilon_{0}$, we have $\gamma \mathscr{N}_{\epsilon} C_{0} \cap \mathscr{N}_{\epsilon} C_{0}=\emptyset$ for $\gamma \in \Gamma-\Gamma_{0}$. This is for instance the case when $X$ is a manifold, $\Gamma$ acts freely on $X$, and $\Gamma_{0} \backslash C_{0}$ is a totally geodesic embedded submanifold $\left(\mathscr{N}_{\epsilon}\left(\Gamma_{0} \backslash C_{0}\right)\right.$ is then a tubular neighborhood).

When $X$ is a manifold, $\Gamma$ acts freely on $X$, and $C_{0}$ is a geodesic line (even when the closed geodesic $\alpha=\Gamma_{0} \backslash C_{0}$ is not simple), if $g \geq 1$, and if $\epsilon$ is small enough (in particular compared to the angles of $\alpha$ at the self-intersection points), if there exists a sequence $\left(t_{n}\right)_{n \in \mathbb{N}}$ of positive times converging to $+\infty$ such that $p \circ \ell(t)$ belongs to $\mathscr{N}_{\epsilon}\left(p\left(C_{0}\right)\right)$ for every $t \in\left[t_{n}, t_{n}+g\left(t_{n}\right)\right]$, then $\ell$ is $(\epsilon, g-1)$-Liouville with respect to $\left(\Gamma, \Gamma_{0}\right)$.

After these remarks, let $\pi_{0}: X \cup\left(\partial_{\infty} X-\partial_{\infty} C_{0}\right) \rightarrow X_{0} \cup \partial_{\infty} X_{0}$ be the canonical projection. The next lemma shows the relation between the (geometric) Liouville property of a geodesic line and the fact that its point at infinity belongs to a limsup subset considered in Theorem 4.6 .

Lemma 5.2. There exists $c^{\prime \prime \prime}>0$ such that for every geodesic line $\ell$ in $X$ such that $\ell(+\infty) \notin \bigcup_{\gamma \in \Gamma} \gamma \partial_{\infty} C_{0}$,

(1) if $\ell$ is $(\epsilon, g)$-Liouville, then the point $\pi_{0}(\ell(+\infty))$ belongs to infinitely many subsets $\mathscr{N}_{r}\left(c^{\prime \prime \prime} f(D(r)) e^{-D(r)}\right)$ for $r$ in $R_{0}$;

(2) conversely, if the point $\pi_{0}(\ell(+\infty))$ belongs to infinitely many subsets $\mathscr{N}_{r}\left(\frac{1}{c^{\prime \prime \prime}} f(D(r)) e^{-D(r)}\right)$ for $r$ in $R_{0}$, then $\ell$ is $(\epsilon, g)$-Liouville.

Proof. (1) Assume that $\ell$ is $(\epsilon, g)$-Liouville. Up to replacing $\epsilon$ by $2 \epsilon$ and $\ell$ by an asymptotic line, as $\ell(+\infty) \notin \partial_{\infty} C_{0}$ and by Remark 5.1 (3), we may assume that $\ell(0)$ is the closest point in $C_{0}$ to $\ell(+\infty)$.

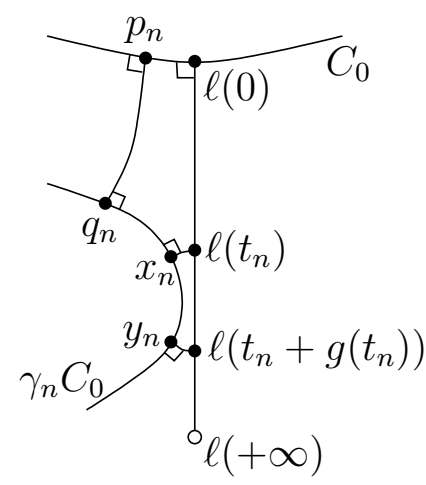

Let $\left(t_{n}\right)_{n \in \mathbb{N}}$ be a sequence of positive times, converging to $+\infty$. Let $\left(\gamma_{n}\right)_{n \in \mathbb{N}}$ in $\Gamma$ be such that $\ell(t) \in \mathscr{N}_{\epsilon}\left(\gamma_{n} C_{0}\right)$ for every $t \in\left[t_{n}, t_{n}+g\left(t_{n}\right)\right]$. As $\Gamma$ acts properly on $X$, and as its subgroup $\Gamma_{0}$ acts cocompactly on $C_{0}$, the family $\left(\gamma C_{0}\right)_{\gamma \in\left(\Gamma-\Gamma_{0}\right) / \Gamma_{0}}$ is locally finite. Hence $d\left(\gamma_{n} C_{0}, C_{0}\right)$ tends to $+\infty$ as $n \rightarrow+\infty$ (otherwise $\ell(+\infty)$ would belong to $\gamma \partial_{\infty} C_{0}$ for some $\gamma \in \Gamma)$. In particular, up to extracting a subsequence, $\gamma_{n} \notin \Gamma_{0}$ 
and with $r_{n}=\left[\gamma_{n}\right] \in R_{0}$, the $r_{n}$ 's are pairwise distinct. Furthermore, we may assume that $\ell$ enters $\mathscr{N}_{\epsilon}\left(\gamma_{n} C_{0}\right)$ at the time $t_{n}$. Let $\left[p_{n}, q_{n}\right]$ be the shortest segment between $C_{0}$ and $\gamma_{n} C_{0}$, with $p_{n} \in C_{0}$, so that $D\left(r_{n}\right)=d\left(p_{n}, q_{n}\right)$. Let $x_{n}$ (resp. $\left.y_{n}\right)$ be a point of $\gamma_{n} C_{0}$ such that $d\left(x_{n}, \ell\left(t_{n}\right)\right) \leq \epsilon\left(\operatorname{resp} . d\left(y_{n}, \ell\left(t_{n}+g\left(t_{n}\right)\right)\right) \leq \epsilon\right)$.

As we have already seen (in the proofs of Theorem 4.4 after Equation (12) and of Lemma 4.5), there exists a constant $c_{1}^{\prime \prime \prime}>0$ such that $\left[q_{n}, y_{n}\right]$ is contained in the $c_{1}^{\prime \prime \prime}$-neighborhood of a geodesic ray $\left[q_{n}, \xi_{n}[\right.$ with $\xi_{n} \in \gamma_{n} \partial_{\infty} C_{0}$, and such that for $n$ big enough, $d\left(p_{n}, \ell(0)\right) \leq c_{1}^{\prime \prime \prime}$. By hyperbolicity, the distance between $p_{n}$ and the closest point of $C_{0}$ to $\xi_{n}$ is at most a constant. By arguments similar to the ones in the proof of Lemma 4.5, it is easy to prove that there exists a constant $c_{2}^{\prime \prime \prime} \geq 0$ such that $-\log d_{C_{0}}\left(\xi_{n}, \ell(+\infty)\right) \geq t_{n}+g\left(t_{n}\right)-c_{2}^{\prime \prime \prime}$ and (using the fact that $\ell$ enters in $\mathscr{N}_{\epsilon}\left(\gamma_{n} C_{0}\right)$ at time $\left.t_{n}\right)$ that $\left|t_{n}-d\left(p_{n}, q_{n}\right)\right| \leq$ $c_{2}^{\prime \prime \prime}$. As $f$ is slowly varying, there exists a constant $c^{\prime \prime \prime} \geq 1$ such that $d_{C_{0}}\left(\xi_{n}, \ell(+\infty)\right) \leq c^{\prime \prime \prime} f\left(D\left(r_{n}\right)\right) e^{-D\left(r_{n}\right)}$. This proves the first assertion.

(2) Assume now that there exist a sequence $\left(r_{n}=\left[\gamma_{n}\right]\right)_{n \in \mathbb{N}}$ of pairwise distinct elements in $R_{0}$ and $\xi_{n} \in \gamma_{n} \partial_{\infty} C_{0}$ such that

$$
d_{C_{0}}\left(\xi_{n}, \ell(+\infty)\right) \leq \frac{1}{c^{\prime \prime \prime}} f\left(D\left(r_{n}\right)\right) e^{-D\left(r_{n}\right)}
$$

for every $n$, for some $c^{\prime \prime \prime} \geq 1$ big enough, to be determined later on. Let us prove that $\ell$ is $(\epsilon, g)$-Liouville. Up to replacing $\epsilon$ by $\frac{\epsilon}{2}$ and $\ell$ by an asymptotic line, we may assume as above that $\ell(0)$ is the closest point in $C_{0}$ to $\ell(+\infty)$.

By Lemma 4.1, we have that $D\left(r_{n}\right)=d\left(C_{0}, \gamma_{n} C_{0}\right)$ tends to $+\infty$ as $n \rightarrow+\infty$ (hence is positive for $n$ big enough). As above, by hyperbolicity, the closest point of $C_{0}$ to $\xi_{n}$ is at distance at most a constant from the closest point of $C_{0}$ to $\gamma_{n} C_{0}$. By hyperbolicity and the definition of $d_{C_{0}}$, there exists a constant $c_{3}^{\prime \prime \prime} \geq 0$ such that between the times $t=D\left(r_{n}\right)$ and $t=D\left(r_{n}\right)+g\left(D\left(r_{n}\right)\right)+\log c^{\prime \prime \prime}-c_{3}^{\prime \prime \prime}$, the geodesic ray $\ell$ is at distance at most $c_{3}^{\prime \prime \prime}$ from $\gamma_{n} C_{0}$. Hence, as in Remark 5.1 (2), there exists a constant $c_{4}^{\prime \prime \prime} \geq 0$ such that both $\ell\left(D\left(r_{n}\right)+c_{4}^{\prime \prime \prime}\right)$ and $\ell\left(D\left(r_{n}\right)+g\left(D\left(r_{n}\right)\right)+\log c^{\prime \prime \prime}-c_{3}^{\prime \prime \prime}-c_{4}^{\prime \prime \prime}\right)$ are at distance at most $\epsilon$ from $\gamma_{n} C_{0}$. Hence if $c^{\prime \prime \prime}$ is big enough, by setting $t_{n}=D\left(r_{n}\right)+c_{4}^{\prime \prime \prime}$ and as $f$ is slowly varying, the second assertion follows.

Theorem 5.3. Let $X$ be a proper $\mathrm{CAT}(-1)$ geodesic metric space. Let $\Gamma$ be a non elementary discrete group of isometries of $X$, with finite critical exponent $\delta$, of divergence type. Let $\widetilde{\mu}_{B M}$ be its Bowen-Margulis measure. Assume that Card $\Gamma x \cap B(x, n) \asymp e^{\delta n}$, for some $x \in X$. Let $\left(\Gamma_{i}\right)_{i \in I}$ be a family of almost malnormal convex-cocompact subgroups of infinite index in $\Gamma$ with critical exponents $\left(\delta_{i}\right)_{i \in I}$. Let $\delta_{0}^{+}=\sup _{i \in I} \delta_{i}$ and $\delta_{0}^{-}=\inf _{i \in I} \delta_{i}$. Let $g:[0,+\infty[\rightarrow[0,+\infty[$ be a map such that $t \mapsto f(t)=e^{-g(t)}$ is slowly varying, and for every $i \in I$, let $\epsilon_{i}>0$. 
If $\int_{1}^{+\infty} f(t)^{\delta-\delta_{0}^{-}} d t$ diverges (resp. $\int_{1}^{+\infty} f(t)^{\delta-\delta_{0}^{+}} d t$ converges), then $\widetilde{\mu}_{B M^{-}}$almost every (resp. no) element of $\mathscr{G} X$ is $\left(\epsilon_{i}, g\right)$-Liouville with respect to $\left(\Gamma, \Gamma_{i}\right)$ for every (resp. some) $i \in I$.

Remark. (1) There are examples of $\Gamma \backslash X$ with $X$ and $\Gamma$ as in the above theorem, such that the upper bound of the critical exponents of the infinite index subgroups in $\Gamma$ is equal to (resp. is strictly less than) the critical exponent of $\Gamma$, as for instance the closed real hyperbolic 3-manifolds fibering over the circle (resp. the closed quaternionic hyperbolic manifolds, see for instance $[\mathbf{L e u}])$.

(2) Assume in this remark that $X$ is a Riemannian manifold, that $\Gamma$ is cocompact and torsion free, and that $\Gamma_{i}$ is the stabilizer of a geodesic line. This corresponds to the hypotheses of Theorem 1.1 (that appear above it). Then there might be a simpler proof using symbolic coding, as indicated to us by V. Kleptsyn, using the fact that the geodesic flow of $\Gamma \backslash X$ is then conjugated to a suspension of a Bernoulli shift. But this requires some serious amount of work, since some geometric features are difficult, to say the least, to translate by the coding. In our general situation, no such coding is possible anyway.

Proof. Note that $\Gamma$ is countable and that any convex-cocompact subgroup of $\Gamma$ is finitely generated. Hence $\Gamma$ contains only countably many convex-cocompact subgroups. So that we may assume that the index set $I$ is countable.

Note that the divergence (resp. convergence) of the integral in the statement is unchanged if one replaces $f$ by a positive scalar multiple of it. Also recall that $\mu_{x_{0}}\left(\gamma \Lambda \Gamma_{i}\right)=0$ for every $i \in I$ and $\gamma \in \Gamma$, since $\delta_{i}<\delta$ (see Lemma 2.5). As $I$ and $\Gamma$ are countable, we have $\mu_{x_{0}}\left(\bigcup_{i \in I, \gamma \in \Gamma} \gamma \Lambda \Gamma_{i}\right)=0$.

When the index set $I$ has only one element, the result follows from Theorem 4.6, by considering the conformal density $\left(\mu_{x}\right)_{x \in X}$ of dimension $\delta$ for $\Gamma$ that is used in the construction (recalled in Section 2) of $\widetilde{\mu}_{\mathrm{BM}}$ (which is ergodic since $\Gamma$ is of divergence type), and by the lemmas 5.2, $4.3,2.4$.

Using the fact that finite or countable unions of sets of measure 0 have measure 0 , the result for general $I$ follows, since

$$
\int_{1}^{+\infty} f^{\delta-\delta_{0}^{-}} \leq \int_{1}^{+\infty} f^{\delta-\delta_{i}} \leq \int_{1}^{+\infty} f^{\delta-\delta_{0}^{+}} \text {. q.e.d. }
$$

Using the three examples at the beginning of Section 4 and Remark 5.1 (4), the theorems 1.1, 1.3, 1.4 in the introduction follow.

Similarly, to prove Proposition 1.5 of the introduction, we apply Theorem 5.3 to $X=T$ the tree in the statement of Proposition 1.5, with $I=\{0\}$ and $\Gamma_{0}$ the stabilizer in $\Gamma$ of a geodesic line in $T$ mapping to 
the cycle $C$ in the statement of Proposition 1.5. For the map $g$ in Theorem 5.3, we take $g / L$ where $g$ is the map in Proposition 1.5 (the map $t \mapsto \exp (-g(t) / L)$ is still slowly varying). When $\Gamma$ is cocompact and torsion-free (anyone of these two assumptions may be not satisfied), then the symbolic dynamics argument alluded to above works easily, and gives an alternative proof. But no such coding is easy in general, even for lattices as simple as $\operatorname{PSL}_{2}\left(\mathbb{F}_{q}[X]\right)$ in $\operatorname{PSL}_{2}\left(\mathbb{F}_{q}\left(\left(X^{-1}\right)\right)\right)$, see for instance [BP].

Many other applications are possible, we will only give the next one.

We refer to $[\mathbf{G P}]$ (see also $[\mathbf{B o u} \mathbf{2}, \mathbf{H a P}]$ ) for the definitions and basic properties of a hyperbolic building, which in particular, when locally finite, is a proper CAT $(-1)$ geodesic metric space. For instance, for every integers $p \geq 5, q \geq 3$, let $\left(W_{p}, S_{p}\right)$ be the hyperbolic Coxeter system generated by the reflections on the sides of a right angled regular real hyperbolic $p$-gon; Bourdon's building $I_{p, q}$ is (see for instance [Bou2]) the unique (up to isomorphism) hyperbolic building of dimension 2, modeled on $\left(W_{p}, S_{p}\right)$, and whose links of vertices are bipartite graphs on $q+q$ vertices. It has a cocompact lattice $\Gamma_{p, q}$ with presentation

$$
\left\langle s_{1}, \ldots, s_{p} \mid \forall i \in \mathbb{Z} / p \mathbb{Z} s_{i}^{q}=1,\left[s_{i}, s_{i+1}\right]=1\right\rangle,
$$

where $s_{1}, \ldots, s_{p}$ are generators of the pointwise stabilizers of the $p$ panels of a fundamental chamber $\mathfrak{C}$ of $I_{p, q}$. If $q$ is even, let $\Gamma_{0}$ be the subgroup (isomorphic to $W_{p}$ ) generated by the elements $s_{i}^{\frac{q}{2}}$ for $1 \leq i \leq p$, which is, by the simple transitivity of the action of $\Gamma_{p, q}$ on the set of chambers, the stabilizer of a (unique) apartment $A_{\mathfrak{C}}$ in $I_{p, q}$ containing $\mathfrak{C}$.

Remark 5.4. If $q$ is even, then the subgroup $\Gamma_{0}$ is almost malnormal in $\Gamma_{p, q}$.

Proof. (F. Haglund) Let $V$ be the union of the closed chambers of $I_{p q}$ meeting $A_{\mathfrak{C}}$, which is invariant by $\Gamma_{0}$. By convexity (and arguments as in Poincaré's theorem about reflection groups), the subgroup $H$ of $\Gamma=\Gamma_{p, q}$ generated by the pointwise stabilizers of the edges contained in the boundary of $V$ has $V$ as a (strict) fundamental domain, and is normalized by $\Gamma_{0}$.

Let $\Gamma^{\prime}$ be the subgroup of $\Gamma$ generated by $H$ and $\Gamma_{0}$, which is isomorphic to their semi-direct product. Since $V$ is a fundamental domain for $H$ and since $\Gamma_{0}$ acts transitively on the chambers of $A_{\mathfrak{C}}$, for every closed chamber $C$ in $A_{\mathfrak{C}}$, for every $g$ in $\Gamma$, there exists $h$ in $H$ and $\gamma_{0}$ in $\Gamma_{0}$ such that $\gamma_{0} h g C$ meets $C$. Since $\Gamma$ is discrete and acts transitively on the chambers, let $\delta_{1}, \ldots, \delta_{k}$ be the elements in $\Gamma$ such that the set of closed chambers meeting $C$ is $\left\{\delta_{1} C, \ldots, \delta_{k} C\right\}$. Since $\Gamma$ acts simply transitively on the chambers, we then have $\Gamma \subset \Gamma^{\prime} \delta_{1} \cup \cdots \cup \Gamma^{\prime} \delta_{k}$, hence $\Gamma^{\prime}$ has finite index in $\Gamma$. 
Let $\Gamma^{\prime \prime}$ be a finite index torsion free subgroup of $\Gamma^{\prime}$ (which exists for instance since $\Gamma_{p, q}$ is finitely generated and linear in characteristic 0 , see for example $[\mathbf{K a p}])$.

Let us prove that the stabilizer $S=\Gamma^{\prime \prime} \cap \Gamma_{0}$ of $A_{\mathfrak{C}}$ in $\Gamma^{\prime \prime}$ is malnormal in $\Gamma^{\prime \prime}$, which proves the result. Assume by absurd that there exists $\gamma$ in $\Gamma^{\prime \prime}-S$ and $s$ in $S-\{e\}$ such that $\gamma s \gamma^{-1}$ preserves $A_{\mathfrak{C}}$. By construction, two distinct translates of $A_{\mathfrak{C}}$ by elements of $\Gamma^{\prime}$ are disjoint. Hence $\gamma^{-1} A_{\mathfrak{C}}$ and $A_{\mathfrak{C}}$ are disjoint, and both preserved by $s$. The (unique) shortest segment between $\gamma^{-1} A_{\mathfrak{C}}$ and $A_{\mathfrak{C}}$ is then fixed by $s$, which contradicts the fact that $\Gamma^{\prime \prime}$ is torsion free.

q.e.d.

Corollary 5.5. Let $X$ be a locally finite thick hyperbolic building modeled on a hyperbolic Coxeter system $(W, S)$. Let $\Gamma$ be a cocompact lattice in the automorphism group of $X$ with Bowen-Margulis measure $\mu$. Let $A$ be an appartment in $X$ whose stabilizer $\Gamma_{A}$ in $\Gamma$ acts cocompactly on $A$ and is almost malnormal in $\Gamma$. Denote by $k \geq 1$ the dimension of $A$ (hence of $X$ ), and by $\delta$ the Hausdorff dimension of $\partial_{\infty} X$ (for any visual distance). Let $f \leq 1$ be a slowly varying map, and $\epsilon>0$.

If $\int_{1}^{+\infty} f(t)^{\delta-k+1} d t$ converges (resp. diverges), then for $\mu$-almost no (resp. every) $\ell$ in $\mathscr{G} X$, there exist a sequence of positive times $\left(t_{n}\right)_{n \in \mathbb{N}}$ converging to $+\infty$ and a sequence $\left(\gamma_{n}\right)_{n \in \mathbb{N}}$ in $\Gamma$ such that $\ell(t)$ belongs to $\gamma_{n} \mathscr{N}_{\epsilon} A$ for every time $t$ in $\left[t_{n}, t_{n}-\log f\left(t_{n}\right)\right]$.

Proof. The apartments in a hyperbolic building are convex (for the CAT(-1) metric), hence $\Gamma_{A}$ is convex-cocompact with critical exponent $k-1$. As $X$ is thick, $\Gamma_{A}$ has infinite index in $\Gamma$. The result follows from Theorem 5.3 (with $I$ a singleton).

q.e.d.

Let us go back to the general situation of Theorem 5.3. The following result is a logarithm law-type result for the spiraling of geodesic lines in $\Gamma \backslash X$ around $\Gamma_{0} \backslash C_{0}$. For every $\epsilon>0$ fixed, define the penetration map $\mathfrak{p}=\mathfrak{p}_{\Gamma \mathscr{N}_{\epsilon} C_{0}}: \mathscr{G} X \times\left[0,+\infty\left[\rightarrow\left[0,+\infty\left[\right.\right.\right.\right.$ in $\Gamma \mathscr{N}_{\epsilon} C_{0}$ of the geodesic lines in $X$, in the following way. For $(\ell, t) \in \mathscr{G} X \times[0,+\infty[$, if $\ell(t)$ does not belong to $\Gamma \mathscr{N}_{\epsilon} C_{0}$, then let $\mathfrak{p}(\ell, t)=0$. Otherwise, let $\mathfrak{p}(\ell, t)$ be the upper bound of the lengths of the intervals $I$ in $\mathbb{R}$ containing $t$ such that there exists $\gamma$ in $\Gamma$ with $\ell(I)$ contained in $\gamma \mathscr{N}_{\epsilon} C_{0}$.

Theorem 5.6. Let $X$ be a proper $C A T(-1)$ geodesic metric space. Let $\Gamma$ be a non elementary discrete group of isometries of $X$, with $f$ nite critical exponent $\delta$, of divergence type, and let $\widetilde{\mu}_{B M}$ be the BowenMargulis measure of $\Gamma$. Assume that Card $\Gamma x \cap B(x, n) \asymp e^{\delta n}$, for some $x \in X$. Let $\Gamma_{0}$ be an almost malnormal convex-cocompact subgroup of infinite index in $\Gamma$ with critical exponent $\delta_{0}$.

Then for every $\epsilon>0$, for $\widetilde{\mu}_{B M}$-almost every $\ell$ in $\mathscr{G} X$, we have

$$
\limsup _{t \rightarrow+\infty} \frac{\mathfrak{p}(\ell, t)}{\log t}=\frac{1}{\delta-\delta_{0}} \text {. }
$$


Proof. For every $\gamma$ in $\Gamma$ such that a geodesic line $\ell$ enters the $\epsilon$ neighborhood of $\gamma C_{0}$, let $t_{\ell, \gamma}$ be the entering time of $\ell$ in this neighborhood.

We apply Theorem 5.3 with $g_{\kappa}: t \mapsto \kappa \log (1+t)$, which is a Lipschitz $\operatorname{map} \mathbb{R}^{+} \rightarrow \mathbb{R}^{+}$, for every $\kappa \geq 0$. Note that the integral $\int_{1}^{+\infty} t^{-\left(\delta-\delta_{0}\right) \kappa} d t$ diverges if and only if $\kappa \leq \frac{1}{\delta-\delta_{0}}$. If $\kappa_{n}=\frac{1}{\delta-\delta_{0}}+\frac{1}{n}$ for $n \in \mathbb{N}-\{0\}$, then the convergence part of Theorem 5.3 implies that for $\widetilde{\mu}_{\mathrm{BM}}$-almost every $\ell$ in $\mathscr{G} X$, for every $\gamma$ in $\Gamma$ such that $\ell$ meets $\gamma \mathscr{N}_{\epsilon} C_{0}$ with $t_{\ell, \gamma}$ big enough, we have $\mathfrak{p}\left(\ell, t_{\ell, \gamma}\right) \leq g_{\kappa_{n}}\left(t_{\ell, \gamma}\right)$. Hence

$$
\limsup _{t \rightarrow+\infty} \frac{\mathfrak{p}(\ell, t)}{\log t}=\limsup \frac{\mathfrak{p}\left(\ell, t_{\ell, \gamma}\right)}{\log \left(1+t_{\ell, \gamma}\right)} \leq \kappa_{n},
$$

where the upper limit is taken on the $\gamma \in \Gamma-\Gamma_{0}$ such that $\ell$ meets $\gamma \mathscr{N}_{\epsilon} C_{0}$ and $t_{\ell, \gamma}$ tends to $+\infty$. As $n \rightarrow+\infty$, we get that $\lim _{\sup _{t \rightarrow+\infty}} \frac{\mathfrak{p}(\ell, t)}{\log t} \leq$ $\frac{1}{\delta-\delta_{0}}$. Similarly, using the divergence part of Theorem 5.3 with the

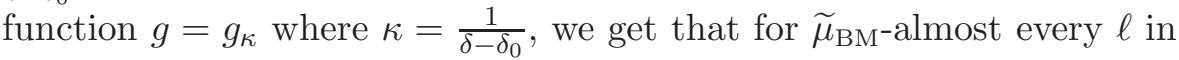
$\mathscr{G} X, \lim \sup _{t \rightarrow+\infty} \frac{\mathfrak{p}(\ell, t)}{\log t} \geq \frac{1}{\delta-\delta_{0}}$. $\quad$ q.e.d.

Corollary 1.2 in the introduction follows immediately.

\section{Non-archimedean Diophantine approximation by quadratic irrational numbers}

Let us now give an application of our results to Diophantine approximation in non-archimedian local fields.

Let $\widehat{K}=\mathbb{F}_{q}\left(\left(X^{-1}\right)\right)$ be the field of formal Laurent series in the variable $X^{-1}$ over the finite field $\mathbb{F}_{q}$. Recall the definition of the absolute value of an element $f \in \widehat{K}-\{0\}$. Let $f=\sum_{i=n}^{\infty} a_{i} X^{-i}$ where $n \in \mathbb{Z}$ and $a_{n} \neq 0$. Then we define $\nu(f)=n$ and $|f|_{\infty}=q^{-\nu(f)}$. Endow the locally compact additive group $\widehat{K}$ with its (unique up to a constant factor) Haar measure $\mu$. Let $K=\mathbb{F}_{q}(X)$.

Let $\mathbb{T}_{q}$ be the Bruhat-Tits tree of $\mathrm{SL}_{2}$ over $\widehat{K}$; we refer to [Ser] for any background on $\mathbb{T}_{q}$. Identify as usual $\partial_{\infty} \mathbb{T}_{q}$ and $\widehat{K} \cup\{\infty\}$, so that the action of $\mathrm{SL}_{2}(\widehat{K})$ on $\mathbb{T}_{q}$ extends continuously by the action by homographies of $\mathrm{SL}_{2}(\widehat{K})$ on $\widehat{K} \cup\{\infty\}$. Let $x_{0}$ be the standard base point in $\mathbb{T}_{q}$. Note that the Hausdorff dimension of the visual distance $d_{x_{0}}$ is $\log q$, as $\mathbb{T}_{q}$ is a regular tree of degree $q+1$.

We refer for instance to [Las, Sch] for nice surveys of the Diophantine approximation properties of elements in $\widehat{K}$ by elements in $K$, a geometric interpretation of which is given in $[\mathbf{P a u}]$. Here, we are interested in approximating elements of $\widehat{K}$ by elements in the set $K_{2}$ of irrational quadratic elements in $\widehat{K}$ over $K$. For every $\alpha$ in $K_{2}$, let $\alpha^{*}$ be its Galois conjugate (the other root of its minimal polynomial), and 
define its height by

$$
h(\alpha)=\left|\alpha-\alpha^{*}\right|_{\infty}^{-1} .
$$

We will not make precise here the (loose) relationship with the standard height (see for instance $[\mathbf{H S}]$ ) of an element of the projective line over the algebraic closure of $K$.

Let $\Gamma=\mathrm{PSL}_{2}\left(\mathbb{F}_{q}[X]\right)=\mathrm{SL}_{2}\left(\mathbb{F}_{q}[X]\right) /\{ \pm \mathrm{id}\}$, which is a (non-uniform) lattice of $\mathbb{T}_{q}$ (see for instance $[$ Ser] $)$, hence a non-elementary discrete group of isometries of $\mathbb{T}_{q}$, whose critical exponent $\delta$ is equal to the Hausdorff dimension of $d_{x_{0}}$, that is $\delta=\log q$. See for instance $[\mathbf{B P}]$ for a (well known) proof that the restrictions to $\partial_{\infty} \mathbb{T}_{q}-\{\infty\}=\widehat{K}$ of the Patterson-Sullivan measures of $\Gamma$ have the same measure class as the Haar measure $\mu$ of $\widetilde{K}$.

Proof of Theorem 1.6. Let $\gamma_{0}$ be a hyperbolic element of $\Gamma, C_{0}$ be its translation axis in $\mathbb{T}_{q}$, and $\Gamma_{0}$ be the stabilizer of $C_{0}$ in $\Gamma$, which is convex-cocompact with critical exponent $\delta_{0}=0$. It is easy to verify that the set of points at infinity of $C_{0}$ is $\left\{\alpha, \alpha^{*}\right\}$ for some $\alpha$ in $K_{2}$; and that any such pair is the set of endpoints of some hyperbolic element of $\Gamma$ (one can for instance use the fact that Artin's continued fraction expansion of an element in $K_{2}$ is eventually periodic (see for example $[$ Las $]))$.

Note that for every $\gamma \in \Gamma$ and $\alpha \in K_{2}$, the element $\gamma \alpha$ is still in $K_{2}$, $(\gamma \alpha)^{*}=\gamma \alpha^{*}$ and $\gamma\left\{\alpha, \alpha^{*}\right\} \cap\left\{\alpha, \alpha^{*}\right\} \neq \emptyset$ if and only if $\gamma \in \Gamma_{0}$.

Denote by $d_{\infty}$ the Hamenstädt distance on $\partial_{\infty} \mathbb{T}_{q}-\{\infty\}=\widehat{K}$ defined by the horosphere centered at $\infty$ and passing through $x_{0}$. It is proved in [Pau, Coro. 5.2] that $d_{\infty}\left(\xi, \xi^{\prime}\right)=\left|\xi-\xi^{\prime}\right|_{\infty}^{\frac{1}{\log q}}$, for every $\xi, \xi^{\prime}$ in $\widehat{K}$.

Lemma 6.1. For every $\xi_{0}$ in $\widehat{K}-\left\{\alpha, \alpha_{*}\right\}$, there exists a neighborhood $V$ of $\xi_{0}$ and a constant $c_{*}>0$ such that for every $\xi, \xi^{\prime}$ in $V$,

$$
d_{C_{0}}\left(\xi, \xi^{\prime}\right)=c_{*} d_{\infty}\left(\xi, \xi^{\prime}\right)=c_{*}\left|\xi-\xi^{\prime}\right|_{\infty}^{\frac{1}{\log q}}
$$

Furthermore, for every $\gamma$ in $\Gamma-\Gamma_{0}$ such that $\gamma \alpha$ and $\gamma \alpha^{*}$ belong to $V$, we have

$$
e^{-D([\gamma])}=c_{*}\left|\gamma \alpha-\gamma \alpha^{*}\right|_{\infty}^{\frac{1}{\log q}}
$$




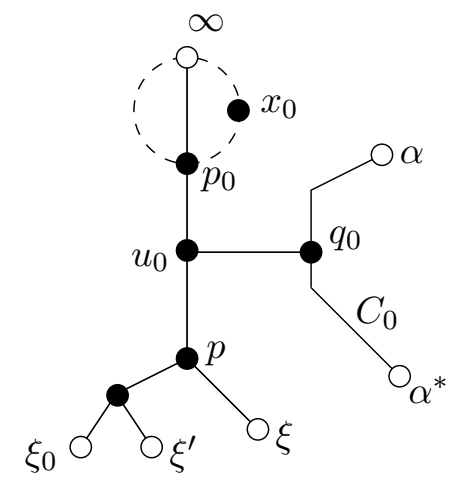

Proof. Let $p_{0}$ be the intersection of the geodesic line $] \infty, \xi_{0}\left[\right.$ in $\mathbb{T}_{q}$ with the horosphere centered at $\infty$ passing through $x_{0}$. Let $q_{0}=\pi_{C_{0}}\left(\xi_{0}\right)$, and $u_{0} \in X$ such that $\left.\left.\left.] \xi_{0}, \infty[\cap] \xi_{0}, q_{0}\right]=\right] \xi_{0}, u_{0}\right]$. In the above picture, we assume that $C_{0}$ and $] \infty, \xi_{0}\left[\right.$ are disjoint, and that $p_{0} \in\left[u_{0}, \infty[\right.$. But the following reasoning is independent of these assumptions. Let $c_{*}=$ $e^{-\beta_{\xi_{0}}\left(q_{0}, p_{0}\right)}$. If $\xi, \xi^{\prime}$ are close enough to $\xi_{0}$, and $] \xi, \infty[\cap] \xi^{\prime}, \infty[=[p, \infty[$, then $p_{0}, u_{0} \in\left[p, \infty\left[, \pi_{C_{0}}(\xi)=\pi_{C_{0}}\left(\xi^{\prime}\right)=q_{0}, \beta_{\xi_{0}}\left(q_{0}, p_{0}\right)=d\left(q_{0}, p\right)-\right.\right.$ $d\left(p_{0}, p\right), d_{\infty}\left(\xi, \xi^{\prime}\right)=e^{-d\left(p_{0}, p\right)}$ and $d_{C_{0}}\left(\xi, \xi^{\prime}\right)=e^{-d\left(q_{0}, p\right)}$, hence the first result follows.

As $d_{C_{0}}\left(\gamma \alpha, \gamma \alpha^{*}\right)=e^{-D([\gamma])}$ if $\gamma \alpha, \gamma \alpha^{*}$ are closed enough to $\xi_{0}$ (see Equation (1)), the second result follows from the first one. q.e.d.

Let $\varphi:[1,+\infty[\rightarrow] 0,1]$ be a map with $t \mapsto f(t)=\varphi\left(q^{t}\right)^{\frac{1}{\log q}}$ slowly varying, and let $g: t \mapsto-\log f(t)=-\log _{q} \varphi\left(q^{t}\right)$, so that $\varphi(t)=$ $q^{-g\left(\log _{q} t\right)}$. By an easy change of variable, the integral $\int_{1}^{+\infty} \varphi(t) / t d t$ diverges if and only if $\int_{1}^{+\infty} f(t)^{\log q} d t$ diverges.

By the above lemma and as $f$ is slowly varying, for every compact subset $A$ of $\widehat{K}-\left\{\alpha, \alpha_{*}\right\}$, there exist positive constants $c_{*}^{\prime}$ (small enough) and $c_{*}^{\prime \prime}$ (big enough) such that for every $\xi$ in $A$,

- if $\left(r_{n}=\left[\gamma_{n}\right]\right)_{n \in \mathbb{N}}$ is a sequence in $R_{0}$ with $D\left(r_{n}\right) \rightarrow+\infty$ as $n \rightarrow$ $+\infty$ and $d_{C_{0}}\left(\xi, \gamma_{n} \alpha\right) \leq c_{*}^{\prime} f\left(D\left(r_{n}\right)\right) e^{-D\left(r_{n}\right)}$ for every $n$ big enough, then $h\left(\gamma_{n} \alpha\right) \rightarrow+\infty$ as $n \rightarrow+\infty$ and, for every $n$ big enough,

$$
\left|\xi-\gamma_{n} \alpha\right|_{\infty}^{\frac{1}{\log q}} \leq e^{-g\left(-\log \left|\gamma_{n} \alpha-\gamma_{n} \alpha^{*}\right|_{\infty}^{\frac{1}{\log q}}\right)}\left|\gamma_{n} \alpha-\gamma_{n} \alpha^{*}\right|_{\infty}^{\frac{1}{\log q}}
$$

that is

$$
\left|\xi-\gamma_{n} \alpha\right|_{\infty} \leq \frac{\varphi\left(h\left(\gamma_{n} \alpha\right)\right)}{h\left(\gamma_{n} \alpha\right)} ;
$$

- conversely, if $\left(\gamma_{n}\right)_{n \in \mathbb{N}}$ is a sequence in $\Gamma$ with $h\left(\gamma_{n} \alpha\right) \rightarrow+\infty$ as $n \rightarrow+\infty$ (in particular $\gamma_{n} \notin \Gamma_{0}$ for $n$ big enough) and $\left|\xi-\gamma_{n} \alpha\right|_{\infty} \leq$ $\frac{\varphi\left(h\left(\gamma_{n} \alpha\right)\right)}{h\left(\gamma_{n} \alpha\right)}$ for every $n$ big enough, then with $r_{n}=\left[\gamma_{n}\right]$, we have $D\left(r_{n}\right) \rightarrow+\infty$ as $n \rightarrow+\infty$ and $d_{C_{0}}\left(\xi, \gamma_{n} \alpha\right) \leq c_{*}^{\prime \prime} f\left(D\left(r_{n}\right)\right) e^{-D\left(r_{n}\right)}$ for every $n$ big enough. 
Hence by Theorem 4.6, if $\int_{1}^{+\infty} \varphi(t) / t d t$ diverges, then for $\mu$-almost every $\xi$ in $\widehat{K}$, there exist a sequence $\left(\beta_{n}\right)_{n \in \mathbb{N}}$ in the congruence class of $\alpha$ in $K_{2}$, with $h\left(\beta_{n}\right) \rightarrow+\infty$ as $n \rightarrow+\infty$, such that $\left|\xi-\beta_{n}\right|_{\infty} \leq \frac{\varphi\left(h\left(\beta_{n}\right)\right)}{h\left(\beta_{n}\right)}$. This can be written as $\liminf \frac{h(\beta)}{\varphi(h(\beta))}|\xi-\beta|_{\infty} \leq 1$, where the lower limit is taken over $\beta$ in the congruence class of $\alpha$ with $h(\beta) \rightarrow+\infty$. Replacing $\varphi$ by $\frac{1}{k} \varphi$ and letting $k$ go to $+\infty$, this proves the divergence part of Theorem 1.6 in the introduction. The convergence part follows similarly.

q.e.d.

By taking $\varphi: t \mapsto t^{-s}$ in Theorem 1.6 with $s \geq 0$, the next result, which in particular says that almost every element of $\widehat{K}$ is badly approximable by quadratic irrational elements of $\widehat{K}$, follows immediatly.

Corollary 6.2. For $\mu$-almost every $x$ in $\widehat{K}, \lim \inf h(\beta)|x-\beta|_{\infty}=0$, and, for every $s>0, \lim h(\beta)^{1+s}|x-\beta|_{\infty}=+\infty$, where the lower limit and limit are taken over the quadratic irrational elements $\beta$ in $\widehat{K}$, in any congruence class, with $h(\beta) \rightarrow+\infty$.

\section{Appendix A. Approximating points by C. S. Aravinda, S. Hersonsky and F. Paulin}

Let $X$ be a proper CAT $(-1)$ geodesic metric space. Let $\Gamma$ be a non elementary discrete group of isometries of $X$, with finite critical exponent $\delta$. In this appendix, we will again apply the geometric avatar of the Borel-Cantelli Lemma, Theorem 3.1, this time to prove a Khintchinetype result for the approximation of a point by geodesic lines in $X$.

Let $x_{0} \in X$ be a base point. For every $C \geq 0$, a point $\xi$ in $\partial_{\infty} X$ will be called a $C$-strongly conical limit point if there exist a geodesic line $\rho$ with $\rho(+\infty)=\xi$ and a sequence $\left(\gamma_{n}\right)_{n \in \mathbb{N}}$ in $\Gamma$, such that $\left(\gamma_{n} x_{0}\right)_{n \in \mathbb{N}}$ converges to $\xi, d\left(\gamma_{n} x_{0}, \rho\right) \leq C$ and $d\left(\gamma_{n} x_{0}, \gamma_{n+1} x_{0}\right) \leq C$. Note that if $\xi$ is a $C$-strongly conical limit point with respect to $x_{0}$, then $\xi$ is a $C^{\prime}$-strongly conical limit point with respect to any other base point $x_{0}^{\prime}$ for $C^{\prime}=C+2 d\left(x_{0}, x_{0}^{\prime}\right)$. And if $\xi$ is a $C$-strongly conical limit point for the geodesic line $\rho$, then $\xi$ is a $(C+\epsilon)$-strongly conical limit point with respect to any other geodesic line $\rho^{\prime}$ asymptotic to $\rho$, for every $\epsilon>0$.

Examples. (1) If $\xi$ is a fixed point of a hyperbolic element $\gamma$ of $\Gamma$, then $\xi$ is a $C$-strongly conical limit point with $C=\max \left\{d\left(x_{0}, A_{\gamma}\right)\right.$, $\left.d\left(x_{0}, \gamma x_{0}\right)\right\}$, where $A_{\gamma}$ is the translation axis of $\gamma$.

(2) If $\Gamma$ is convex-cocompact, then there exists a constant $C \geq 0$ such that any limit point of $\Gamma$ is a $C$-strongly conical limit point.

The following result is (a slight adaptation of) Sullivan's well-known shadow lemma, see for instance [Bou1, page 93].

Lemma A.1. For every conformal density $\left(\mu_{z}\right)_{z \in X}$ of dimension $\delta$ for $\Gamma$, for every $C \geq 0$, there exists $c \geq 1$ such that for every $C$-strongly 
conical limit point $\xi$, for every $\epsilon \in] 0,1]$,

$$
\frac{1}{c} \epsilon^{\delta} \leq \mu_{x_{0}}\left(B_{d_{x_{0}}}(\xi, \epsilon)\right) \leq c \epsilon^{\delta} .
$$

The following result is the main tool from which Theorem A.3 will follow.

Theorem A.2. Let $X$ be a CAT(-1) proper geodesic metric space. Let $x, y$ be points in $X$. Let $\Gamma$ be a non elementary discrete subgroup of isometries of $X$. Let $\left(\mu_{z}\right)_{z \in X}$ be a conformal density of dimension $\delta$ for $\Gamma$, for some $\delta$ in $] 0,+\infty[$. Assume that Card $\{\gamma \in \Gamma: d(x, \gamma y) \leq n\} \asymp$ $e^{\delta n}$, and that there exists $C>0$ such that for all but finitely many $z$ in $\Gamma y$, there exists a geodesic ray $\rho_{z}$ starting from $x$, passing through $z$ and ending at a $C$-strongly conical limit point. Let $f:[0,+\infty[\rightarrow] 0,+\infty[$ be a slowly varying map, with $f(t)$ converging to 0 as $t \rightarrow+\infty$. Let $E_{f}$ be the set of points in $\partial_{\infty} X$ which belong to infinitely many balls $B_{d_{x}}\left(\rho_{\gamma y}(+\infty), f(d(x, \gamma y)) e^{-d(x, \gamma y)}\right)$ for $\gamma$ in $\Gamma$.

$[A]$ If $\int_{1}^{\infty} f(t)^{\delta} d t$ converges, then $\mu_{x}\left(E_{f}\right)=0$.

$[B]$ If there exists $c$ in $\mathbb{R}$ and a sequence $\left(t_{n}\right)_{n \in \mathbb{N}}$ in $\mathbb{R}$, such that $t_{n} \rightarrow+\infty$ as $n \rightarrow+\infty$, such that $t_{n+1} \geq t_{n}-\log f\left(t_{n}\right)-c$ for every $n$, and such that $\sum_{n \in \mathbb{N}} f\left(t_{n}\right)^{\delta}$ diverges, then $\mu_{x}\left(E_{f}\right)>0$.

Proof. We first start by defining the constants that will be used in this proof.

By an easy geometric series argument, and since the stabilizer of $y$ in $\Gamma$ is finite, there exist $N \in \mathbb{N}-\{0\}$ and $c_{0}>0$ such that $N e^{-N} \leq 1$ and, for every $t \geq 0$, if $J_{t}=\{z \in \Gamma y: t \leq d(x, z)<t+N\}$, then

$$
\frac{1}{c_{0}} e^{\delta t} \leq \text { Card } J_{t} \leq c_{0} e^{\delta t} .
$$

Since $f$ is slowly varying, there exists $c_{1} \geq 1$ such that $f(y) \leq c_{1} f(x)$ if $|y-x| \leq N$. Let $c_{2}=\min \left\{N, \frac{1}{c_{1}}\right\} e^{-N}$, which belongs to ] 0,1$]$. Note that the constant $c_{1}$, and hence $c_{2}$, is unchanged if one replaces $f$ by a scalar multiple of it.

Let $t_{0}^{\prime}>0$ be big enough so that $f(t) \leq e^{-N-c}$ for $t \geq t_{0}^{\prime}$, and that for every $z$ in $\Gamma y$ such that $d(x, z) \geq t_{0}^{\prime}$, the geodesic ray $\rho_{z}$ is defined.

We now define the various objects needed to apply Theorem 3.1.

In Case $[A]$, define $t_{0}=t_{0}^{\prime}$ and by induction $t_{n+1}=t_{n}+N$ for every $n$ in $\mathbb{N}$. In Case $[B]$, as $t_{n} \rightarrow+\infty$, we may assume, up to shifting the indices, that $t_{0} \geq t_{0}^{\prime}$.

Recall that, for every $\epsilon>0$, a subset $S$ of a metric space is called $\epsilon$-separated if for every $s \neq s^{\prime}$ in $S$, we have $d\left(s, s^{\prime}\right)>\epsilon$. Endow the (discrete) orbit $\Gamma y$ with the induced metric. For every $n$ in $\mathbb{N}$, let $I_{n}$ be a maximal $4 N$-separated subset of $J_{t_{n}}$ and $I=\bigcup_{n=0}^{\infty} I_{n}$. The subsets $J_{t_{n}}$ for $n$ in $\mathbb{N}$, and hence the $I_{n}$ 's, are pairwise disjoint, in Case [A] by 
the definition of $\left(t_{n}\right)_{n \in \mathbb{N}}$, and in Case $[B]$ since by induction $t_{n} \geq t_{0}^{\prime}$ and $t_{n+1} \geq t_{n}-\log f\left(t_{n}\right)-c \geq t_{n}+N$.

For every $n$ in $\mathbb{N}$ and $\epsilon>0$, define

$$
\begin{gathered}
f_{1}(n)=e^{\delta t_{n}}, \quad f_{2}(n)=c_{2} e^{-t_{n}}, \quad f_{3}(n)=c_{2} f\left(t_{n}\right) e^{-t_{n}}, \\
f_{4}(n)=c_{2}^{-\delta}, \quad f_{5}(\epsilon)=\epsilon^{\delta} .
\end{gathered}
$$

The series $\sum_{n \in \mathbb{N}} f_{1}(n) f_{4}(n) f_{5}\left(f_{3}(n)\right)$ converges if and only if the series $\sum_{n \in \mathbb{N}} f\left(t_{n}\right)^{\delta}$ converges, which in Case $[A]$ is true if and only if the integral $\int_{1}^{+\infty} f^{\delta}$ converges, as $f$ is slowly varying.

For every $z$ in $I$ and $\epsilon>0$, let $B_{z}(\epsilon)=B_{d_{x}}\left(\rho_{z}(+\infty), \epsilon\right)$, which is measurable and non-decreasing in $\epsilon$.

Let us prove that the finite measured space $\left(\partial_{\infty} X, \mu_{x}\right)$, the family $\left(B_{z}(\epsilon)\right)_{z \in I, \epsilon>0}$, the finite-to-one map $I \rightarrow \mathbb{N}$ defined by $z \mapsto n_{z}=n$ if $z \in I_{n}$, and the above maps $f_{1}, f_{2}, f_{3}, f_{4}, f_{5}$ satisfy the assumptions of Theorem 3.1.

Assumption (1) of Theorem 3.1 is satisfied since $f\left(t_{n}\right) \leq 1$ for every $n$. Assumption (2) is satisfied by the definition of $\left(f_{i}\right)_{1 \leq i \leq 5}$. Assumption (3) holds true with (for instance) $c^{\prime}=2, c^{\prime \prime}=2^{\delta}$. Assumption (5) is satisfied by Lemma A.1, since $f_{2} \leq 1$ and $f_{4}$ is constant.

Let us prove that Assumption (4) of Theorem 3.1 holds. By the maximality of $I_{n}$, for every $z^{\prime}$ in $J_{t_{n}}$, there exists $z \in I_{n}$ such that $d\left(z, z^{\prime}\right) \leq 4 N$. Hence

$$
\text { Card } I_{n} \leq \text { Card } J_{t_{n}} \leq(\operatorname{Card} B(y, 4 N) \cap \Gamma y)\left(\operatorname{Card} I_{n}\right) .
$$

By Equation (16), we have Card $I_{n} \asymp e^{\delta t_{n}}$ as wanted.

Let us prove that Assumption (6) of Theorem 3.1 holds. Given $n$ in $\mathbb{N}$ and distinct $z, z^{\prime}$ in $I_{n}$, assume by contradiction that $B_{z}\left(f_{2}(n)\right)$ intersects $B_{z^{\prime}}\left(f_{2}(n)\right)$ non trivially. By Lemma $2.1(1)$, for every $\gamma \in \Gamma$, the ball $B_{d_{x}}\left(\rho_{\gamma y}(+\infty), N e^{-d(x, \gamma y)}\right)$ is contained in $\mathscr{O}_{x} B(\gamma y, N)$. As $d(x, z) \leq t_{n}+N$ and $c_{2} \leq N e^{-N}$, we have $f_{2}(n) \leq N e^{-d(x, z)}$, and similarly for $z^{\prime}$. Therefore $\mathscr{O}_{x} B(z, N)$ intersects $\mathscr{O}_{x} B\left(z^{\prime}, N\right)$ non trivially. Hence there exists a geodesic ray $\rho$ starting from $x$ and passing at distance at most $N$ from both $z$ and $z^{\prime}$. Let $p, p^{\prime}$ be the closest point of $z, z^{\prime}$ respectively on $\rho$, with (up to permuting $z$ and $z^{\prime}$ ) $p^{\prime} \in[x, p]$. As $z, z^{\prime} \in I_{n}$ and since the closest point maps do not increase distances, we have

$$
\begin{aligned}
d\left(z, z^{\prime}\right) & \leq d\left(p, p^{\prime}\right)+2 N=d(x, p)-d\left(x, p^{\prime}\right)+2 N \\
& \leq d(x, z)-d\left(x, z^{\prime}\right)+3 N \leq 4 N .
\end{aligned}
$$

This contradicts the fact that $I_{n}$ is $4 N$-separated.

Finally, let us prove that Assumption (7) of Theorem 3.1 holds under the hypotheses of Case $[B]$. For $m>n$, take $\left(z, z^{\prime}\right)$ in $I_{n} \times I_{m}$, and assume that $B_{z}\left(f_{3}(n)\right)$ intersects $B_{z^{\prime}}\left(f_{3}(m)\right)$ in at least one point $\xi$. 
Since $f_{3} \leq f_{2}, t_{m} \geq t_{n+1}$ and $t_{n+1} \geq t_{n}-\log f\left(t_{n}\right)-c$, we have $f_{3}(m) \leq$ $f_{2}(m) \leq f_{2}(n+1) \leq e^{c} f_{3}(n)$. Then, for every $\eta$ in $B_{z^{\prime}}\left(f_{2}(m)\right)$, we have

$$
\begin{aligned}
d_{x}\left(\eta, \rho_{z}(+\infty)\right) & \leq d_{x}\left(\eta, \rho_{z^{\prime}}(+\infty)\right)+d_{x}\left(\rho_{z^{\prime}}(+\infty), \xi\right)+d_{x}\left(\xi, \rho_{z}(+\infty)\right) \\
& \leq f_{2}(m)+f_{3}(m)+f_{3}(n) \leq\left(2 e^{c}+1\right) f_{3}(n) .
\end{aligned}
$$

Therefore $B_{z^{\prime}}\left(f_{2}(m)\right)$ is contained in $B_{z}\left(\left(2 e^{c}+1\right) f_{3}(n)\right)$, which proves the claim.

Let $E_{f}^{\prime}$ be the subset of points of $\partial_{\infty} X$ which, as $z$ ranges over $I$, belong to infinitely many balls $B_{z}\left(f_{3}\left(n_{z}\right)\right)$. As $0 \leq d(x, z)-t_{n} \leq N$ if $z \in I_{n}$, and since $c_{2} \leq \frac{1}{c_{1}} e^{-N}$, we have, for every $z$ in $I_{n}$,

$$
B_{z}\left(f_{3}(n)\right) \subset B_{d_{x}}\left(\rho_{z}(+\infty), f(d(x, z)) e^{-d(x, z)}\right) \subset B_{z}\left(\frac{c_{1}}{c_{2}} f_{3}(n)\right) .
$$

In Case $[B]$, it follows from Theorem 3.1 that $\mu_{x}\left(E_{f}^{\prime}\right)>0$. By the first inclusion in Equation (17), we have $\mu_{x}\left(E_{f}\right)>0$.

In Case $[A]$, if $g=\frac{c_{1}}{c_{2}} f$, then $\int_{1}^{+\infty} g^{\delta}$ also converges. Hence Theorem 3.1 implies that $\mu_{x}\left(E_{g}^{\prime}\right)=0$. It follows from the second inclusion in Equation (17) that $\mu_{x}\left(E_{f}\right)=0$.

q.e.d.

Given a complete Riemannian manifold $M$ and a map $f:[0,+\infty[\rightarrow$ ]0, $+\infty$ [, say that an element $v$ in $T^{1} M$, or its associated geodesic line $\rho$ in $M$, is $f$-well approximating $x_{0}$ (we said $v$ is $f$-Liouville at $x_{0}$ in [HP1]) if there exists a sequence of times $\left(t_{n}\right)_{n \in \mathbb{N}}$ tending to $+\infty$ such that $d\left(\rho\left(t_{n}\right), x_{0}\right) \leq f\left(t_{n}\right)$ for every $n$.

When $M$ is a finite volume negatively curved locally symmetric space of dimension $n, \mathrm{~F}$. Maucourant [Mau] proved that if $f$ is decreasing to 0 , then for the Liouville measure (which in general strongly differs from the maximal entropy measure), almost every (respectively almost no) $v \in$ $T^{1} M$ is $f$-well approximating $x_{0}$ if and only if $\int_{1}^{\infty} f(t)^{n-1} d t$ diverges (respectively converges). Hence in constant curvature, the following result is weaker than Maucourant's theorem.

Theorem A.3. Let $M$ be a closed manifold with sectional curvature at most -1 , let $x_{0}$ be a point in $M$, and let $\mu$ be the maximal entropy probability measure for the geodesic flow of $M$, with $h$ its topological entropy. Let $f:[0,+\infty[\rightarrow] 0,+\infty[$ be a non increasing and slowly varying map.

(1) If $f(t)$ and $e^{-t} / f(t)$ converge to 0 as $t \rightarrow+\infty$, if the integral $\int_{u}^{\infty} \frac{f(t)^{h}}{-\log f(t)} d t$ diverges (for some $u$ big enough), then $\mu$-almost every geodesic line is $f$-well approximating $x_{0}$.

(2) If the sectional curvature of $M$ satisfies $-a^{2} \leq K \leq-1$, and if $\int_{1}^{\infty} f(t)^{\frac{h}{a}} d t$ converges, then $\mu$-almost no geodesic line is $f$-well approximating $x_{0}$. 
Note that in Case (1), the assumptions that $f(t)$ and $e^{-t} / f(t)$ converge to 0 are not very important. If $f(t)$ is at least a positive constant, then simply by ergodicity, $\mu$-almost every geodesic line is $f$-well approximating $x_{0}$. And if $e^{-t} / f(t)$ is a least a positive constant, then the integral $\int_{u}^{\infty} \frac{f^{h}}{-\log f}$ converges for $u$ big enough.

Proof. Let $X \rightarrow M$ be a universal covering of $M$, with covering group $\Gamma$ (which is non elementary), let $x$ be a lift of $x_{0}$, and take $y=x$. For every $z$ in $\Gamma y-\{y\}$, let $\rho_{z}$ be the geodesic ray starting from $x$ through $z$, which ends at a (uniformly) strongly conical limit point, since $M$ is compact. Let $\left(\mu_{z}\right)_{z \in X}$ be the (unique up to positive scalar multiple) ergodic conformal density of dimension equal to the critical exponent $\delta$ of $\Gamma$ (which is equal to $h$, as $M$ is compact). Since $M$ is compact, we have $\operatorname{Card}\{\gamma \in \Gamma: d(x, \gamma y) \leq n\} \asymp e^{\delta n}$. Hence the general hypotheses of Theorem A.2 on $X, \Gamma,\left(\mu_{z}\right)_{z \in X}$ are satisfied.

Let us prove the first assertion of Theorem A.3. Fix a slowly varying non increasing map $f_{*}:\left[0,+\infty[\rightarrow] 0, \frac{1}{e}\right]$ such that $\frac{f_{*}(t)}{f(t)}$ tends to 0 as $t \rightarrow+\infty$ (so that in particular $f_{*}$ converges to 0 at $+\infty$ ) and the integral $\int_{u}^{\infty} \frac{f_{*}(t)^{h}}{-\log f_{*}(t)} d t$ still diverges. Let $t_{0}=1$. Define by induction $t_{n+1}=t_{n}-\log f_{*}\left(t_{n}\right)$. In particular, the sequence $\left(t_{n}\right)_{n \in \mathbb{N}}$ converges to $+\infty$. As $f_{*}$ is non increasing, we have

$$
\int_{t_{n}}^{t_{n+1}} \frac{f_{*}(t)^{\delta}}{-\log f_{*}(t)} d t \leq \frac{f_{*}\left(t_{n}\right)^{\delta}}{-\log f_{*}\left(t_{n}\right)}\left(t_{n+1}-t_{n}\right)=f_{*}\left(t_{n}\right)^{\delta} .
$$

Hence $\sum_{n \in \mathbb{N}} f_{*}\left(t_{n}\right)^{\delta}$ diverges. Therefore the hypotheses of Theorem A.2 [B] are satisfied for $f_{*}$.

Denote by $\mathscr{S}$ the measurable set of elements $\xi$ in $\partial_{\infty} X$ which belong to infinitely many visual balls $B_{d_{x}}\left(\rho_{\gamma x}(+\infty), f_{*}(d(x, \gamma x)) e^{-d(x, \gamma x)}\right)$ as $\gamma$ ranges over $\Gamma$. By Lemma 2.1 (1), this ball is contained in the shadow $\mathscr{O}_{x}\left(B\left(\gamma x, f_{*}(d(x, \gamma x))\right)\right)$. For every $\xi$ in $\mathscr{S}$, let $\rho_{\xi}$ be the geodesic ray starting from $x$ and ending at $\xi$. Since $f_{*}$ is slowly varying, there exists $c_{1}>0$ such that $f_{*}(t)<c_{1} f_{*}(s)$ if $|t-s| \leq 1$. As $f_{*}(t) \leq 1$, there exist a sequence $\left(s_{n}\right)_{n \in \mathbb{N}}$ in $[0,+\infty$ [ converging to $+\infty$ and a sequence $\left(\gamma_{n}\right)_{n \in \mathbb{N}}$ in $\Gamma$ such that $d\left(\rho_{\xi}\left(s_{n}\right), \gamma_{n} x\right) \leq c_{1} f_{*}\left(s_{n}\right)$ for every $n$. For every $\xi$ in $\mathscr{S}$, let $\mathscr{S}_{\xi}$ be the set of elements $v$ in $T^{1} M$ such that the point at infinity of the geodesic line $\rho_{v}$, which is some lift by $X \rightarrow M$ of the geodesic line in $M$ associated to $v$, is equal to $\xi$. Since $X$ is $\operatorname{CAT}(-1)$, if $\rho, \rho^{\prime}$ are asymptotic geodesic rays, then there exists $c>0$ and $\tau \in \mathbb{R}$ such that $d\left(\rho(t), \rho^{\prime}(t+\tau)\right) \leq c e^{-t}$ for every $t \geq \max \{0,-\tau\}$. Since $f_{*}$ is slowly varying, for every $v \in \mathscr{S}_{\xi}$, there exist hence a constant $c^{\prime}>0$ and sequences $\left(s_{n}^{\prime}\right)_{n \in \mathbb{N}}$ in $\left[0,+\infty\right.$ [ converging to $+\infty$ and $\left(\gamma_{n}\right)_{n \in \mathbb{N}}$ in $\Gamma$ such that

$$
d\left(\rho_{v}\left(s_{n}^{\prime}\right), \gamma_{n} x\right) \leq c^{\prime}\left(f_{*}\left(s_{n}^{\prime}\right)+e^{-s_{n}^{\prime}}\right) .
$$


The right hand side is at most $f\left(s_{n}^{\prime}\right)$ for $n$ big enough, since $\frac{f_{*}(t)}{f(t)}$ and $e^{-t} / f(t)$ tend to 0 as $t \rightarrow+\infty$. Hence every $v \in \mathscr{S}_{\xi}$ is $f$-well approximating $x_{0}$, for every $\xi \in \mathscr{S}$. By Theorem A.2 [B], the set $\mathscr{S}$ has positive measure for $\mu_{x}$. Hence the set $\mathscr{S}^{\prime}=\bigcup_{\xi \in \mathscr{S}} \mathscr{S}_{\xi}$ (which is measurable, as the direct image by the covering map $T^{1} X \rightarrow T^{1} M$ of the preimage by the continuous endpoint map $T^{1} X \rightarrow \partial_{\infty} X$ of $\mathscr{S}$ ) has positive measure for $\mu$, by Lemma 2.4. As $\mathscr{S}^{\prime}$ is invariant under the geodesic flow by construction, and by ergodicity, it has full measure. This proves the result.

Let us now prove the assertion (2) of Theorem A.3. As $f$ is slowly varying, the convergence of the integral $\int_{1}^{\infty} f^{\frac{\delta}{a}}$ implies that $f$ converges to 0 as $t \rightarrow+\infty$. For every $v$ in $T^{1} M$, let $\rho_{v}$ be a lift by $X \rightarrow M$ of the geodesic line in $M$ associated to $v$, let $\xi_{v}=\rho_{v}(+\infty)$ and let $\rho_{v}^{\prime}$ be the geodesic ray from $x$ to $\xi_{v}$. If $v$ is $f$-well approximating $x_{0}$, then there exist a sequence $\left(s_{n}\right)_{n \in \mathbb{N}}$ in $[0,+\infty$ [ converging to $+\infty$ and a sequence $\left(\gamma_{n}\right)_{n \in \mathbb{N}}$ in $\Gamma$ such that $d\left(\rho_{v}\left(s_{n}\right), \gamma_{n} x\right) \leq f\left(s_{n}\right)$ for every $n$. Since $f$ is slowly varying and since $\rho_{v}^{\prime}$ and $\rho_{v}$ are asymptotic, as above, there exist $k$ in $\mathbb{N}-\{0\}$, a sequence $\left(s_{n}^{\prime}\right)_{n \in \mathbb{N}}$ in $[0,+\infty$ [ converging to $+\infty$, and a sequence $\left(\gamma_{n}\right)_{n \in \mathbb{N}}$ in $\Gamma$, such that $d\left(\rho_{v}^{\prime}\left(s_{n}^{\prime}\right), \gamma_{n} x\right) \leq k\left(f\left(s_{n}^{\prime}\right)+e^{-s_{n}^{\prime}}\right)$ for every $n$. In particular, as $f(t) \leq 1$ if $t$ is big enough, if $v$ is $f$-well approximating $x_{0}$, then there exists $k^{\prime}$ in $\mathbb{N}-\{0\}$ such that $\xi_{v}$ belongs to infinitely many shadows $\mathscr{O}_{x}\left(B\left(\gamma x, k^{\prime}\left(f(d(x, \gamma x))+e^{-d(x, \gamma x)}\right)\right)\right)$ as $\gamma$ ranges over $\Gamma$. By Lemma 2.1 (2), this shadow is contained, except for finitely many $\gamma \in \Gamma$, in the ball

$$
\mathscr{B}_{\gamma, k^{\prime \prime}}=B_{d_{x}}\left(\rho_{\gamma x}(+\infty), k^{\prime \prime}\left(f(d(x, \gamma x))+e^{-d(x, \gamma x)}\right)^{\frac{1}{a}} e^{-d(x, \gamma x)}\right),
$$

for some positive integer $k^{\prime \prime}$. If $u, v, w>0$, recall that $(u+v)^{w} \leq 2^{w}\left(u^{w}+\right.$ $\left.v^{w}\right)$. Hence since $\int_{1}^{\infty} f^{\frac{\delta}{a}}$ converges, the integral $\int_{1}^{\infty}\left(k^{\prime \prime}\left(f(t)+e^{-t}\right)^{\frac{1}{a}}\right)^{\delta} d t$ also converges. The map $t \mapsto k^{\prime \prime}\left(f(t)+e^{-t}\right)$ is slowly varying. By Theorem A.2 $[A]$, the measure of the set of points in $\partial_{\infty} X$ which belong to infinitely many balls $\mathscr{B}_{\gamma, k^{\prime \prime}}$, as $\gamma$ ranges over $\Gamma$, has measure 0 for $\mu_{x}$. By Lemma 2.4, and since a countable union of measure zero subsets is a measure zero subset, the result follows.

q.e.d.

For every $\alpha>0$, let $f_{\alpha}: t \mapsto \frac{1}{(2+t)^{\alpha}}$, which is slowly varying, with $f(t)$ and $e^{-t} / f(t)$ converging to 0 as $t \rightarrow+\infty$. For every $h>0$, the integral $\int_{1}^{\infty} \frac{f_{\alpha}^{h}}{-\log f_{\alpha}}$ diverges if and only if $\alpha \leq \frac{1}{h}$ and the integral $\int_{1}^{\infty} f_{\alpha}^{h}$ converges if and only if $\alpha>\frac{1}{h}$. By applying Theorem A.3 with $M$ having constant curvature -1 , so that $h=n-1$, with $f=f_{\frac{1}{h} \pm \frac{1}{n}}$ where $n \rightarrow+\infty$, Theorem 1.7 of the introduction follows, in the standard way one deduces a logarithm law-type theorem from a Khintchine-type theorem. 


\section{References}

[Arz] G. Arzhantseva, On quasi-convex subgroups of word hyperbolic groups, Geom. Dedicata 87 (2001) 191-208, MR 1866849, Zbl 0994.20036.

[Bea] A. Beardon, The geometry of discrete groups, Springer-Verlag, 1983, MR 1393195, Zbl 0528.30001.

[BV] V. Beresnevich \& S. Velani, Ubiquity and a general logarithm law for geodesics, preprint Univ. York, July 2007, to appear in the proceedings of "Dynamical systems and Diophantine Approximation", Institut Henri Poincaré, 7-9 June 2004. [arXiv:0707.1225]

[Bou] N. Bourbaki, Topologie générale, chap. 1 à 4, Hermann, Paris, 1971, MR 0358652, Zbl 0249.54001.

[Bou1] M. Bourdon, Structure conforme au bord et flot géodésique d'un CAT(-1) espace, L'Ens. Math. 41 (1995) 63-102, MR 1341941, Zbl 0871.58069.

[Bou2] M. Bourdon, Immeubles hyperboliques, dimension conforme et rigidité de Mostow, GAFA 7 (1997) 245-268, MR 1445387, Zbl 0876.53020.

$[\mathrm{BH}] \quad$ M. R. Bridson \& A. Haefliger, Metric spaces with non-positive curvature, Grund. math. Wiss. 319, Springer Verlag, 1998, MR 1744486, Zbl 0988.53001.

[BP] A. Broise \& F. Paulin, Dynamique sur le rayon modulaire et fractions continues en caractéristique p, J. London Math. Soc. 76 (2007) 399-418, MR 2363423, Zbl 1133.11043.

[DMPV] M. M. Dodson, M. V. Meliàn, D. Pestana \& S. L. Velani, Patterson measure and ubiquity, Ann. Acad. Sci. Fenn. 20 (1995) 37-60, MR 1304105, Zbl 0816.11043.

[Fur] H. Furusawa, The exponent of convergence of Poincaré series of combination groups, Tohoku Math. J. 43 (1991) 1-7, MR 1088710, Zbl 0739.20007.

[GP] D. Gaboriau \& F. Paulin, Sur les immeubles hyperboliques, Geom. Dedi. 88 (2001) 153-197, MR 1877215, Zbl 1020.51007.

[GH] E. Ghys \& P. de la Harpe, eds. Sur les groupes hyperboliques d'après Mikhael Gromov, Prog. in Math. 83, Birkhäuser, 1990, MR 1086648, Zbl 0731.20025.

[Haa1] A. Haas, The distribution of geodesic excursions out the end of a hyperbolic orbifold and approximation with respect to a Fuchsian group, Geom. Dedicata 116 (2005) 129-155, MR 2195445, Zbl 1090.53069.

[Haa2] A. Haas, Geodesic cusp excursions and metric Diophantine approximation, Math. Res. Let. 16 (2009) 67-85, MR 2480562, Zbl 1178.30055.

[HaP] F. Haglund \& F. Paulin, Constructions arborescentes d'immeubles, Math. Ann. 325 (2003) 137-164, MR 1957268, Zbl 1025.51014.

[Ham] U. Hamenstädt, A new description of the Bowen-Margulis measure, Erg. Theo. Dyn. Sys. 9 (1989) 455-464, MR 1016663, Zbl 0722.58029.

[HP1] S. Hersonsky \& F. Paulin, On the rigidity of discrete isometry groups of negatively curved spaces, Comm. Math. Helv. 72 (1997) 349-388, MR 1476054, Zbl 0908.57009.

[HP2] S. Hersonsky \& F. Paulin, Hausdorff dimension of diophantine geodesics in negatively curved manifolds, J. reine angew. Math. 539 (2001) 29-43, MR 1863852, Zbl 0994.53021.

[HP3] S. Hersonsky \& F. Paulin, Diophantine approximation for negatively curved manifolds, I, Math. Zeit. 241 (2002) 181-226, MR 1930990, Zbl 1037.53020. 
[HP4] S. Hersonsky \& F. Paulin, Counting orbit points in coverings of negatively curved manifolds and Hausdorff dimension of cusp excursions, Erg. Theo. Dyn. Sys. 24 (2004) 1-22, MR 2060999, Zbl 1059.37022.

[HP5] S. Hersonsky \& F. Paulin, A logarithm law for automorphism groups of trees, Archiv Math. 88 (2007) 97-108, MR 2299033, Zbl 1160.20020.

[HS] M. Hindry \& J. Silverman, Diophantine geometry : an introduction, GTM 201, Springer Verlag, 2000, MR 1745599, Zbl 0948.11023.

[Kai] V. A. Kaimanovich, Bowen-Margulis and Patterson measures on negatively curved compact manifolds, in "Dynamical systems and related topics" (Nagoya, 1990), 223-232, Adv. Ser. Dyn. Syst., 9, World Sci. Pub., 1991, MR 1164891.

[Kap] M. Kapovich, Representations of polygons of finite groups, Geom. Topol. 9 (2005) 1915-1951, MR 2175160, Zbl 1163.20029.

[KS] I. Kapovich \& H. Short, Greenberg's theorem for quasiconvex subgroups of word hyperbolic groups, Canadian J. Math., 48 (1996) 1224-1244, MR 1426902, Zbl 0873.20025.

[KM] D. Kleinbock \& G. Margulis, Flows on homogeneous spaces and Diophantine approximation on manifolds, Ann. Math. 148 (1998) 339-360, MR 1652916, Zbl 0922.11061.

[Kni] G. Knieper, Spherical means on compact Riemannian manifolds of negative curvature, Diff. Geom. App. 4 (1994) 361-390, MR 1306567, Zbl 0812.53043.

[Las] A. Lasjaunias, A survey of Diophantine approximation in fields of power series, Monat. Math. 130 (2000) 211-229, MR 1777096, Zbl 0990.11043.

[Leu] E. Leuzinger, Kazhdan's property (T), $L^{2}$-spectrum and isoperimetric inequalities for locally symmetric spaces, Comment. Math. Helv. 78 (2003) 116-133, MR 1966754, Zbl 1027.22015.

[Man] A. Manning, Topological entropy for geodesic flows, Ann. Math. 110 (1979) 567-573, MR 0554385, Zbl 0426.58016.

[Mau] F. Maucourant, Dynamical Borel-Cantelli lemma for hyperbolic spaces, Israel J. Math. 152 (2006) 143-155, MR 2214457, Zbl 1129.53057.

[PP1] J. Parkonnen \& F. Paulin, Prescribing the behaviour of geodesics in negative curvature, Geom. \& Topo. 14 (2010) 277-392, MR 2578306, Zbl pre05624192.

[PP2] J. Parkonnen \& F. Paulin, Spiraling spectra of geodesics in negative curvature, Preprint Univ. Jyvaskyla, june 2008, to appear in Mathematische Zeitschrift. [arXiv:0806.1857]

[Pau] F. Paulin, Groupe modulaire, fractions continues et approximation diophantienne en caractéristique p, Geom. Dedicata 95 (2002) 65-85, MR 1950885, Zbl 1033.20026.

[Rob] T. Roblin, Ergodicité et équidistribution en courbure négative, Mémoires Soc. Math. France, 95 (2003), MR 2057305, Zbl 1056.37034.

[Sch] W. Schmidt, On continued fractions and Diophantine approximation in power series fields, Acta Arith. XCV (2000) 139-166, MR 1785412, Zbl 0987.11041.

[Ser] J.-P. Serre, Arbres, amalgames, $S L_{2}$, Astérisque 46, Soc. Math. France, 1983, MR 0476875, Zbl 0369.20013. 
[Spr] V. G. Sprindžuk, Mahler's problem in metric number theory, Transl. Math. Mono 25, Amer. Math. Soc., 1969, MR 0245527, Zbl 0181.05502.

[SV] B. Stratmann \& S. L. Velani, The Patterson measure for geometrically finite groups with parabolic elements, new and old, Proc. London Math. Soc. 71 (1995) 197-220, MR 1327939, Zbl 0821.58026.

[Sul] D. Sullivan, Disjoint spheres, approximation by imaginary quadratic numbers, and the logarithm law for geodesics, Acta Math. 149 (1982) 215-237, MR 0688349, Zbl 0517.58028.

[SS] P. Susskind \& G. A. Swarup, Limit sets of geometrically finite hyperbolic groups, Amer. J. Math. 114 (1992) 233-250, MR 1156565, Zbl 0791.30039.

UNIVERSity OF GEORGIA Department of Mathematics Athens, GA 30602

E-mail address: saarh@math.uga.edu

Département de Mathématique et Applications UMR 8553 CNRS

École Normale Supérieure, 45 RUe D'Ulm 75230 Paris Cedex 05, France E-mail address: Frederic.Paulin@ens.fr 